\title{
Recherches analytiques sur un cas de rotation d'un solide pesant autour d'un point fixe.
}

Par

Mr. P. A. Nekrassoff à Moscou.

J'ai en vue de présenter dans cet article l'exposition des propriétés du mouvement d'un solide pesant autour d'un point fixe. Je n'expose principalement que mes recherches personnelles en eitant, aux places convenantes, les travaux des auteurs qui en parlent, car depuis peu il a paru beaucoup d'articles concernant ce cas de rotation*), qui, entre autre, est caractérisé par le mouvement du centre de gravité d'après les règles $d u$ pendule sphérique.

Ce cas de rotation d'un solide autour d'un point fixe a été examiné déjà par Mr. W. Hess dans les Mathematische Annalen (volume 37,

*) Nous citons ici le tableau de ces articles russes.

Mr. P. A. Nekrassoff: 1) ,Au sujet du problème de la rotation d'un solide pesant autour d'un point fixe". (Recueil de mathématiques, t. XVI, 3ème livraison, 1892, Moscou).

2) "De la rotation d'un solide pesant autour d'un point fixe" (Mémoires de la section physique de la société Impériale des Amis des sciences naturelles, t. V, 1893, Moscon).

3) "Complément de l'article concernant la rotation d'un solide pesant autour d'un point fixe". (Mémoires de la section physique de la société Impériale des Amis des sciences naturelles, t. VI, 1893, Moscou).

4) „Recherches analytiques sur un cas de rotation d'un solide pesant antour d'un point fixe". (Recueil de mathématiques, t. XVIII, 1895, Moscou).

Mr. N. E. Joukorsky: "Pendule loxodromique de Mr. Hess" (Mémoires de la section physique de la société Impériale des Amis des sciences naturelles, t. V, 1893, Moscou). Cet article est imprimé encore dans „Jahresbericht der dentschen Mathematiker-Vereinigung" (III. 1893, p. 62-70).

Mr. B. C. Mlodzeievaky et Mr. P. A. Nekrassoff: "Des conditions de l'existence des mouvements asymptotiques périodiques dans le problème de Hess" (Mémoires de la section physique de la société impériale des Amis des sciences naturelles, t. VI, 1893, Moscou).

Mr. J. A. Tchapliguin: "Au sujet du pendule loxodromique de Mr. Hess" (Mémoires de la section physiquo de la société Impériale des Axnis des sciences naturelles, t. VII, 1894, Moscou). 
page 178-180). Dans l'ouvrage de Mr. Hess beaucoup de propriétés de ce mouvement sont restées inexpliquées; principalement celles qui dépendent des particularités de l'équation plus compliquée de ce problème. Je suis parvenu à réduire l'intégration de cette dernière à celle de l'équation linéaire du deuxième ordre à coefficients uniformes doublement périodiques complexes. Par ce moyen le problème s'éclaircit à un tel point qu'il devient possible de se représenter assez complètement lo tableau de la rotation et à réduire les calculs aux séries toujours convergentes et qui ont la simplicité permise par le problème. Ainsi on peut placer ce cas de rotation au nombre de ceux qui ont été examinés avec une attention plus profonde.

$\mathrm{Au}$ point de vue purement matbématique le cas de rotation que j'examine présente des particularités différentes. C'est à ees particularités qu'il faut attribuer l'avantage de l'emploi étendu des quantités complexes, dont les qualités géometriques se montrent très utiles dans l'exploration de ce cas, surtout combiné avec l'ainsi nommée transformation linécire qui réduit les lignes droites et les cercles en cercles en conservant la similitude des parties infiniment petites. L'autre particularité du cas considéré consiste en ce que le problème est réduit ici non pas aux fonctions de temps uniformes comme dans les cas du mouvement examinés par Enler, Lagrange et Kovalevsky, mais aux fonctions multiformes.

\section{$\S 1$.}

Equations fondamentales; leurs intégrales algébriques.

En observant les conditions:

$$
y_{0}=0, A(B-C) x_{0}^{2}=C(A-B) z_{0}^{2}, A>B>C,
$$

les équations du mouvement d'un solide pesant autour du point fixe $O$, amenées à la forme*):

$$
\left\{\begin{array}{l}
A \frac{d p}{d t}=(B-C) q r+y_{0} \gamma^{\prime \prime}-z_{0} \gamma^{\prime}, \frac{d \gamma}{d t}=r \gamma^{\prime}-q \gamma^{\prime \prime} \\
B \frac{d q}{d t}=(C-A) r p+z_{0} \gamma-x_{0} \gamma^{\prime \prime}, \frac{d \gamma^{\prime}}{d t}=p \gamma^{\prime \prime}-r \gamma \\
C \frac{d r}{d t}=(A-B) p q+x_{0} \gamma^{\prime}-y_{0} \gamma, \frac{d \gamma^{\prime \prime}}{d t}=q \gamma-p \gamma^{\prime}
\end{array}\right.
$$

$\left.{ }^{*}\right) A, B$ et $C$ sont les moments d'inertie par rapport aux axes principanx de l'inertie, tracés par le point fixe et pris comme axes des coordonnés $x, y$ et $z$ dans lo solide; $x_{0}, y_{0}, z_{0}$ sont les coordonnés dn centre $G$ de gravité; $p, q, r$ sont les projections de la vitesse de rotation autour d'un axe momentané sur les axes déja nommés des coordonnées; $\gamma, \gamma^{\prime}, \gamma^{\prime \prime}$ sont les cosinus des angles de ces axes arec la direction de la gravité. En plus on suppose que la masse $M$ da solide et l'accélération $g$ satisfont à la condition $M g=1$, ce qui, comme on le sait, ne limite que le choix des unités. 
admettent, outre les trois intégrales algébriques:

$$
\left\{\begin{array}{l}
\gamma^{2}+\gamma^{\prime 2}+\gamma^{\prime \prime 2}=l_{0}=1, \\
A p \gamma+B q \gamma^{\prime}+C r \gamma^{\prime \prime}=l_{1}, \\
A p^{2}+B q^{2}+C \gamma^{2}-2\left(x_{0} \gamma+y_{0} \gamma^{\prime}+z_{0} \gamma^{\prime \prime}\right)=l_{2},
\end{array}\right.
$$

encore une quatrième intégrale de $\mathrm{Mr}$. $\mathrm{Hess}_{\text {, }}$ algébrique particulière

$$
A x_{0} p+C z_{0} r=0 \text {. }
$$

Avant de développer les conséquences auxquelles conduit la considération de l'intégrale particulière (4), fixons notre attention paur un instant sur une qualité remarquable des intégrales générales, qui est intéressante, entre autre, par ce qu'elle joint les conditions (1) et l'intégrale particulière (4) avec la méthode de Mme. Kovalevsky, exposée dans le $\S 1$ de son mémoire, concernant le mouvement d'un solide pesant autour d'un point fixe (Acta Mathematica, XII).

Si nous approfondissons arec Mme, Kovalevsky la recherche de tous les cas où les équations différentielles du problème admettent pour les fonctions $p, q, r, \gamma, \gamma^{\prime}$ et $\gamma^{\prime \prime}$, qu'elles déterminent, des pôles, comme des points singuliers, arec la condition, que les coefficients des developpements de ces fonctions en séries dans le domaine des pôles dépendent de cinq constantes arbitraires; il se trouvera parmi ces cas celui qui est caractérisé par les conditions (1). Ainsi, en exécutant les conditions (1) on peut satisfaire les équations (2) par des séries de la forme:

$$
\left\{\begin{array}{l}
p=\sum_{n=0}^{\infty} p_{n} t^{n-1}, \quad \gamma=\sum_{n=0}^{\infty} f_{n} t^{n-2}, \\
q=\sum_{n=0}^{\infty} q_{n} t^{n-1}, \quad \gamma^{\prime}=\sum_{n=0}^{\infty} g_{n} t^{n-2}, \\
r=\sum_{n=0}^{\infty} r_{n} t^{n-1}, \quad \gamma^{\prime \prime}=\sum_{n=0}^{\infty} h_{n} t^{n-2},
\end{array}\right.
$$

qui convergent dans un domaine de zéro et dont les coefficients contiennent cinq constantes arbitraires.

Il s'ensuit que le cas de Mr. Hess aurait pu être trouvé par les procédés de Kovalevsky, puisque ce cas est conteuu dans ces équations et n'a été oublié par elle que par inadvertance*).

Renvoyant les lecteurs qui s'intéressent à cette application de la méthode de Kovalevsky aux articles de Mr. H. Appelroth**), qui

*) C'est justement par ce procédé que j’ai trouvé le cas de Mr. Hess et ce ne fut que plus tard que j'appris l'existence de son mémoire.

**) Mr. H. H. A p pelroth: 1) „Par rapport au premier paragraphe da mémoire 
renferment la démonstration de la propriété déjà nommée des intégrales générales du système (2) arec les conditions (1), je me bornerai à développer dans ce qui suit surtout le cas correspondant à l'intégrale particulière (4).

Malgré le caractère particulier de ce cas, le mouvement qui y correspond ne présente pas un degré de généralité moindre que dans les cas examinés par Euler, Lagrange et Kovalersky. Effectivement en partant de l'intégrale particulière (4) nous perdons une constante arbitraire, mais à sa place nous gagnons une constante dans les quantités $x_{0}, y_{0}, z_{0}, A, B$ et $C$, qui, se soumettant aux conditions (1), sont moins limitées que dans les cas de Euler, Lagrange et Kovalersky.

Quant à la réalisation du cas que j'examine du mourement d'un solide, il faut remarquer avant tout la possibilité de la construction du solide pour lequel les conditions (1) suffisent. Si l'on cherche dans le premier solide donné les points pour lesquels les moments principaux $A, B$ et $C$ et les coordonnées $x_{0}, y_{0}, z_{0}$ du centre $G$ de gravité remplissent les conditions (1), nous trouverons une quantité innombrable de points remplissant la place géométrique représentée par deux lignes droites qui passent par le centre de gravité. Ces lignes droites sont perpendiculaires aux sections circulaires d'un ellipsoïde construit pour le centre de gravité. Cet ellipsoïde, rapporté aux axes principaux $x^{\prime}, y^{\prime}, z^{\prime}$ de l'inertie, est déterminé par l'équation:

où

$$
\frac{x^{\prime 2}}{a^{\prime 2}}+\frac{y^{2}}{b^{2}}+\frac{z^{\prime 2}}{c^{\prime 2}}=1
$$

$$
a^{\prime 2}=\frac{A^{\prime}}{M}, \quad b^{\prime 2}=\frac{B^{\prime}}{M}, \quad e^{\prime 2}=\frac{C^{\prime}}{M},
$$

$A^{\prime}, B^{\prime}, C^{\prime}$ sont des moments d'inertie correspondant à ces axes. Cette conclusion a été faite par Mr. Tschapliguin. Il suffit de fixer l'un des points de deux droites nommées pour avoir un solide capable de reproduire le mouvement que j'examine. En appropriant ensuite ce corps de façon à pouvoir y exciter un mourement dont les circonstances initiales correspondent à l'intégrale particulière (4), il faut avoir en vue que l'axe instantané doit se trouver pendant tout le temps de ce mouvement sur le même plan, déterminé par léquation: $A x_{0} x+C z_{0} z=0$. Si par conséquent il fallait donner au solide la forme d'un gyroscope, e'est justement dans ce plan que devrait être placé l'axe du gyroscope auquel on attache le fil pour le faire tourner.

Voici encore un moyen de réaliser le même mouvement du solide

de Sophie Kovalersky" (Recueil de mathématiques, t. XVI, livxaison 3éme, 1892, Moscou). - 2) "Problème concernant la rotation d'un solide pesant autour d'un point fixe" Mémoires scientifiques de l'Université Impériale de Moscon, section physico-mathématique, liv. 11, 1894). 
examiné pour lequel l'intégrale (4) a de la valeur. Il faut ôter lo solide donné de la position d'équilibre et le livrer à lui-même sans lui donner aucune vitesse initiale, de sorte qu'au commencement du mouvement $p=q=r=0$. Avec ces conditions l'intégrale (4) se réalisera absolument. En même temps dans le deuxième des intégrales (3) la constante $l_{1}$ devient zéro, et la constante $l_{2}$ doit contenter l'inégalité $l_{2}<2 \varrho_{0}$, comme nous nous en convainquons au moyen de la troisième des expressions (3). Par conséquent ce moyen, qui est l'un des plus simples de ceux, qui réalisent le mouvement, appartenant au cas que j'examine, n'embrasse pas ce cas dans toute son étendue.

\section{$\S 2$.}

Réduction da problème à la recherche du mouvement de deux points remarquables.

Supposons que les conditions (1) ont lieu et que les cironstances initiales réalisent en mème temps un mouvement pareil autour du point $O$, auquel correspond l'intégrale particulière (4).

Introduisons les désignations suivantes:

$$
\left\{\begin{array}{c}
\xi=\frac{z_{0}}{\varphi_{0}} \eta-\frac{x_{0}}{\rho_{0}} \gamma^{\prime \prime}, \quad \eta=\gamma^{\prime}, \quad \xi=\frac{x_{0}}{\varphi_{0}} \eta+\frac{z_{0}}{\rho_{0}} \gamma^{\prime \prime}, \\
v=\xi+\eta i, \\
\varrho_{0}=\sqrt{x_{0}{ }^{2}+\underline{\eta}_{0}^{2}}, \quad i=\sqrt{-1} .
\end{array}\right.
$$

Il est évident que $\eta, \xi$ et $\xi$ sont les cosinus des angles de la direction $O V$ de la gravité avec les trois lignes suivantes (réciproquement perpendiculaires: $;$ ) l'axe $y$ de l'inertie, auquel correspond le moment $B$ et qui est représenté sur la figure 1 par la ligne $O \eta$, 2) la ligne $O \zeta$, qui passe par le centre de gravité $G$, et 3) a la ligne $O \xi$, perpendiculaire au plan $\eta O \xi$. D'ailleurs la quantité complexe $v=\xi+\eta i$, qui joue dans le problème un rôle principal, est représentée sur le plan $\eta O \xi$ par le point $v$ (figure 1). Ce point coïncide arec la projection

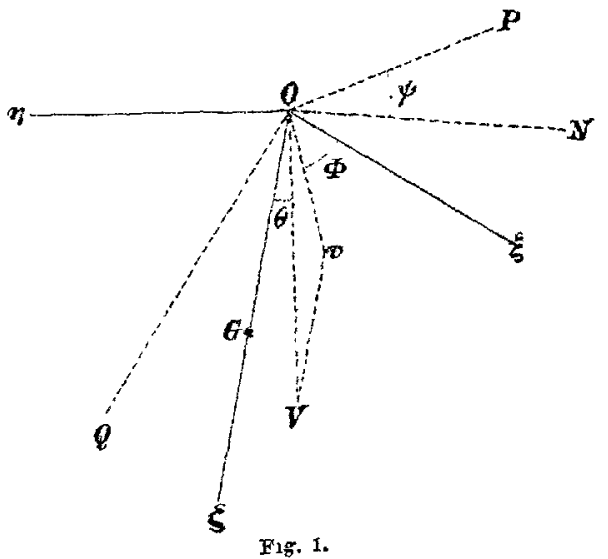
sur le plan du point $V$, qui sépare daus la direction de la gravité e segment $O V$ égal à 1. 
Si nous représentons la sphère $O$, décrite du centre $O$ par le rayon 1 , et si nous prenons le plan $\xi O \eta$ pour le plan de son équateur, l'amplitude $\Phi$ de la quantité $v$, représentée par l'angle $v O \xi$, sera la longitude du point $V$, et l'angle $\theta=\nabla 0 G$, dont le cosinus est $\xi$, sera le complément de la latitude du point $V$ jusqu'à l'angle droit.

Après avoir éliminé des équations (2), (4) et (6) les fonctions $r$, $\gamma, \gamma^{\prime}$ et $\gamma^{\prime \prime}$, nous trouverons les équations différentielles suivantes:

$$
\left\{\begin{aligned}
A \frac{d p}{d t} & =\frac{A(C-B) x_{0}}{C z_{0}} p q-z_{0} \eta \\
B \frac{d q}{d t} & =\frac{A(A-C) x_{0}}{C z_{0}} p^{2}+\varrho_{0} \xi \\
\frac{d \xi}{d t} & =\frac{(C-A) x_{0}}{C \varrho_{0}} p \eta-q \xi \\
\frac{d \eta}{d t} & =\frac{A \varrho_{0}}{B z_{0}} p \zeta-\frac{(C-A) x_{0}}{C_{\varrho_{0}}} p \xi \\
\frac{d \zeta}{d t} & =q \xi-\frac{A \varrho_{0}}{B z_{0}} p \eta .
\end{aligned}\right.
$$

Les trois intégrales connues de ces équations, obtenues par les équations (3), sont:

$$
\left\{\begin{array}{l}
\xi^{2}+\eta^{2}+\xi^{2}=l_{0}=1, \\
\frac{A \varrho_{0}}{z_{0}} p \xi+B q \eta=l_{1}, \\
\frac{A^{2} \varrho_{0}^{2}}{B z_{0}^{2}} p^{2}+B q^{2}-2 \varrho_{0} \xi=l_{2} .
\end{array}\right.
$$

Par ces équations nous trouvons:

(9) $\left\{\begin{array}{c}q=\frac{l_{1} \eta+\xi R}{B\left(1-\xi^{2}\right)}, \quad p=\frac{z_{0}\left(l_{1} \xi-\eta R\right)}{A \varrho_{0}\left(1-\xi^{2}\right)} \\ \eta= \pm \sqrt{1-\xi^{2}-\xi^{2}}, \quad R= \pm \sqrt{B\left(1-\xi^{2}\right)\left(l_{2}+2 \varrho_{0} \xi\right)-l_{1}^{2}} .\end{array}\right.$

Après avoir introduit les quantités $p, q$ et $\eta$ dans la dernière des équations (7), nous obtiendrons l'équation:

$$
\frac{d \xi}{d t}=\frac{R}{B},
$$

qui conduit à la nouvelle intégrale des équations (7) qui dépend des fonctions elliptiques, précisément:

$$
t+l_{3}=B \int \frac{d \xi}{R}
$$

La troisième et la quatrième des équations ( 7 ) sont amenées au moyen des équations (9) à la forme:

$$
\left\{\begin{array}{l}
\frac{d \xi}{d t}=\frac{(a \xi-\xi) l_{1} \eta-\left(a \eta^{2}+\xi \xi\right) R}{B\left(1-\xi^{2}\right)} \\
\frac{d \eta}{d t}=\frac{\left(l_{1} \xi-\eta R\right)(\xi-a \xi)}{B\left(1-\xi^{2}\right)}
\end{array}\right.
$$


où

$$
a=\frac{B(C-A) x_{i} z_{0}}{A C Q_{0}^{2}}
$$

A l'aide de la deuxième des conditions (1) le carré de la quantité $a$ est exprimé au moyen des moments $A, B$ et $C$ de la façan suivante:

$$
a^{2}=\frac{(A-B)(B-C)}{A C}
$$

le signe de la quantité $a$ est opposé au signe du produit $x_{0} z_{0}$.

Après aroir multiplié la deuxième des équations (12) par $i=\sqrt{-1}$ et après avoir additionné ensuite ces équations, nous obtenons:

$$
\frac{d v}{d t}=\frac{\left(R-l_{1} i\right)(a v-2 \zeta) v}{2 B\left(1-\zeta^{2}\right)}-\frac{a\left(\eta_{1} i+R\right)}{2 B}
$$

où $v$ est déterminé par l'une des équations (6).

Le mourement da solide que j'examine est entièrementi déterminé par le mouvement de deux points: 1) par le mouvement du centre de gravité $G$ relativement à l'espace immobile et 2) par le mourement du point $v$ qui déterrnine à son tour la translation du point $F$ et du segment $O P$ dans leur mouvement relatif dans le solide.

Le mouvement du point $G$ se détermine par le changement de deux angles: 1) par l'angle $\theta$ entre la direction de la gravité $O V$ et la ligne $O G, 2)$ par l'angle $\psi$ entre la ligne donnée immobile et horizontale $O P$ et la ligne mobile horizontale $O N$, située dans le plan $\xi O \eta$ (Fig. 1). Ce mouvement du point $G$ qui s'exprime au moyen des fonctions elliptiques du temps $t$, déterminées par l'équation (10), se produit d'après les règles du pendule sphériques (voyez l'article de Mr. N. E. Joukavsky).

La détermination du mouvement du point $v$, représentant une quantité complexe, qui satisfait à l'équation (14), est réduite par une transformation, désignée plus loin, ì la recherche de l'équation différentielle liuéaire à coefficients doublement periodiques uniformes.

\section{$\$ 3$.}

Mouvement du centre de gravité $G$ d'après la règle du pendule sphérique.

L'équation (10), qui détermine le cosinus $\xi$ de l'angle $\theta=\nabla 0 G$, montre que cet angle change dans un ordre qui correspond au mouvement du point pesant $G$ d'après la règle du pendule sphérique avec la tension d'un poids égal à $\varrho_{v}{ }^{2}: B$. Par conséquent la tension réelle du poids

$$
g=\frac{1}{M}
$$

change par rapport à $M \varrho_{0}^{2}: B$. 
Afin que le mouvement que j'examine soit possible, il est nécessaire de satisfaire à quelques conditions. Ce que nous devons considérer avant tout.

La quantité $\xi$, comme cosinus de l'angle $\theta=\nabla O G$, doit satisfaire les conditions:

$$
-1<\xi<+1,
$$

et la quantité dérivée $\frac{d \xi}{d t}$ doit être une quantité réelle et par conséquent l'inégalité

$$
R^{2}>0
$$

doit aroir lieu.

Supposons

et remarquons que

$$
f(\xi)=R^{2}
$$

$$
f(-\infty)>0, \quad f(-1)<0 \text { et } f(+1)<0,
$$

nous nous convainquons que les conditions (15) et (16) ne peuvent pas être remplies autrement que si l'équation $f(\xi)=0$ a trois racines réelles et si l'une d'elles $\xi_{1}$ se place entre $-\infty$ et -1 , et les deux autres $\xi_{2}$ et $\xi_{3}$ se placent entre -1 et +1 . Les inégalités:

$$
\xi_{0}<-1 \text { et } \Delta>0
$$

doivent exister pour que l'exécution de ces conditions soit possible. Dans ces inégalités

$$
\left\{\begin{array}{l}
\zeta_{0}=-\frac{l_{2}}{2 \varrho_{0}}, \Delta=g_{2}^{3}-27 g_{3}^{2} \\
g_{2}=\left(\frac{12 \varrho_{0}{ }^{2}+l_{2}^{2}}{3 \varrho_{0}}\right) \sqrt[3]{\frac{B^{2}}{4 \varrho_{0}}} \\
g_{3}=l_{1}{ }^{2}-\frac{2 l_{2} B}{3}+\frac{l_{2}^{3} B}{54 \varrho_{0}^{2}}
\end{array}\right.
$$

Supposons que les conditions (17) sont remplies de sorte que les trois racines $\xi_{1}, \xi_{2}, \xi_{3}$ du polynome $f(\xi)=R^{2}$ soient réelles et se placent d'après la quantité dans l'ordre suivant:

$$
\xi_{1}<-1<\xi_{3}<\xi_{3}<+1 \text {. }
$$

En même temps les inégalités suivantes

$$
\xi_{1}<\xi_{0}<\xi_{2}
$$

auront lieu et la quantite $\xi$ varie entre les limites:

$$
\xi_{2} \leqq \xi \leqq \xi_{3} \text {. }
$$

Choisissons le commencement da temps $t$ de façon que pour $t=0$ la quantité $\zeta$ coïncide avec $\zeta_{2}$. Au moyen de cette condition nous trouvons d'après l'équation (10) que:

$$
t=B \int_{\xi=}^{\zeta} \frac{d \zeta}{R} .
$$


Comme pendant la croissance de $t$ en partant de zéro la quantité $\xi$ doit grandir en partant de $\xi_{2}$, le radical $R$ doit être pris avec le signe + , tant que $\xi$ n'atteint pas sa plus grande valeur $\xi_{3}$.

Transformons ensuite les variables $\zeta$ et $t$ dans le but d'exprimer la quantité $\zeta$ et les autres quantités, qui y sont liées, an moyen des formes normales des fonctions elliptiques. Nous supposons que:

$$
\xi=-y \sqrt[3]{\frac{2}{B \varrho_{0}}}-\frac{l_{2}}{6 \varrho_{0}}, \quad t=B \tau \sqrt[3]{\frac{2}{B \varrho_{0}}} .
$$

Après la transformation nous aurons:

$$
\begin{gathered}
\tau=\int_{y}^{e_{2}} \frac{d y}{\pi} . \\
R=\sqrt{4 y^{3}-g_{2} y-g_{3}}=\sqrt{4\left(y-e_{1}\right)} \overline{\left(y-e_{2}\right)\left(y-e_{3}\right)} .
\end{gathered}
$$

Ici les quantités $g_{2}$ et $g_{3}$ sont déterminées par les expressions (18). Les quantités $e_{0}, e_{1}, e_{2}$ et $e_{3}$ sont liées aux quantités déjà nommées $\xi_{0}, \xi_{1}, \xi_{2}$ et $\xi_{3}$ par les équations

$$
\zeta_{k}=-e_{k} \sqrt[3]{\frac{2}{B \varrho_{0}}}-\frac{l_{2}}{6 e_{0}}, \quad(k=0,1,2,3) .
$$

Il s'ensuit d'après cette farme et d'après les inégalités $(19),(20)$ et (21) que:

$$
e_{1}>e_{0}>e_{2} \geqq y \geqq e_{3}
$$

Supposons

$$
T=\int_{y}^{\infty} \frac{d y}{R},
$$

et suivons les indications, adoptées dans le lỉvre de Halphen «Traité des fonctions elliptiques". Nous obtiendrons:

$$
y=\wp T, \quad R=-\varphi^{\prime} T .
$$

La période réelle et la période imaginaire de ces fonctions seront:

$$
\omega=\int_{e_{2}}^{+\infty} \frac{d y}{R} \text { où } \quad \omega^{\prime}=\int_{-\infty}^{e_{2}} \frac{d y}{R}
$$

et en même temps:

$$
\left.\int_{e_{2}}^{\infty} \frac{d y}{R} \equiv \omega+\omega^{\prime *}\right) \text {. }
$$

*) Les quantités, liées par le gigne $\equiv$, ne peuvent différer les nnes des autres que par des nombres entiers des périodes $2 \omega$ et $2 \omega^{\prime}$. 
Il s'ensuit de cette formule et des inégalités (24) et (27), que

$$
T \equiv \tau+\omega+\omega^{\prime}
$$

et que

$$
y=\wp\left(\tau+\omega+\omega^{\prime}\right) \text { et } R=-\wp^{\prime}\left(\tau+\omega+\omega^{\prime}\right) \text {. }
$$

Malgré la présence de la quantité imaginaire $\omega^{\prime}$ dans ces expressions, les fonctions $y$ et $R$ restent des quantités réelles et s'expriment par les formules suivantes, qui ne contiennent pas de quantités imaginaires*):

$$
\left\{\begin{array}{l}
y=\wp T=e_{2}+\frac{\left(e_{2}-e_{1}\right)\left(e_{2}-e_{3}\right)}{\wp \nu-e_{2}}, \\
R=-\wp^{\prime} T=\frac{\left(e_{2}-e_{1}\right)\left(e_{2}-e_{3}\right) \rho^{\prime} \tau}{\left(\wp \tau-e_{2}\right)^{2}} .
\end{array}\right.
$$

En outre nous avons les formules suivantes*):

$$
\wp\left(\boldsymbol{\omega}+\boldsymbol{\omega}^{\prime}\right)=e_{2}, \wp \omega^{\prime}=e_{3} .
$$

Ces expressions ainsi que celles de (32) montrent, qu'avec le changement du temps $\tau$ de 0 jusqu'à $\omega$ la quantité $y$ diminue de $e_{2}$ jusqu'à $e_{3}$ (en même temps la quantité $\xi$ augmente de $\xi_{2}$ jusqu'à $\xi_{3}$ et $R>0$ ); avec la variation ultérieure du temps $\tau$ de $\omega$ jusqu'à $2 \omega$ la quantité $y$ va croissant de $e_{3}$ jusqu'à $e_{2}$ (en même temps la quantité $\zeta$ va diminuant de $\xi_{3}$ jusqu'à $\xi_{2}$ et $R<0$ ).

Ainsi la fonction $y$ passe daus les moments $2 m \omega$ et $(2 m+1) \omega$, où $m$ est un nombre entier, par le maximun $e_{2}$ et le minimum $e_{3}$; la quantité dérivée $\frac{d y}{d \tau}$ passe dans les mêmes moments par zéro $\left(\operatorname{car} \beta^{\prime}\left(\omega+\omega^{\prime}\right)=0\right.$ et $\rho^{\prime} \omega^{\prime}=0$ ) et change de signe.

- En passant à la définition de l'angle $\psi$ qui se trouve entre la ligne horizontale immobile $O P$ (Fig. 1) et la ligne horizontale mobile $O N$, située dans le plan $\xi O \eta$, nous déterminerons d'abord les composantes $\Omega_{1}, \Omega_{2}$ et $\Omega_{3}$ de la vitesse de rotation du solide autour de l'axe instantané sur les axes $O \xi, O \eta$ et $O \xi$. Ces composantes seront évidemment:

$$
\Omega_{1}=\frac{z_{3}}{\varrho_{0}} p-\frac{x_{0}}{\varrho_{0}} r, \quad \Omega_{2}=q, \quad \Omega_{3}=\frac{x_{0}}{\varrho_{0}} p+\frac{z_{0}}{\varrho_{0}} r .
$$

L'équation (4) est réduite, après l'élimination de $p$ et de $r$, à la forme suivante,

$$
\Omega_{3}-a \Omega_{1}=0 \text {. }
$$

Si nous sorsentendons par $\Phi$ l'angle $v O \xi$, qui représente la longitude du point $\boldsymbol{V}$ (ou, autrement, une signification de l'amplitude de la quantité $v=\xi+i \eta$ ) et qui complète l'angle $N O \xi$ à l'angle droit, nous obtiendrons:

*) Halphen, t. I, p. 37. 


$$
\begin{aligned}
& \Omega_{1}=\sin \theta \cos \phi \cdot \frac{d \psi}{d t}+\sin \Phi \cdot \frac{d \theta}{d t} \\
& \Omega_{2}=\sin \theta \sin \Phi \cdot \frac{d \psi}{d t}-\cos \Phi \cdot \frac{d \theta}{d t} \\
& \Omega_{3}=\cos \theta \frac{d \psi}{d t}-\frac{d \Phi}{d t} .
\end{aligned}
$$

Après avoir exctus les expressions $\Omega_{1}$ et $\Omega_{3}$ dans l'équation (36), nous trourons:

$$
(\cos \theta-a \sin \theta \cdot \cos \phi) \frac{d \psi}{d t}-a \cdot \sin \phi \frac{d \theta}{d t}-\frac{d \Phi}{d t}=0 .
$$

Nous savons que les cosinus des angles des axes mobiles $O \xi, O \eta$ et $O \xi$ avec les axes immobiles $O P, O Q, O V$ s'expriment (au moyen des formules cornues de Euler) par trois angles $\Phi, \psi$ et $\theta$, nous trouvons pour les casinus $\xi, \eta$ et $\xi$ les expressions suivantes:

$$
\left\{\begin{array}{l}
\xi=\cos \Phi \cdot \sin \theta, \\
\eta=\sin \Phi \cdot \sin \theta, \\
\xi=\cos \theta .
\end{array}\right.
$$

Ces expressions ne renferment pas du tout l'angle $\psi$. Après les avoir substituées dans la première des équations (12) nous trouvons:

$$
-\sin \theta \frac{d \Phi}{d t}=\frac{l_{1}(a \sin \theta \cdot \cos \phi-\cos \theta)}{B \sin \theta}+a \sin \theta \cdot \sin \phi \cdot \frac{d \theta}{d \bar{t}} .
$$

Cette équation équirant à l'équation (14).

En éliminant des équations (37) et (39) la dérivée $\frac{d \Phi}{d t}$, nous trouvons:

$$
\frac{d \psi}{d t}=\frac{l_{1}}{b \sin \theta}=\frac{l_{1}}{B\left(1-\xi^{2}\right)} .
$$

L'expression (40) montre que l'angle $\psi$ change aussi arec le mouvement du point $G$ d'après la loi du pendule sphérique. Comme cette loi a été suffisamment étudiée par d'autres auteurs nous ne retiendrons pas plus longtemps l'attention du lecteur sur le mouvement du point $G$.

$$
\$ 4 .
$$

Propriétés de rotation du point $v$, caractérisées par les changements périodiques de ६. Equation linéaire différentielle anx coefficients donblement périodiques uniformes, déterminant le monvement du point $v$.

Ses intégrales multiformes.

Dans la détermination de rotation d'un solide un rôle important est joué non seulement par le point $G$, mais aussi par le point $v$, qui représente la quantité $v=\xi+i \eta$, déterminée par l'expression (14). 
La position du point $v$ sur le plan $\xi O \eta$ détermine la position correspondante du point $V$, qui est projeté sur le plan $\xi O \eta$ dans le point $v$, retrauchant dans la direction de la gravité le segment $O V=1$. Quand le point $v$ se mouve:dans le plan $\xi O \eta$, le point correspondant $V$ se mouve relativement au solide éxaminé dans la sphère $O$, décrite du centre $O$ par un rayon égal à 1 .

Voilà pourquoi nous prendrons le point $V$ pour représenter la quantité complexe $v=\xi+i \eta$ sur cette sphère $O$.

Le modole de $v=\xi+i \eta$ s'exprime ainsi:

$$
|v|=\sqrt{\xi^{2}+\eta^{2}}=\sqrt{1-\xi^{2}} .
$$

Par conséquent le point $v$, représentant sur le plan $\xi O \eta$ la quantité $v=\xi+i \eta$, doit se trouver sur la circonférence $E$, décrite du centre $O$ par le rayon

$$
r=\sqrt{1-\xi^{2}}
$$

et le point correspondant $\nabla$, représentant la même quantité sur la sphère $O$, doit être situé sur la circonférence $F$, qui se trouve dans l'intersection de cette sphère avec un plan parallèle au plan $\xi O \eta$ et à la distance de $\xi$ du point $O$.

Comme $\zeta$ est une fonction périodique avee la période $2 \omega$ relativement au temps $\tau$ et en plus paire, le point $V$ doit prendre place sur la même circonférence $F$ non seulement au moment $\tau$, mais aussi à chacun des moments:

$$
\pm \tau \pm 2 m \omega
$$

où $m$ est un nombre entier. Le point $v$ correspondant doit aux mêmes moments prendre place sur la même circonférence $E$.

Examinons les limites dans lesquelles se déplace la circonférence $F_{y}$ pendant le changement continuel de $\tau$ depuis - $\omega$ jusquà $+\omega$. La fonction $\zeta$ étant paire, les déplacements de la circonférence $F$ dans les intervalles depuis - $\omega$ jusqu'à 0 et de 0 jusqu'à $+\omega$ se produisent d'après la même loi, mais dans un ordre inverse, de sorte qu'il suffit d'examiner le mouvement de la circonférence $F$ dans l'intervalle de 0 jusqu'à $+\omega$. Dans cet intervalle la circonférence $F$ passe sans interruption de la position la plus élevée $F_{2}$, qui se trouve à la distance de $\xi_{2}$ du point $O$, à la position la plus basse $F_{3}$, qui se trouve à la distance de $\xi_{3}$ du point $O *$ ). Ainsi avec le changement du temps $\tau$ la circonférence $F$ change périodiquement de place à l'intérieur de la zone sphérique, enfermée dans les cercles paralleles $F_{2}$ et $F_{3}$.

*) Dans le solide donné le haut et le bas sont déterminés comme les directions positive et negative de l'axe $O \zeta$ (Fig. 1). 
Puisque la eirconférence $E$ est la projeption de la circonférence $F$ sur le plan $\xi O \dot{\eta}$, les translations de la eirconférence $E$ sont faciles à reconnaître par les déplacements de la circonférence $F$, et vice-versa. Il faut faire cependant quelques remarques par rapport à la position et à la translation de la circonférence $E$.

Si $l_{1}{ }^{2}<B l_{2}$, nous aurons: $\xi_{2}<0<\xi_{3}$. Dans ce cas les cercles parallèles $F_{2}$ et $F_{3}$ sont disposés de côtés différents de l'équateur $E_{0}$ (section de la sphère $O$ par le plan $\xi O \eta$ ). Afin de distinguer dạns ce cas pour les deux positions différentes de la circonférence $F$, situées à une distance égale du centre $O$, les positions correspondantes coïncidentes de la circonférence $E$, nous distinguerons deux côtés da plan $\xi O_{\eta}:$ le, côté de l'endroit et de l'envers et nous dessinerons sur ce plan la circonférence $E$ du côté où est située la circonférence $F$ correspondante, De cette façon la circonférence $E$ seule, dessinée du côté correspondant du plan $\xi O \eta$, correspondra à la position donnée de la circonférence $F$, et vice-versa. Quand, avec le changement da temps $\tau$, la circonférence $F$ passe par l'équateur $E_{0}$ d'un hémisphère à l'autre, la circonférence $E$ passe par la même circonférence $E_{0}$ d'un côté du plan $\xi O \eta$ à l'autre.

Si $l_{1}^{2} \geq B l_{2}$, nous avons: $0<\xi_{2}<\xi_{3}$. Dans ce cas les sections $F_{2}$ et $F_{3}$ se trouvent à un seul et même côté de l'équation $E_{0}$, et par conséquent il n'y a aucune nécessité d'examiner la iranslation de la circonférence $E$ d'un côté du plan $\xi O \eta$ sur l'autre (sur l'icverse).

Ainsi la circonférence $E$ balanee toujours périodiquement dans une surface plane à forme d'anneau [de deux côtés (avec $l_{1}{ }^{2}<B l_{3}$ ) ou d'un côté (avec $\left.l_{1}{ }^{2} \geqq B l_{2}\right)$ ], enfermé entre les circonférences $E_{2}$ et $E_{3}$. On doit toujours prendre la position initiale du point $v$ (avec $\tau=0$ ) sur la airconférence $E_{2}$. Puis avec le cours du temps $\tau$ le point $v$ doit se mouvoir dans la surface à forme d'auneau désignée, se trouvant sur la circonférence $E$.

Remarquons encore qa'il dérive des corrélations $\xi_{1}+\xi_{2}+\xi_{3}=\xi_{0}$ et des inégalités (20) que $\xi_{2}+\xi_{3}>0$. Donc dans tous les cas $\xi_{2}{ }^{2}<\xi_{3}{ }^{2}$, c. à d. que la section $F_{2}$ est toujours plus rapprochée da centre $O$ que la section $F_{3}$.

Convenons de dopner le nom de pöles de la sphère $O$ à ses points d'intersection arec l'axe $0 Z$. Il n'est pas difficile de remarquer que la zone sphérique, enfermée entre les circonférences $F_{2}$ et $F_{3}$, n'atteint les pôles de la sphère $O$ que suivant la condition $l_{1}=0$, quand l'équation $R^{2}=0$ a les racines +1 et -1 . Dans les autres cas cette zone sphérique n'atteint jamais les pôles de la sphère 0 .

Passant à l'équation (14), remplaçons y $\zeta$ et $t$ par les variables déjà nommées $y$ et $\tau$, pour lesquelles les expressions (23), (32) et (33) ont lien, et ensuite transformons cette équation (14) en supposant que: 
où

$$
\begin{aligned}
v & =\frac{-1}{2 a} \sqrt[3]{\frac{2}{B \varrho_{0}}}\left(\frac{\rho^{\prime} T-e_{0}}{\beta T-e_{0}}\right) \frac{d \lg w}{d \tau} \\
& =\frac{-1}{2 a} \sqrt[3]{\frac{4}{B^{2} \varrho_{v}^{2}}}\left(\frac{R+l_{1} i}{\zeta-\xi_{0}}\right) \frac{d \lg w}{d \tau}
\end{aligned}
$$

et

$$
e_{0}^{\prime}=+l_{1} i
$$

$$
T=\tau+\omega+\omega^{\prime} \text {. }
$$

Nous obtiendrons après la transformation l'équation linéaire:

$$
\frac{d^{2} w}{d \tau^{2}}-\frac{1}{2}\left(\frac{\wp^{2} T-e_{0}}{\gamma T-e_{v}}\right) \frac{d w}{d \tau}+a^{2}\left(\wp T-e_{v}\right) w=0,
$$

dont les coefficients sont des fonctions uniformes doublement périodiques, renfermant la quantité imaginaire $e_{0}$.

Remarquons que les quantités $e_{0}$ et $e_{0}^{\prime}$ peuvent étre désignées ainsi:

$$
e_{0}=\wp_{0} \text { et } e_{0}^{\prime}=\wp^{\prime} T_{0} \text {, }
$$

où.

$$
\text { (43') } T_{0}=\omega+\omega^{\prime}+\tau_{0}=\omega+\omega^{\prime}+\sqrt{-1} \int_{e_{0}}^{e_{2}} \frac{d y}{\sqrt{1\left(e_{1}-y\right)\left(y-e_{2}\right)\left(y-e_{3}\right)}} \cdot
$$

En considérant les points singuliers des intégrales de l'équation (42), nous vojons que ces coefficients se transforment en quantités infinies dans deux cas: 1) quand $\tau \equiv-\tau_{0}$ et 2) quand $\tau \equiv-\omega-\omega^{\prime}$. Par suite d'un examen plus minutieux nous voyons que les points singuliers des intégrales de l'équation (42) ne coïncident qu'avee les points de la deuxième catégorie, c. à. d. avec les points $\tau \equiv-\omega-\omega^{\prime}$; dans le domaine de chaque point $\tau \equiv-\tau_{0}$ les intégrales restent des quantités finies, continues et uniformes.

Les points singuliers des intégrales de l'équation (42) représentent sur le plan du variable $\tau$ les quantités de la forme:

$$
2 m \omega+2 m^{\prime} \omega^{\prime}-\omega-\omega^{\prime}
$$

où $m$ et $m^{\prime}$ sont des nombres extiers. Ces points sont situés aux sommets des angles droits, formés par deux systèmes de lignes parallèles: le premier système est formé par les lignes parallèles à l'axe de l'ordonnée qui passent par les points:

$$
\omega+2 m \omega,(m=0, \pm 1, \pm 2, \ldots)
$$

le second système est formé par les lignes parallèles à l'axe des abscisses (à l'axe de quantités réelles) qui passent par les points:

$$
\omega^{\prime}+2 m^{\prime} \omega^{\prime},\left(m^{\prime}=0, \pm 1, \pm 2, \ldots\right) \text {. }
$$

Cette régularité géométrique dans la disposition des points singuliers des intégrales de l'équation (42) présente l'une des propriétés essentielles de l'équation (42) et conduit elle même à des conclusions importantes. 
Si nous supposons sur le plan du variable $\tau$ un cercle, décrit autour d'une rectangle, par les sommets duquel sont représentées les quantités:

$$
\omega+\omega^{\prime},-\omega+\omega^{\prime},-\omega-\omega^{\prime}, \omega-\omega^{\prime}
$$

(Fig. 2), dans lintérieur de ce cercle il n'y aura pas de points singuliers des intégrales de l'équation (42). Par conséquent les intégrales de l'équation (42) doivent se développer par degrés entiers et positifs de $\tau$ en séries qui convergent dans le domaine de ce cercle.

Les formes fondamentales de ces séries sont examinées plus bas. Maintenant nous remarquerons seulement que ces séries peuvent servir pour le calcul des résolutions de l'équation (42) quand le temps $\tau$ varie entre les limites de convergence

$$
-\sqrt{\omega^{2}+\left(\omega^{2} i\right)^{2}} \text { et }+\sqrt{\omega^{2}+\left(\omega^{\prime} i\right)^{2}} \text {. }
$$

Ces limites de convergence satisfout

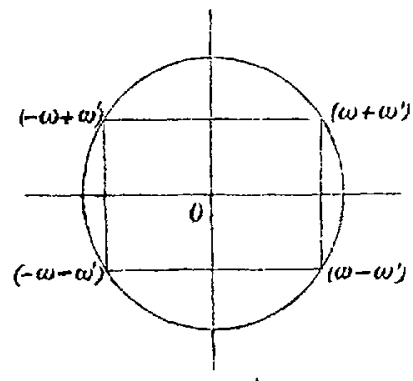

Fig. 2. au-de-là des traitons du problème de mécanique que nous examinons. Effectivement il suffit pour étudier le mouvement, que nous explorons, de savoir calculer les résolutions des équations (42) au moyen des séries désignées avec le changement de $\tau$ seulement dans les limites moins larges, c. à. d. $-\omega$ et $+\omega$; car on peut toujours réduire les calculs à ces limites à l'aide des substitutions linéaires correspondantes aux suppléments des périodes entières $2 \omega$.

Nous trouvons nécessaire de donner des à présent la forme générale des substitutions linéaires de ce genre, qui jouent un rôle essentiel dans le problème*).

Si $w_{1} \tau$ et $w_{2} \tau$ présentent deux résolutions diverses particulières de l'équation $(42), w_{1}(\tau+2 \omega)$ et $w_{2}(\tau+2 \omega)$ doivent posséder la même propriété et par conséquent il doit exister les corrélations:

$$
\left\{\begin{array}{l}
w_{1}(\tau+2 \omega)=x w_{1} \tau+\lambda w_{2} \tau, \\
w_{2}(\tau+2 \omega)=\mu w_{1} \tau+\nu w_{2} \tau,
\end{array}\right.
$$

où $x, \lambda, \mu$ et $\nu$ sont des coefficients constants. Pour faire le calcal de ces coefficients nous pouvons admettre dans les expressions (44) et
dans le résultat de leur différentiation que $\tau=-\omega$ et nous obtiendrons alors le système suivant des équations:

*) Dans l'article présent nous ne parlons pas des substitutions qui correspondent aux suppléments de la périoule imaginaire $2 \omega^{\prime}$; ces sabstitutions penvent avoir une signification grave dans la théorie genérale de l'equation (42), mais elles n'ont pas de rapport immédiat arec le problème mécanique. 
(45) $\left\{\begin{array}{l}w_{1} \omega=x w_{1}(-\omega)+\lambda w_{2}(-\omega), w_{2}^{\prime} \omega=\mu w_{1}(-\omega)+\nu w_{2}(-\omega), \\ w_{1}^{\prime} \omega=x w_{1}^{\prime}(-\omega)+\lambda w_{2}^{\prime}(-\omega), w_{2}^{\prime} \omega=\mu w_{1}^{\prime}(-\omega)+\nu w_{2}^{\prime}(-\omega) .\end{array}\right.$

Puisque les développements des fonctions $w_{1} \tau$ et $w_{2} \tau$ par les degrés de $\tau$ doivent converger avec $\tau= \pm \omega$, les quantités $\omega_{1}( \pm \omega)$, $w_{1}^{\prime}( \pm \omega), w_{2}( \pm \omega)$ et $w_{2}^{\prime}( \pm \omega)$, faisant partie des équations (45), peuvent être calculées à l'aide de ces développements; puis les coefficients $x, \lambda, \mu$ et $\nu$ peuvent être déterminés d'après les équations (45).

Plus bas nous aurons lieu d'éxaminer de différents côtés les propriétés des coefficients $\varkappa, \lambda, \mu$ et $\nu$ de la substitution, déterminée par les expressions (44) et qui donne la possibilité de calculer les fonctions $w_{1}(\tau+2 m \omega)$ et $w_{2}(\tau+2 m \omega)$ avec un nombre $m$ entier quelconque, si les fonctions $w_{1} \tau$ et $w_{2} \tau$ avec le changement de $\tau$ depuis $-\omega$ jusqu'à $+\omega$ sont connues.

Terminons ce paragraphe par l'explication d'une propriété analytique des intégrales générales des équations (2) d'après les conditions (1), laquelle propriété se trouve liée au développement des intégrales de l'équation (42) en séries infinies, convergente dans le domaine des points singuliers que nous avons nommés plus hant.

Ayant remarqué que

$$
\begin{gathered}
\wp T=\frac{1}{T^{2}}+\frac{g_{2}}{20} T^{2}+\frac{g_{3}}{28} T^{4}+\frac{g_{2}{ }^{2}}{2^{4} \cdot 3 \cdot 5^{2}} T^{6}+\frac{3 g_{2} g_{3}}{2^{4} \cdot 5 \cdot 7 \cdot 11} T^{8}+\cdots, \\
\frac{1}{2}\left(\frac{\wp^{0} T-\xi T_{0}}{\xi T-\xi T_{0}}\right)=\xi\left(T+T_{0}\right)-\xi T_{0}-\xi T, \\
\xi T=\frac{1}{T}+\int_{0}^{T}\left(\frac{1}{T^{2}}-\wp T\right) d T
\end{gathered}
$$

(Halphen, "Traité des fonctions elliptiques» t. I, p. 136-138), nous obtenons dans le domaine du point singulier $\tau=-\omega-\omega^{\prime}$ les développements suivants de deux intégrales indépendantes particulières de l'équation (42):

où

$$
\left\{\begin{array}{l}
w_{1}=T^{+a i}\left(1+c_{1} T+c_{2} T^{2}+c_{3} T^{3}+\cdots\right), \\
w_{2}=T^{-a i}\left(1+c_{1}^{\prime} T+c_{2}^{\prime} T^{2}+c_{3}^{\prime} T^{3}+\cdots\right),
\end{array}\right.
$$

$$
\begin{gathered}
T=\tau+\omega+\omega^{\prime}, \\
c_{1}=0, c_{2}=\frac{-a i \wp T_{0}}{4}, \quad c_{3}=\frac{-a i \xi p_{0}}{6(3+2 a i)}+\cdots, \\
c_{1}^{\prime}=0, c_{2}^{\prime}=\frac{ \pm a i \wp T_{0}}{4}, \quad c_{3}^{\prime}=\frac{+a i \rho^{\prime} T_{0}}{6(3-2 a i)}+\cdots
\end{gathered}
$$

On obtient les développements qui sont convergents dans le domaine du point singulier $\tau=2 m \omega+2 m^{\prime} \omega^{\prime}-\omega-\omega^{\prime}$, en remplaçant la quantité $T$ dans les formules (46) par la quantité

$$
T-2 m \omega-2 m^{\prime} \omega^{\prime} \text {. }
$$


Conme a est une quantité réelle, différente de zéro, nous voyous des expressions (46) que les intégrales de l'équation (42) doivent être des fonetions multiformes du variable $\tau_{\text {, }} \mathrm{D}^{\prime}$ ailleurs d'après l'expression (41), dans laquelle on doit supposer que :

$$
w=w_{1}+l_{4} w_{2} \text {, }
$$

où $l_{4}$ est une constante, nous voyons que la fonction $v$ et, par conséquent, les fonctions $p, q, r, \gamma, y^{\prime}$ et $\gamma^{\prime \prime}$, ayant l'arbitraire $l_{4}$, sont aussi des fonctions multiformes du variable $\tau$ ou dp tenps $t$.

Si partant de l'intégrale particulière (4) nous sommes parvenus à des fonctions multiformes, les intégrales gériérales des équations (2) suivant les conditions (1) conduiront à plus forte raison aux fonctions multiformes $p, q, r, \gamma, \gamma^{\prime}$ et $\gamma^{\prime \prime}$ du temps $t$. Ce résultat est conforme au résultat de Mme. Kovalevsky, qui affirme que les cas examinés par Euler, par Lagrange et par elle même sont les uniques, danø lesquels les quantités $p, q, r, \gamma, \gamma^{\prime}$ et $\gamma^{\prime \prime}$, déterminées au moyen des intégrales gémérales des équations (2), sont exprimées par des fonctions de temps uniformes, n'ayant, dans l'étendue finie du variable $\downarrow$, d'autres points singuliers que les póles.

La forme (5) des intégrales générales des équations (2) suivant les conditions (1) montre que les fonctions multiformes $p, q, \gamma, \gamma, \gamma^{\prime}$ et $\gamma^{\prime \prime}$ ont aussi, entre autre, en qualité de points singaliers, les pôles. Il reste à savoir ces pôles quand l'intégrale (4) a lieu. Il est facile de voir que ces pôles coïncident avec les points de la forme: $x \equiv-\tau_{0}$, où $\tau_{0}$ est déterminé par l'expression $\left(43^{\prime}\right)$. Effectivement ces points sont les pôles de l'expression

$$
\frac{p^{3} T-e_{0}^{2}}{89 T-\beta_{0}}
$$

qui est le multiplicateur de la seconde partie de l'expression (41). Cette expression détermine la quantité $v=\xi+i \eta$. Én plus, les pôles de la fonctian $v$ correspondent an zéro de la fonction $w$.

\section{$\S 5$}

Intégrales fondamentalos particulières de l'équation (42). Intégrales canoniques et propriétés caractéristiques do la quantité $h$. Trois cas de rotation do solide.

Nous nommerons intégrales fondamentales particulières de l'équation (42) les résolutions $w_{1} \tau$ et $w_{2} \tau$, qui satisfont les conditions:

$$
\left\{\begin{array}{l}
w_{1} 0=1, w_{1}^{\prime} 0=0, \\
w_{2} 0=0, w_{2}^{\prime} 0=i,
\end{array}\right.
$$

considérant $\tau=0$. Fixons notre attention sur la forme de ces résolations. 
la forme

$\mathrm{Si}$ nous exprimons l'intégrale générate des équations (42) par

$$
w=f \tau+i F \tau,
$$

où $f \tau$ et $F \tau$ sont des fonctions réelles, l'expression imaginaire conjuguée

$$
w=f \tau-i F \tau
$$

sera l'intégrale générate d'une autre équation

$$
\frac{d^{2} w}{d \tau^{2}}-\frac{1}{2}\left(\frac{\wp^{\prime} T+e_{0}^{\prime}}{\wp^{\prime} T-e_{0}}\right) \frac{d w}{d \tau}+a^{\prime}\left(\wp T-e_{0}\right) w=0,
$$

obtenue par l'équation (42) si nous remplaçons le coefficient imaginaire $e_{0}$ ' par une quantité conjuguée $-e_{0}^{\prime}$. Mais l'équation (49) se transforme en équation (42) après le remplacement de $\tau$ par $-\tau$; e'est pourquoi, après avoir subi le même changement, l'expression (48') se transforme de la façon suivante:

$$
w=f(-\tau)-i F(-\tau),
$$

qui présente sous une autre forme l'intégrale générale de l'équation (42). En combinant les expressions (48) et $\left(48^{\prime \prime}\right)$, nous pouvons aussi présenter l'intégrale générale de l'équation (42) sous la forme suivante:

$$
w=f \tau+f(-\tau)+i\{F \tau-F(-\tau)\} \text {. }
$$

Cette dernière forme de l'intégrale générale de l'équation (42) montre qu'il doit exister deux intégrales semblables particulières indépendantes de la même forme $(50)$, c. à. d. qu'elles ont dans leurs parties réelle et imaginaire les fonctions correspondantes paire et impaire. Si nous imposons à ces deux intégrales particulières les conditions (47), elles coïncideront avec les résolutions fondamentales. Par conséquent les intégrales fondamentales particulières de l'équation (42) se développent en séries suivantes:

$$
\left\{\begin{array}{c}
w_{1} \tau=1+\alpha_{2} \tau^{2}+\alpha_{4} \tau^{4}+\alpha_{6} \tau^{6}+\cdots \\
+i\left(\alpha_{3} \tau^{3}+\alpha_{5} \tau^{5}+\alpha_{7} \tau^{7}+\cdots\right), \\
w_{2} \tau=\beta_{2} \tau^{2}+\beta_{4} \tau^{4}+\beta_{6} \tau^{6}+\cdots \\
+i\left(\tau+\beta_{3} \tau^{3}+\beta_{5} \tau^{5}+\beta_{7} \tau^{7}+\cdots\right),
\end{array}\right.
$$

où $\alpha_{2}, \alpha_{3}, \ldots, \beta_{2}, \beta_{3}, \ldots$ sont des quantités réelles.

Le calcul successif des coefficients du développement (51) sera montré plus loin (dans le $\$ 10$ ).

Constituons les expressions des coefficients $x, \lambda, \mu$ et $\nu$ de la substitution (44), en supposant que $w_{1} \tau$ et $w_{2} \tau$ sont des résolutions fondamentales, c. à. d. les résolutions, déterminées par les expressions (51). Avec cette supposition nous aurons:

$$
\begin{aligned}
& w_{1}(-\omega)=\bar{w}_{1} \omega, \quad w_{1}^{\prime}(-\omega)=-\bar{w}_{1}^{\prime} \omega, \\
& w_{2}(-\omega)=\bar{w}_{2} \omega, \quad w_{2}^{\prime}(-\omega)=-\bar{w}_{2}^{\prime} \omega,
\end{aligned}
$$


où on sausentend en général par $\overline{2}$ une quantité conjuguée à $w$. II s'ensuit de ceci et des équations (45) que:

$$
\left\{\begin{array}{l}
x=\frac{1}{\delta}\left(w_{1} \omega \cdot \bar{w}_{2}^{\prime} \omega+\bar{w}_{2} \omega \cdot w_{1}^{\prime} \omega\right), \lambda=-\frac{1}{\delta}\left(\bar{w}_{1} \omega, w_{1}^{\prime} \omega+w_{1} \omega \cdot \bar{w}_{1}^{\prime} \omega\right) \\
\mu=\frac{1}{\delta}\left(w_{2} \omega \cdot \bar{w}_{2}^{\prime} \omega+\bar{w}_{2} \omega \cdot w_{2}^{\prime} \omega\right), \nu=-\frac{1}{\delta}\left(\vec{w}_{1} \omega \cdot w_{2}^{\prime} \omega+w_{2} \omega \cdot \bar{w}_{2}^{\prime} \omega\right),
\end{array}\right.
$$
où

$$
\delta=\bar{w}_{1} \omega \bar{u}_{2}^{\prime} \omega-\bar{w}_{2} \omega \bar{w}_{1}^{\prime} \omega .
$$

Il est facile de voir an moyen des expressions (52) et (52) que les quantités $*, \lambda, \mu$ et $\nu$ et celles, qui y sont conjuguées, satisfont aux. corrélations:

$$
\bar{x} \bar{\delta}=-\nu \delta, \quad \bar{\nu} \bar{\delta}=-x \delta, \bar{\mu} \bar{\delta}=\mu \delta, \quad \bar{\lambda} \bar{\delta}=\lambda \delta .
$$

La quantité $\delta$, qui se trouve dans les expressions précédentes et déterminée par l'expression (52), a une expression plus simple que l'on obtient de la manière suivante. Les résolutions particulières $w_{1} \tau$ et $w_{2} \tau$, déterminées par l'expression ( 51$)$, doivent d'après Liouville satisfaire à la condition:

$$
w_{1} \tau \cdot w_{2}{ }^{\prime} \tau-w_{2} \tau \cdot w_{1}^{\prime} \tau=i \sqrt{\frac{e_{0}-p_{0} T}{e_{0}-e_{2}}} e^{i \gamma \tau},
$$

où $\chi \tau$ esti une fonction réelle, déterminée par l'expression:

$$
x^{\tau}=\frac{e_{0}^{\prime}}{2 i} \int_{0}^{\tau} \frac{d \tau}{e_{0}-\gamma T}
$$

En supposant dans l'expression $\left(53^{\prime}\right) \tau=\omega$, nous obtiendrons, en passant aux quantités conjuguées, l'expression cherchée de la quantité $\delta$ :

$$
\delta=-i \sqrt{\frac{e_{0}-e_{3}}{e_{0}-e_{2}}} \cdot e^{-i \chi^{\mu}} .
$$

En même temps nous pouvons représenter les relations (53) ainsi:

$$
\begin{cases}\bar{x} \cdot e^{2 i \chi^{\omega}}=\nu, & \bar{\nu} \cdot e^{z i \chi^{\omega}}=x, \\ \bar{\mu} \cdot e^{2 i \chi \omega}=-\mu, & \bar{\lambda} \cdot e^{2 i \chi \omega}=-\lambda .\end{cases}
$$

Connaissant les quantités $x, \lambda, \mu$ et $\nu$, nous pouvons passer des résolutions fondamentales particulières de l'équation (42) à ses intégrales canoniques, qui sont souvent employées dans la théorie des équations linéaires aux coefficients uniformes périodiques ${ }^{*}$ ) et qui mènent notre probléme à des conclusions importantes.

*) Voir Floquet, «Sar les equations différentielles linéaires à coeffieients périodiques》 (Annales de l'Ecole normale supérieure, t. 13. 1883). 
En définissant les résolutions canoniques $W \tau$ de l'équation (42), pour lesquelles la condition

$$
W(\tau+2 \omega)=s W \tau
$$

a lieu, nous présenterons ce genre de résolution ainsi:

$$
W \tau=p^{\prime} w_{1} \tau+q^{\prime} w_{2} \tau \text {. }
$$

Ayant introduit cette expression $W \tau$ dans l'expression (54) et ayant recours an remplacement (44), nous obtenons une relation, d'où il $s^{\prime}$ ensuit que $p^{\prime}$ et $q^{\prime}$ satisfont aux équations

$$
\left\{\begin{array}{l}
(x-s) p^{\prime}+\mu q^{\prime}=0 \\
\lambda p^{\prime}+(\nu-s) q^{\prime}=0
\end{array}\right.
$$

Pour la compatibilité de ces équations la quantité $s$ doit satisfaire à l'équation carrée:

$$
s^{2}-(x+\nu) s+x \nu-\lambda \mu=0,
$$

qui a en général deux racines. Ainsi il existe en général deux résolutions de l'équation (42), satisfaisant la condition (54). Dans un cas particulier, quand les deux racines de l'équation (57) deviennent égales, ces deux résolutions de l'équation (42) deviennent coïncidentes. Si nous rejetons pour le moment ce dernier cas et si nons désignons par $s_{1}$ et $s_{2}$ les racines de l'équation (57) et par $W_{1} \tau$ et $W_{2} \tau$ les résolutions canoniques correspondantes de l'équation (42), nous avons les équations:

$$
\begin{aligned}
& \left\{\begin{array}{l}
W_{1}(\tau+2 \omega)=s_{1} W_{1} \tau, \\
W_{2}(\tau+2 \omega)=s_{2} W_{2} \tau,
\end{array}\right. \\
& W_{1} \tau=p_{1} w_{1} \tau+q_{1} w_{2} \tau \text {, } \\
& W_{2} \tau=p_{2} w_{2} \tau+q_{2} w_{2} \tau \text {, } \\
& q_{1}=\frac{v_{1}-x}{\mu} p_{1}=\frac{\lambda}{s_{1}-\nu} p_{1}, \quad q_{2}=\frac{s_{2}-x}{\mu} p_{2}=\frac{\lambda}{s_{2}-\nu} p_{2},
\end{aligned}
$$

dans lesquelles les quantités $p_{1}$ et $p_{2}$ peuvent être choisies arbitrairement. Nous supposerons que $p_{1}=p_{2}=1$; de sorte que nous aurons:

$$
\left\{\begin{array}{l}
W_{1} \tau=w_{1} \tau+q_{1} w_{2} \tau, \\
W_{2} \tau=w_{1} \tau+q_{2} w_{2} \tau,
\end{array}\right.
$$

où

$$
q_{1}=\frac{s_{1}-x}{\mu}=\frac{\lambda}{s_{1}-\nu}, \quad q_{2}=\frac{s_{2}-x}{\mu}=\frac{\lambda}{s_{2}-\nu} .
$$

Ayant remarqué que

$$
x \nu-\lambda \mu=-\frac{\vec{\delta}}{\delta}=e^{2 i \chi \omega},
$$

où $\chi \omega$ est déterminé par l'expression $\left(53^{\prime \prime}\right)$ considérant $\tau=\omega$, nous nous convainquons que les racines $s_{1}$ et $s_{2}$ de l'expression (57) penvent être exprimées ainsi: 


$$
s_{1}=\frac{x+\nu}{1+h^{2}}, \quad s_{2}=\frac{(x+\nu) h^{2}}{1+h^{2}},
$$

où

$$
\begin{gathered}
h= \pm N \pm \sqrt{N^{2}-1} \\
N=\frac{x+y}{2} \cdot e^{-i x \omega} .
\end{gathered}
$$

A l'aide des expressions ( $\left.53^{I V}\right)$ nous nous convainquons que la quantité $N$, déterminée par l'égalité (61'), et la quantité, qui y est conjuguée, sont égales, c. à. d.

$$
\bar{N}=N \text {. }
$$

Par là il s'ensuit que la quantité $N$ est réelle. En même temps la quantité $h$, déterminée par l'expression (61), doit être ou réelle (si $N^{2}>1$ ), ou (si $N^{2}<1$ ) imaginaire arec le mođule égal à 1 .

Par le choix youlu des signes dans la seconde partie de l'expreasion (61), qui détermine quatre siguifications de $h$, nous pourons obtenir qu'avec $N^{2}>1$ l'inégalité

$$
h>1
$$

ait lieu et qu'arec $N^{2}<1$ l'expression

$$
h=e^{\varphi i}
$$

ait lieu à condition que:

$$
0<\varphi \leqq \frac{\pi}{2} .
$$

La quantité $h$, choisie d'après la eondition (62) considérant $N^{2}>1$ et d'après les conditions (63) considérant $N^{2}<1$, sera nommée caractéristique.

La quantité caractéristique $h$, déterminée de cette façon, joue dans la suite un rôle essentiel soit comme constarite avec le mouvement donné, soit comme la fonction des quantités $l_{1}, l_{2}$ et $a$, pendant le changement continu d'un mourement à l'autre.

Disons dès maintenant, que la quantité caractéristique $h$, en qualité de fonction des variables $l_{1}, l_{2}$ et $a$, est continue dans les limites des changements, permis par les conditions du problème de mécanique que nous examinous, à part le cas où $\omega=\infty$, ce qui n'arrive que quand $l_{1}=0$ et $\xi_{0}=-1$. Effectivement les quantités $x, \lambda, \mu$ et $\nu$, déterminées par les expressions (52) et $\left(53^{\prime \prime \prime}\right)$, sont des fonctions finies et continues des quantités $l_{1}, l_{2}$ et $a$. De même que la quantité $N$, déterminée par l'expression $\left(61^{\prime}\right)$, doit être finie et continue; de là la continuité de la quantité caractéristique $h$ suivant les conditions désignées.

Si la quantité caractéristique $h$ est imaginaire, son amplitude $\varphi$, en qualité de fonction des quantités $l_{1}, l_{2}$ et $a$, conserve la continuité ainsi que $h$. 
D'après les conditions (63) elle ne surpasse pas la limite $\frac{\pi}{2}$, de sorte que cette limite est le mäximum de l'amplitude $\varphi$. C'est ce maximum qu'atteint successivement l'amplitude $\varphi$ croissante pendant les changements continus de $l_{1}, l_{2}$ et $a$, seulement $N$ devient zéro. Avec les changements ultérieurs de $l_{1}, l_{2}$ et $a$ qui occasionnent des changements la quantité $h$, l'amplitude $\varphi$ doit diminuer.

Pendant sa décroissance, l'amplitude $\varphi$ de la quantité imaginaire caractéristique $h$ peut s'approcher autant que l'on veut de zêro; quand $\varphi$ atteint zéro, la quantité $h$ devient 1 , les racines $s_{1}$ et $s_{2}$ deviernent égales. Avec les changements ultérieurs des quantités $l_{1}, l_{2}$ et $a$, qui exigent des changements dans $h$, cette quantité $h$ doit ou passer dans le domaine des significations réelles, croissant à partir d' 1 , ou retourner dans le domaine des significations imaginaires. Dans ee dernier cas l'amplitude $\varphi$, ayant atteint zéro en décroissant, doit croître sans interruption partant de zéro, comme de son minimum.

Nous royons de précédant, que le passage de la quantité caractéristique $h$ d'une signification réelle à une signification imaginaire (ainsi que d'une signification imaginaire à une réelle) est possible pendant les changements continues des quantités $l_{1}, l_{2}$ et $a$. L'un ou l'autre des passages est produit toujours par $h=1$ et est suivi du cas d'égalité des racines $s_{1}$ et $s_{2}$ que nous examinons plus bas.

Remarquons qu'au moyen des égalités (60) nous obtenons l'égalité

$$
h^{2}=\frac{s_{2}}{s_{1}}
$$

En exprimant l'intégrale générale de l'équation (42) à l'aide des résolutions canoniques $W_{1} \tau$ et $W_{2} \tau$, nous aurons:

$$
w=C_{1} W_{1} \tau+C_{2} W_{2} \tau,
$$

où $C_{1}$ et $C_{2}$ sont des constantes arbitraires. Ayant inclus cette expression $w$ dans l'équation (41), nous trouvons:

$$
v=P \tau \cdot \frac{W_{1}^{\prime} \tau+c W_{2}^{\prime} \tau}{W_{1} \tau+c W_{2} \tau}
$$

où

et

$$
c=C_{1}: C_{2}
$$

$$
P \tau=\frac{-1}{2 a} \sqrt[3]{\frac{2}{B \varrho_{0}}}\left(\frac{\beta^{\prime} T-e_{0}}{\wp^{2} T-e_{0}}\right)=\frac{-1}{2 a} \sqrt[3]{\frac{4}{B^{2} \varrho_{0}^{2}}}\left(\frac{R+l_{1} i}{\zeta-\zeta_{0}}\right) .
$$

La constante $c$ est choisie selon la dépendance de la position initiale du point $v$, qui, suivant $\tau=0$, doit absolument se trouver sur la circonférence $E_{2}$ (voyez le $\S 4$ ).

Supposons que le temps $\tau$ reçoive un accroissement égal au nombre entier $m$ des périodes $2 \omega$ et admettons que la fonetion $v$ acquière 
la signification $v_{m \text { dans }}$ le moment $\tau+2 m$. Comme en vertu des expressions (58)

$$
\left\{\begin{array}{l}
W_{1}(\tau+2 m \omega)=s_{1}{ }^{n} W_{1} \tau, \\
W_{2}(\tau+2 m \omega)=s_{2}{ }^{m} W_{2} \tau
\end{array}\right.
$$

nous aurons

$$
v_{m}=P \tau \cdot \frac{W_{1}^{\prime} \tau+c h^{2 m} W_{2}^{\prime} \tau}{W_{1} \tau+c h^{2 n} \frac{W_{2} \tau}{2}}
$$

où $h$ est la quantité caractéristique qui vient d'être désignée. L'équation (67) détermine le mouvement du point $v$ dans la phase du temps $\tau+2 m \omega$ et conduit à beaucoup de conséquences importantes.

Avant tout il faut remarquer que l'équation (67) constitue entre les quantités $Z=v_{* h}$ et $z=h^{2}$ une relation représentée parl'expression

$$
Z=P \tau \cdot \frac{W_{1}^{\prime} \tau+c z W_{2}^{\prime} \tau}{W_{1} \tau+c z W_{2} \tau}
$$

et connue sous le nom de transformation linéaire. Cette relation possède beancoup de propriétés remarquables qui reçoivent une expression géométrique simple, si l'on désigne les quantités $Z_{4}$ et $z$ par des points sur le plan des coordonnées angulaires. La propriété géométrique fondamentale de la transformation (68) consiste en ce qui suit: pendant le mouvement d'un des points $Z$ et $z$ sur la circonférence, l'autre point aussi doit se mouvoir sur une circonférence. Quelque fois l'une des deux circonférences peut se développer en ligne droite, qui peut être considérée comme une eirconférence dont le centre est éloigné à l'infini.

Choisissans parmi les propriétés de la transformation linéaire (68) celle que nous nommerons similitude de la disposition des points $Z$ et $Z$, qui représentent les quantités $Z$ et $z$, liées par la coordonnance (68). Si le point $z$ décrit la ligne courbe $l$, le point $Z$ en décrira wae autre $L$. Nous nommerons ces lignes $l$ et $L$ lignes correspondantes. Admettons que les deux lignes courbes $L$ et $l$ sont finies et continues. La similitude de la disposition des points correspondants $Z$ et $z$ s'exprime par la conservation d'une successivité égale dans la disposition de ces points sur l'étendue des courbes correspondantes $L$ et $l$. Ou, autrement dit, si l'on prend sur la courbe $l$ les points $z_{1}, z_{2}, z_{3}, \ldots$, qui se disposent sur son étendue d'après la succession des index, les points correspondants $Z_{1}, Z_{2}, Z_{3}, \ldots$ se disposeront le long de la courbe $L$ d'après la même succession des index. Cette propriété de la transformation (68) est le résultat d'une autre propriété, qui appartient à cette transformation ainsi qu'à beaucoup d'autres, et elle est cónnue sous le nom de similitude des parties infiniment petites.

Dans les paragraphes suivants ces propriétés nous serviront de base pour beaucoup de déductions. 
Ecrivons l'équation (67) ainsi:

$$
v_{m}\left(W_{1} \tau+c h^{2 m} W_{2} \tau\right)-P \tau\left(W_{1}^{\prime} \tau+c h^{2 m} W_{2}^{\prime} \tau\right)=0
$$

et donnons au nombre $m$ quatre significations: $m, m^{\prime}, m^{\prime \prime}$ et $m^{\prime \prime \prime}$. Nous aurons quatre relations, qui seront linéaires et homogènes relativement aux quatre quantités:

$$
W_{1} \tau, W_{2} \tau, W_{1}^{\prime} \tau, W_{2}^{\prime} \tau
$$

Par conséquent le déterminant de ces correlations linéaires doit être zéro, c. à. d.

$$
\left|\begin{array}{lllll}
v_{m}, & v_{m} & h^{2 m}, & 1, & h^{2 m} \\
v_{m^{\prime}}, & v_{m^{\prime}} & h^{2 n^{\prime}}, & 1, & h^{2 m^{\prime}} \\
v_{m^{\prime \prime}}, & v_{m^{\prime \prime}} & h^{2 m^{\prime \prime}}, & 1, & h^{2 m^{\prime \prime}} \\
v_{m^{\prime \prime \prime}}, & v_{m^{\prime \prime}} & h^{2 m^{\prime \prime \prime}}, & 1, & h^{2 m^{\prime \prime}}
\end{array}\right|=0 .
$$

Au moyen de quoi il est facile d'exprimer $v_{m}$ par les quantités $v_{m^{\prime}}, v_{m^{\prime \prime}}, v_{m^{\prime \prime}}$ et $h^{2}$. Il est aisé de voir que l'on peut exéeuter la construction du point $v_{m}$ avec une règle et un compas, si ce tablean des quatre quantités: $v_{m^{\prime}}, v_{m^{\prime \prime}}, v_{m^{\prime \prime}}$ et $h^{2}$ nous est donné.

La relation (68') prend une forme plus simple, si les nombres entiers $m, m^{\prime}, m^{\prime \prime}$ et $m^{\prime \prime \prime}$ sont successifs. Arrêtons notre attention sur ce cas et supposons que:

$$
m^{\prime \prime \prime}=m^{\prime \prime}+1=m^{\prime}+2=m-1 \text {. }
$$

Suivant ces conditions la corrélation $\left(68^{\prime}\right)$ est reduite à la forme

$$
\frac{\left(v_{m}-v_{m-2}\right)\left(v_{m-1}-v_{m-3}\right)}{\left(v_{m}-v_{m-1}\right)\left(v_{m-2}-v_{m-3}\right)}=\frac{\left(1+h^{2}\right)^{2}}{h^{2}}
$$

Prenant cette relation comme base, si nous connaissons $h^{3}$ et trois significations successives de $v_{m}$ (par exemple $v_{0}, v_{1}, v_{2}$ ), nous obtiendrons tous les points successifs

$$
\ldots, v_{-2}, v_{-1}, v_{0}, v_{1}, v_{2}, \ldots
$$

Si $h^{2}$ n'est pas donnée immédiatement, il suffit de savoir quatre positious du point $v_{m}$ pour l'obtenir. Aiusi s'il nous est donné la position des points $v_{0}, v_{1}, v_{2}$ et $v_{3}$, en supposant dans la corrélation (68') $m=3$, nous aurons l'expression

$$
\frac{\left(v_{3}-v_{1}\right)\left(v_{2}-v_{0}\right)}{\left(v_{3}-v_{2}\right)\left(v_{1}-v_{0}\right)}=\left(h+\frac{1}{h}\right)^{2}
$$

par laquelle il est facile d'obtenir $h^{2}$. Ainsi ou peut juger de la quantité caractéristique de $h$, en prenant pour base l'observation d'un mouvement pendant un intervalle égal à $6 \omega$.

Passons maintenant au cas des racines égales de l'équation (57) $\left(s_{1}=s_{2}=s\right)$, c. à. d. an cas pour lequel la condition

$$
(x+\nu)^{2}-4(x \nu-\lambda \mu)=0
$$


a lieu, ou, autrement dit, quand $N^{2}=1$. La quantite caractéristique $h$ se change en 1 . Les résolutions canoniques $W_{1} \tau$ et $W_{2} \tau$ caractérisées par ce qu'elles satisfont aux conditions (54) cessent d'être différentes, c. à.d. que dans le cas que j'examine il h'y a que la seule résolution $W_{1} \tau=W_{2} \tau=W \tau$; qui satisfasse la condition (54). La secopde résolntion, qui manque, est remplacée dans ce cas par la résolution de $W_{3} \tau$, qui satisfait la condition

$$
W_{3}(\tau+2 m \omega)=s^{m}\left[W_{3} \tau+2 m \omega W_{1} \tau\right]
$$

où $s$ est la racine de l'équation (57) et $m$ est un nombre entier. Afin d'obtenir la résolution exigée $W_{3} \tau$, on peut se servir de son expression faite à l'aide des résolutions fondamentales $w_{1} \tau$ et $w_{2} \tau$, justement:

$$
W_{3} \tau=p_{3} w_{1} \tau+q_{3} w_{2} \tau \text {. }
$$

Ayant introduit cette expression $W_{3} \tau$ et l'expression $W_{1} \tau$, déterminée par la première des équations $\left(58^{\prime}\right)$, dans l'expression (70), prise avec $m=1$, et nous servant ensuite des relations (44), nous obtiendrons une identité, d'où nous pourrons déduire que:

$$
\left\{\begin{array}{l}
(x-s) p_{3}+\mu q_{3}=2 \omega s \\
\lambda p_{3}+(v-s) q_{3}=2 \omega s q_{1}
\end{array}\right.
$$

$$
s=\frac{x+p}{\mu} \text { et } q_{1}=\frac{\nu-x}{\mu} .
$$

Les équations (72) ne différent l'ane de l'autre que par le multiplicateur commun; c'est pourquoi une des quantités $p_{3}$ et $q_{3}$ reste arbitraire. Supposons que $p_{3}=0$, de sorte que nous aurons:

$$
W_{3} \tau=q_{3} w_{2} \tau
$$

où

$$
q_{3}=\frac{2 \omega s}{\mu}=\frac{2 \cos q_{1}}{\nu-s}
$$

Après avoir choisi ainsi $W_{3} \tau$, nous obtiendrons pour la détermination de la quantité $v$ l'expression:

$$
v=P_{\tau} \cdot \frac{W_{1}^{\prime} \tau+c W_{3}^{\prime} \tau}{W_{1} \tau+c W_{3} \tau}
$$

où $c$ est une constante, déterminée par la position initiale du point $v$ sur la circonférence $E_{2}$ (dans le moment $\tau=0$ ). Si $\tau$ reçoit l'accroissement de $2 m \omega$, où $m$ est un nombre entier, la quantité $v$ se change en $v_{m}$, quantité, qui, suivant l'expression (70) et l'expression

$$
W_{1}(\tau+2 m \omega)=s^{m} W_{1} \tau,
$$

s'exprimera de la façon suivante:

$$
u_{m}=P \tau \cdot \frac{W_{1}^{\prime} \tau+c\left(W_{3}^{\prime} \tau+2 m \omega W_{1}^{\prime} \tau\right)}{W_{1} \tau+c\left(W_{3} \tau+2 m \omega W_{1} \tau\right)}
$$


Il résulte des remarques précédentes trois cas: 1) quand la quantité caractéristique $h$ est une quantité réelle, satisfaisante à la condition (62), 2) quand $h$ est une quantité imaginaire de la forme: $e^{g i}$, satisfaisante aux conditions (63), et 3) quand $h=1$. Dans les deux premiers cas le mourement du point $v$ est déterminé au moyen de l'expression (65) et dans le troisième cas, qu'on doit considérer comme cas transitoire par rapport aux deux premiers, le mouvement du point $v$ se dêtermine par l'expression (73).

Le tableau de rotation présente dans chacun de ces cas des particularités, qui se trouvent en relation arec la question concernant la possibilité on l'impossibilité de l'existence des mourements périodiques, produits par le point $v$, avec la période $2 \omega$; mouvements, déterminés par la formule (65), l'un d'après $c=0$ et l'autre d'après $c=\infty$. Nous avons pour le premier de ces mouvements périodiques:

$$
v=P \tau \cdot \frac{W_{1}^{\prime} \tau}{W_{1} \tau},
$$

pour le second mouvement nous avons:

$$
v=P_{\tau} \cdot \frac{W_{2}^{\prime} \tau}{W_{2} \tau}
$$

Examinons les trois cas que nous avons énoncés précédemment.

$$
\S 6 .
$$

Rotation du solide dans le cas, où le corps pesant est capable de produire deux mouvements asymptotiques périodiques avec la période $2 \omega$.

Arrêtons notre attention sur le cas où les racines $s_{1}$ et $s_{2}$ de l'équation (57) ne sont pas égales et où $h$ est une quantité réelle, qui satisfait la condition (62).

Supposons que la constante $c$ de l'expression (65), déterminée par la position initiale du point $v$, soit une quantité finie et différente de zéro. En examinant le mouvement du point $v$ dans la phase du temps, pendant lequel $\tau$ change en $\tau+2 m \omega$, nous devons avoir recours à la formule (67), qui détermine la position correspondante $v_{m}$ du point $v$. Cette formule nous démontre ce qui suit.

Si le nombre entier $m$ tend à $-\infty$, la quantité $h^{2 m}$ tend à zéro et le point $v_{m}$ tend à la position, pour laquelle nous avons

$$
v_{-\infty}=P \tau \frac{W_{1}^{\prime} \tau}{W_{1} \tau}
$$

Par conséquent le mouvement du point $v$, n'étant pas périodique pour le temps fini $\tau$, tend avec la décroissance infinie du temps $\tau$ an mouvement périodique avec la période $2 \omega$, lequel mouvement est déterminé au 
moyen de l'expression (75). Nommons ce mouvement périodique du point $\%$ et du point $V$ correspondant sur la sphère $O$ mourement asymptotique périodique de premier genre.

Si le nombre $m$ tend $\grave{a}+\infty$, la quantité $h^{9 m}$ tend $\grave{a}+\infty$ et le point $v_{m}$ tend à la position, pour laquelle nous arons

$$
v_{+\infty}=P \tau \frac{W_{2}^{\prime} \tau}{W_{2} \tau} \text {. }
$$

Par conséquent le mouvement du point $v$, n'étant pas périodique pour le temps fini $\tau$, tend avec la croissance infinie du temps $\tau$ au mouvement périodique avec la période $2 \omega$, lequel mouvement est déterminé au moyen de lexpression (76). Nommons ce mouvement périodique du point $v$ et du point correspondant $V$ sur la sphère $O$ mouvement asymptotique périodique de second genre.

Ainsi, quand le temps $\tau$ tend à $\pm \infty$, l'impériodicité du mouvement des points $v$ et $V$ se perd successivernent et ce mouvement devient périodique de tel ou tel genre dans les limites.

Il s'ensuit, en outré, que les rotations périodiques (75) et (76) sont possibles avec $h$ réelle et qu'on peut choisir les circonstances initiales de façon que chacune de ces rotations puisse se réaliser im. médiatement.

Il est facile de découvrir que le mouvernent périodique du point $v$, déterminé par l'expression (75), n'est pas stable par rapport au temps $\tau$ croissant infiniment, et que le mouvement pếriodique du point $v$, déterminé par l'expression (76), n'est pas stable par rapport au temps $\tau$ décroissant infiniment. Effectivement, par exemple, le mouvement périodique de premier genre, déterminé par l'expression (6⿹弋龴) avec $c=0$, devient, prenant $c$ aussi petite que l'ou veut, un mouvement agité impériodique, qui se développe en un mouvement périodique de deuxième genre avec le temps $\tau$ croissant sans limittes, et par conséquent s'éloigne beaucoup de la rotation périodique de premier genre.

La relation entre les quaptités:

$$
z=h^{2 m} \text { et } Z=v_{m},
$$

représentée par l'expression (67), appartient évidemment à la transformation linéaire (68). En même temps les quantités correspondantes $z$ et $Z$, déterminées par les expressious (79), sont disposées l'une sur l'axe $0 \xi$, l'autre sur la circonférence $E$ (voyez $\$ 4$ ). Pour cette raison et en vue de la propriété générale de la transformation (68), le point $Z$ doit se mouvoir sans interruption sur la circonférence $E$, pendant que le point $z$ change sa place sans interruption sur l'axe $O \xi$ des abscisses ou, pour mieux dire, la quantité $z$ change en gardant ses significations réelles.

Par là on remarque, en outre, que les mouvements mécaniques 
possibles du solide, que nous éxaminons, ne différant que par les positions initiales du point $v$ sur la circonférence $E_{2}$ (dans le moment $\tau=0$ ), se déterminent, ayant les autres circonstances initiales pareilles, par l'expression de la forme:

$$
v=P \tau \cdot \frac{W_{1}^{\prime} \tau+c z W_{2}^{\prime} \tau}{W_{1}^{\prime} \tau+c z W_{2} \tau}
$$

où $z$ est une quantité arbitraire constante réelle. Effectivement, si la quantité $z$ de la formule (80) n'était pas réelle, les positions du point correspondant $v$ ne se seraient pas placés sur la circonférence $E$ dans les phases du temps $\tau+2 m \omega$, ce qui aurait contredit les propriétés du module $v$, montrées au commencement du $\$ 4$.

Si dans l'expression (68) z change sans interruption de 0 à $+\infty$, le point $Z$ ne décrit sans interruption qu'une partie de la circonférence $E$, représentée par l'arc $v_{-\infty} v_{0} v_{+\infty}$. Comme on le voit d'après la similitude de la disposition des points $Z$ et $z$, déterminés par les expressions (79), les points $v_{m}$ doivent être disposés sur cet are et ils doivent y être disposés successivement dans l'ordre de la croissance de l'index $m$ (car les points correspondants, qui représentent les quantités $h^{2 m}$, se disposent sur l'axe $O \xi$ dans l'ordre de la croissance de l'exposant $m$ ). Les points correspondants $V_{m}$ de la sphère $O$ se disposent de la même façon sur l'are $\bar{V}_{-\infty} \nabla_{0} \nabla_{+\infty}$ de la circonférence $F$.

Le point $v$ ne se serait engagé sur l'arc, qui complète $v_{-\infty} v_{0} v_{+\infty}$ jusqu'à la circonférence entière $E$, qu'après avoir eu une autre position initiale, avec laquelle le signe de la constante arbitraire $c$ aurait changé (car le point $Z$ décrit cet arc complémentaire, quand $z$ change de 0 jusquà $-\infty$, c. à. $d$. quand $z$ a une signification négative).

Il n'est pas difficile de remarquer ce qui suit; quand le nombre $m$ tend à $-\infty$, le point $v_{m}$ tend au point $v_{-\infty}$ de sorte, que ses positions successives $v_{m}$ et $v_{m+1}$ se rapprochent de plus en plus, formant dans la limite sur l'arc $v_{-\infty} v_{0} v_{+\infty}$, près de $v_{-\infty}$, une ligne pointillée, composée d'une rangée de points infiniment rapprochés les uns des autres. Il $\mathrm{y}$ a le même rapprochement des points $v_{m}$ près des points $v_{+\infty}$ dans le cas où la quantité $m$ tend à $+\infty$. Les points $V_{m}$ forment des lignes pointillées auprès des points $V_{-\infty}$ et $V_{+\infty}$ sur l'are $V_{-\infty} V_{0} V_{+\infty}$ de la circonférence $F$.

Si nous supposons que le temps $\tau$ change sans interruption depuis - $\omega$ jusqu'à $+\omega$, les points $v_{m}$ décriront dans le plan $\xi O \eta$ des courbes $S_{m}$, et les points correspondants $V_{m}$ de la sphère $O$ décriront les courbes $\Sigma_{m}$. Nous n'arrêterons principalement notre attention que sur les courbes $\Sigma_{m}$, d'après lesquelles il est facile de se représenter la position et les propriétés des courbes $S_{m}$.

Chaque courbe $\Sigma_{m}$ commençe an point $U_{m}$ de le circonférence $F_{3}$, où 
se troure le point $V_{m}$ an moment $\tau=-\omega$; puis la courbe $\Sigma_{m}$, allant en haut, passe par l'intérieur de la zone sphérique entre les circonférences $F_{2}$ et $F_{3}$, en atteignant les circonférences $F_{2}$ dans le point, où se trouve le point mobile $V_{m}$ au moment $\tau=0$; puis la courbe $\Sigma_{m}$ retourne dans le domaine de la zone sphérique eptre les circonférences $\boldsymbol{F}_{2}$ et $\boldsymbol{F}_{3}$ et, baissant successivement, atteint au moment $\tau=+\omega$ la circonférence $F_{3}$ al point $U_{m+1}$, qui sert de commencement à la courbe $\Sigma_{m+1}$.

Les $\Sigma_{m}$ représentent, avep différentes valeurs de $m$, des morceaux d'une seule et même courbe sans interruption et ouverte $\Sigma$. Ces morceaux se relient sur la circonférence $F_{3}$ de sorte, que la fin du morceap $\Sigma_{m}$ soit le commencement du morceau $\Sigma_{m+1}$.

Sousentendans par $\boldsymbol{K}_{m}$ et $L_{m}$ les parties de la courbe $\Sigma_{m}$, décrites par le point $V_{m}$, l'une d'elles dans l'intervalle $\tau$ de $-\omega$ jusqu'à 0 et l'autre de 0 jusqu'à $+\omega$. Comme les points $V_{m}$ de la círconférence $F$ ne peuvent pas coïncider les uns avec les autres, les courbes

$$
\ldots, K_{-2}, K_{-1}, K_{0}, K_{1}, K_{2}, \ldots
$$

ne peuvent pas s'entrecroiser. De même que

$$
\ldots, L_{-2}, L_{-1}, L_{0}, L_{1}, L_{2}, \ldots
$$

ne peuvent pas s'entrecroiser. Mais les courbes $K$ peuvent se croiser arec quelques unes des courbes $L$.

Si les courbes $L_{m}$ et $K_{m}$ se croisent au point $\alpha$, les points $V_{m}$ et $V_{m^{\prime}}$ doivent prendre la position $a$ dans des moments égaux par leur quantité absolue et contraire par leur signe (car les points $\boldsymbol{V}_{m}$ et $\boldsymbol{V}_{m^{\prime}}$ peuvent entrer sur la même circonférence $F$ seulement dans les moments $\pm \tau$, pendant le changement de $\tau$ de $-\omega$ jusqu'à $+\omega$ ). C'est pourquoi le temps de l'introduction du point $V_{m}$ dans la position $\alpha$ est déterminé par léquation:

$$
v_{m} \tau=v_{m^{\prime}}(-\tau), \quad 0<\tau<+\omega,
$$

où, en général, $v_{m} \tau$ est la désignation abrégée de la fonction, qui se trouve dans la deuxième partie de l'expression (67).

Les courbes des limites $\mathcal{K}_{-\infty}$ et $\boldsymbol{K}_{+\infty}$ partagent la zone sphérique, renfermée entre les circonférences $F_{2}$ et $F_{3}$, en deux parties; dans l'une de ces parties se placent toutes les courbes $K_{m}$, se suivant dans l'ordre de l'accroissement de l'index $m$. L'autre partie de cette zone sphérique ne renferme pas de courbes $\boldsymbol{K}_{\mathrm{m}}$. Ces conclusions dérivent de la disposition des points $\nabla_{m}$ sur l'are $V_{-\infty} V_{0} V_{+\infty}$ de la circonférence $F$. Il résulte encore de l'examen de cette dísposition, que les courbes successives $K_{m}$ et $K_{m+1}$ se rapprochent de plus en plus les unes des autres, à mesure qu'elles s'approchent de chacone des positions de la 
limite $K_{-\infty}$ et $K_{+\infty}$, et forment près des courbes $K_{-\infty}$ et $K_{+\infty}$ une multitade de lignes serrées infiniment.

Cest de la même façon que se disposent successivement les courbes $L_{m}$ toutes dans une seule partie de la zone sphérique, séparée en deux par les courbes $L_{-\infty}$ et $L_{+\infty}$.

Les lignes $L_{m}$ et $K_{m}$ forment un filet par leur croisemeats réciproques dans les parties de la zone sphérique, qu'elles remplissent; ce filet a différentes épaisseurs dans différents endroits; il sera d'une épaisseur infinie près des points, où les courbes $K_{-\infty}$ et $L_{-\infty}$ et de même où les courbes $K_{+\infty}$ et $L_{+\infty}$ se relient.

La courbe $\Sigma$, qui est composée des morceaux $\Sigma_{m}$ ou, autrement, des courbes $K_{m}$ et $L_{m}$, est décrite entièrement par le point $V$ de la sphère $O$ avec le changement de $\tau$ depuis $-\infty$ jusqu'à $+\infty$. Quant anx morceaux de la limite $\Sigma_{\infty}$ et $\Sigma_{+\infty}$ de la courbe $\Sigma_{\text {, chacun d'eux }}$ représente une ligne fermée et est décrit d'une manière correspondante par le point $\gamma$ dans la période du temps $2 \omega$, si l'on peut toutefois admettre un des mourements correspondants asymptotiques périodiques du premier ou du deuxième genre, déterminés par les expressions (75) et (76).

Passons ì présent ̀̀ l'examen d'un nombre remarquable, qui caractérise les trajectoires $\Sigma, \Sigma_{-\infty}$ et $\Sigma_{+\infty}$; caractérise les accroissements de la longitude $\phi$ du point $V$, qui s'acquièrent pendant que le point $V$ décrit les ares donnés.

Représentons sur la sphère $O$ une courbe fermée quelconque $\sigma$, qui ne passe pas par les pôles, c. à. đ. les points du croisement de cette sphère avec l'axe $O \xi$. Supposons, que le point mobile $V$ fasse le tour de cette courbe fermée dans la direction donnée jusqu'à son retour au point de sortie. Pendant ce monvement la longitude $\phi d u$ point $V$ ou, antrement, l'amplitude $\Phi$ de la quantité $v$, représentée sur la sphère $O$ par le point $V$, changera sans interruption. L'accroissement de cette amplitude $\Phi$ sera exprimé, après que le point mobile $V$ ait produit le tour, par le nombre entier $j$ des circonférences $2 \pi$, c. à. d. que cet accroissement sera égal à $2 j \pi$. Ce nombre $j$ exprimera en même temps le nombre des tours produits par la courbe fermée $\sigma$ autour de l'axe $0 \xi$; le signe da nombre $j$ montrera la direction dans laquelle se produisent ces tours. Autrement dit, $j$ exprime le nombre des tours, faits autour de l'axe $O \xi$ par le segment $O V$, pendant que le point $V$ fait le tour désigné de la courbe fermée $\sigma$.

Montrons dès à présent la propriété fondamentale suivante de ce nombre $j$. Le nombre $j$ des tours, faits par la courbe fermée $\sigma$ antour de l'axe $O \xi$, ne change pas, si nous déformons cette courbe en faisant changer la place ses parties sur la surface de la sphère $O$, admettant, avec cela, le changement de la longueur de cette courbe ('élasticité), 
mais n'admettant pas la rapture de ses parties et son passage par les pôles de la sphère 0 . On peqt, pour plus de clarté, se présenter la courbe $\sigma$, pendant ses déplacements, sous la forme d'un fil flexible, élastique et fermé, qui permet un déplacement libre par toute la surface de la sphère $O$, excepté par ses pôles, où le fil rencoptre un obstacle à son déplacement. On peut se représenter cet obstacle sous la forme d'une tige dure cylindrique infinie coïncidant avec l'axe $O \xi$; cette tige dure ne laisse pas passer un fil flexible par les pôles. Avec ces conditions le déplacement arbitraire sur la sphère $O$ du fil fermé $\sigma$, que nous examinons, présente la déformation de la courbe $\sigma$, avec laquelle les nombres des tours, qu'elle produits antonr de l'axe $O Z$, ne peut changer.

Nous nommerons le déplacement de la courbe $\sigma$ suivant toutes ces conditions sa déformation continuelle. D'après la constance des nombres des tours, produits autour de l'axe $O \xi$ par le fil fermé, qui est déformé continuellement, ou peut déduire, que deux courbes fermées produisent le même nombre de tours autour de l'axe $0 \xi$, si elles peuvent être amenées à leur coïncidence par des déformations continuelles ou si elles peuvent être amenées à coïncider chacune séparément avec une troisième courbe fermée, soumise à une déformation continuelle.

Plus loin nous examinerons plusieurs courbes fermées, qui se composent des parties de la trajectaire $\Sigma$ et des arcs de la circonférence $F$ dans ses différentes positions. Voilà pourquoi il est indispepsable d'établir relativement aux arcs de la circonférence $F$ les indications uniformes suivantes.

Considérant les nombres $m^{\prime}$ et $m^{\prime \prime}$ comme des nombres entiers, supposons que $\tau$ reste invariable dans la deuxième partie de l'expression $(80)$, et que $z$ change continuellement de $h^{2 m^{\prime}}$ jusqu'à $h^{2 m}$. Sous ces conditions le point $v$, représentant la quantité déterminée par l'expression (80), décrira l'are de la circonférence $E$, enfermée dans les points $v_{m^{\prime}}$ et $v_{m^{\prime \prime}}$. Nous désignons justement l'arc correspondant de la circonférence $F$ sur la sphère $O$ par $V_{m^{\prime}} \nabla_{m^{\prime \prime}}$, et par $V_{\prime^{\prime}}{U_{m}^{\prime \prime}}$ nous désignerons l'are de la circonférence $F_{3}$ a arec laquelle coïncide l'arc $\nabla_{m^{\prime}} V_{m^{\prime \prime}}$ dans le mouvement $\tau=-\boldsymbol{\omega}$.

N'oublions pas ces indications et imaginons nous la courbe $\Sigma_{m}$, formant le morceau nommé de la trajectoire $\Sigma$, et l'arc $U_{m+1} U_{m}$ de la circonférence $F_{3}$. Ces deax courbes, prises ensemble, forment une courbe fermée que nous désignerons par $\sigma_{m}$. Donnant à $m$ des significations différenties entières, nous obtiendrons un groupe de courbes fermées de ce genre. Ces courbes joueront un rôle important dans notre théorie.

Si $l_{1}$ n'est pas un zéro, la zone sphérique, enfermée dans les circonférences $F_{2}$ et $F_{3}$, ne peut pas s'étendre jusqu'aux pôles de la sphère $O$ (comme il est démontré dans le $\$ 4$ ), et par conséquent les 
courbes fermées $\sigma_{m}$ ne peuvent pas passer par ces pôles. Au contraire ayant $l_{1}=0$, la zone sphérique désignée s'étend au moins jusqu'à un des pôles de la sphère $O$ et chaque courbe fermée $\sigma_{m t}$ passe tout an moins par un pôle. A cause de cela nous écarterons le cas $l_{1}=0$, en supposant que $l_{1}$ est différent de zéro, de sorte qu'aucune des courbes fermées $\sigma_{m}$ ne passera par les pôles de la sphère $O$.

Démontrons ensuite, que les courbes fermées

$$
\ldots, \sigma_{-2}, \sigma_{-1}, \sigma_{0}, \sigma_{1}, \sigma_{2}, \ldots
$$

font chacune le même nombre de tours autour de l'axe $O \xi$.

Disposons les courbes $\sigma_{m}$ de la déformation continuelle dans l'ordre suivant. Examinons une autre trajectoire, ayant pris, pour déterminer le mouvement du point $F$, l'expression $(80)$, qui correspond à une autre position initiale du point $v$ sur la circonférence $E_{2}$, et non pas l'expression (65). Le caractère de cette trajectoire reste le même et on peut supposer pour cette trajectoire des courbes fermées $\sigma_{m}(z)$, qui ont leur construction analogique aux courbes $\sigma_{m}$; mais à présent ces courbes $\sigma_{m}(z)$ dépendent de $z$; elles se déplaceront pendant le changement continuel de $z$, satisfaisant à toutes les éxigeances de la déformation continuelle, que nous avons nommée, et elles coincideront avec les courbes précédentes $\sigma_{m}$ ayant $z=1$. Supposons que $z$ croisse continuellement de 1 jusqu'à $h^{2}$. Il est évident qu'avec ce changement la courbe $\sigma_{m}(z)$, que nous déformons, conservera le nombre des tours autour de l'axe $O \xi$ et passera avec cela de sa position initiale $\sigma_{m}$ (ayant $z=1$ ) à la position finale (ayant $z=k^{2}$ ), qu'a occupée au commencement la courbe $\sigma_{m+1}$. On voit par là que les courbes $\sigma_{m}$ et $\sigma_{m+1}$ font le même nombre de tours autour de l'axe $O \xi$.

Après avoir montré ainsi la constance du nombre des tours, produits par chaque courbe fermée $\sigma_{m}$ autour de l'axe $O \xi$, désignons ce nombre constant par $J$ et nommons le le «index" da mourement, que nous examinous.

Quand $m= \pm \infty$ l'arc $U_{m} U_{m+1}$ tend à zéro. C'est pourqnoi la courbe $\sigma_{+\infty}$ change en courbe $\Sigma_{+\infty}$ et la courbe $\sigma_{-\infty}$ en $\Sigma_{-\infty}$. Il s'ensuit que les courbes fermées $\Sigma_{-\infty}$ et $\Sigma_{+\infty}$ produisent chacune un nombre de tours autour de l'axe $O \xi$ égal à l'index $J$. Autrement dit, si un des mourements périodiques, déterminés par les expressions (75) et $(76)$, a lieu, la ligne verticale $O V$ fait pendant ce mouvement et dans le période du temps $2 \omega$ le nombre $J$ de tours autour de l'axe $O \xi$.

Etendons la notion du nombre des tours, produits par la courbe autour de l'axe $O \xi$, jusqu'aux courbes ouvertes, tracées sur la sphère $O$. Déterminons, pour la courbe donnée de ce genre, le nombre $j$ des tours, qu'elle produit antour de l'axe $O \xi$, de la manière suivante: 


$$
j=\frac{\Phi_{i}-\Phi}{2 \pi},
$$

où $\left(\Phi_{1}-\Phi\right)$ est l'accroissement de l'amplitude $\Phi$ de la quantité $v$; cet

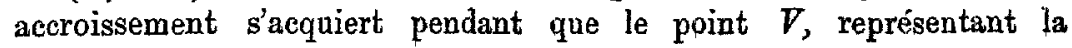
quantité $v$ de la sphère $O$, décrit la courbe donnếe.

Ensuite supposons que le mouvement impériodique du point $\nabla$, déterminé par l'expression (6̆), puisse être admis. Examinons le nombre $j_{k}$ des tours, produits autour de l'axe $0 \xi$ par la partie de la trajectoire $\Sigma$, qui est décrite par le point mobile $V$ dans un nombre entier $k$ de périodes $2 \omega$.

Démontrons que ce nombre $j_{k}$ peut toujours être représenté ainsi:

$$
j_{k}=J . k+\alpha,
$$

où $-1<\alpha<+1$. Représentons le moment, à partir duquel commence l'intervalle $2 \% \omega$, par la forme: $\tau=\tau^{\prime}+2 m \omega$, où $m$ est un nombre entier et $-\omega \leqq \tau^{\prime} \leqq+\omega$. Représentons ensuite les morceanx

$$
\widetilde{S}_{n,}, \widehat{S}_{m+1}, \ldots, \widetilde{S}_{m+k-1}
$$

de la trajectoire $\Sigma$, décrits par les points

$$
V_{m,}, V_{m+1}, \ldots, V_{m+k-1}
$$

pendant le changement du temps $\tau$ depuis $\tau^{\prime}$ jusqu'à $\tau^{\prime}+2 \omega$. Ce sont juste ces morceaux, qui sont décrits successivement par le point $V$ dans l'intervalle de $2 k \omega$, si l'on prend pour commencement le moment $\tau^{\prime}+2 m \omega$. Nous entendons par

$$
V_{m}^{\prime}, V_{m+1}^{\prime}, \ldots, V_{m+k}^{\prime}
$$

les positions des points

$$
V_{n}, V_{m+1}, \ldots, V_{m+k}
$$

dans le moment $\tau=\tau^{\prime}$; les morceaux désignés

$$
\varsigma_{m}, \varsigma_{m+1}, \ldots, \varsigma_{m+k-1}
$$

de la trajectoire $\Sigma$ se transforment en courbes fermées

$$
\hat{\xi}_{m}, \hat{亏}_{m+1}, \ldots, \hat{\jmath}_{m+k-1}
$$

par la jonction des ares carrespondants

$$
V_{m}^{\prime} V_{m+1}^{\prime}, \nabla_{m+1}^{\prime} V_{m+2,}^{\prime} \ldots, \nabla_{m+k-1}^{\prime} V_{m+k}
$$

qui sont décrits dans la direction opposée. Ces courbes fermées sont conduites, par la déformation continuelle de leurs parties, à la coüncidence avec les courbes correspondentes

$$
\sigma_{m}, \sigma_{m+1}, \ldots, \sigma_{m+k-1} \text {. }
$$

Telle est la déformation continuelle, que ces courbes subissent pendant le changement continuel de la quantité $\tau^{\prime}$ jusquà $-\omega$. A canse de cela chacune des courbes fermées

$$
\xi_{m}, \vec{s}_{m+1}, \ldots, \vec{\xi}_{m+k-1}
$$

fait le nombre $J$ de tours autour de l'axe $0 \xi$; prises ensemble elles 
produisent $J . k$ de tours. Pour obtenir à présent le nombre $j_{k}$, il ne reste, qu'à ajouter an nombre $J . k$ le nombre $\alpha$ des tours, produits autour de l'axe $O \xi$ par la joncion des arcs

$$
V_{m}^{\prime} V_{m+1}^{\prime}, \ldots, \nabla_{m+k-1}^{\prime} \mathcal{V}_{m+k}^{\prime}
$$

ou, autrement dit, par l'are $\nabla_{m}^{\prime} V_{m+k}^{\prime}$. Ainsi nous obtiendrons la formule (82), dans laquelle le nombre $\alpha$ des tour, produits autours de laxe $O \xi$ par l'arc $\nabla_{m}^{\prime} V_{m+k}^{\prime}$, doit être plus petit que 1 par sa quantité absolue (car l'are $V_{m}^{\prime} V_{m+k}^{\prime}$ ne peut faire qu'une partie d'un tour entier antour de l'are $O \xi$, puisqu'il ne compose qu'une partie de l'arc $V_{-\infty}^{\prime} V_{0}^{\prime} V_{+\infty}^{\prime}$ de la circonférence $F^{\prime}$ ). Le signe du nombre $k_{.} \alpha n^{\prime}$ est pas changé, quelque soit le $k$, pour la trajectoire donnée, et ne dépend que de la position initiale du point $v$.

Le nombre $\alpha$, que nous venons d'éxaminer, tend à zéro, quand, le nombre fini $k$ existant, le nombre $m$ tend à $\pm \infty$, car dans ce cas l'arc $V_{m}^{\prime} V_{m+k}^{\prime}$, proportionné au nombre $\alpha$, tend à zéro.

La formule (82) montre, que l'index $J$ a une signification essentielle dans la détermination de la partie entière du nombre des tours, produits dans lintervalle $2 k \omega$ par le point $V$. On peut dire la même chose des tours qui sont produits par la ligne verticale $O V$ autour de l'axe $O \xi$, dirigée vers le centre de gravité $G$. En plus la détermination de tout un nombre de circonférences entières $2 \pi$, qui se trouvent dans laceroissement relative de l'amplitude de $\Phi$ de la quantité $v$, dépend aussi de l'index $J$. En vue de cela il est important de pouvoir déterminer le nombre par lequel s'exprime l'index $J$ du mouvement que nous éxaminons.

Répondons à l'instant, que l'index $J$ du mouvement, que nous éxaminons, égale zéro. Cependant nous ne pouvons donner la preuve entière de cette thèse que plus loin (voyez le \$9). Ponr préparer les bases de la démonstration de cette supposition, remarquons ce qui suit.

On voit par l'examen de la déformation des courbes $\sigma_{m}(z)$, que nous arons étudiée, que l'index $J$ reste coustant, si nous passons d'un mouvement ì un autre en changeant la constante réelle $z$ de la formule (80) qui détermine le mouvement, c. à. d. si nous déplaçons la position initiale du point $v$ sur la circonférence $E_{2}$. D'ailleurs il n'est pas difficile de démontrer, que l'index $J$ reste constant encore, quand les quantités constantes $l_{1}, l_{2}$ et $a$ changent continuellement pendant le passage d'un mouvement à un autre, pourva que la quantité caractéristique $h$ reste réelle, on observeles conditions (17) et $l_{1}$ ne devient pas zéro. Effectivement des changements continuels de ce genre occasionnent des changements continus dans la quantité $h$ et dans les fonctions, qui déterminent la dosition du point $v$. Ces changements n'occasionnent que le déplacement de la courbe $\sigma_{m}$ sans la rupture de ses parties et sans que ces parties passent par l'axe $O \xi$. 
Ces déformations continuelles ne changent pas le nombre des tours de la courbe $\sigma_{u}$ antour de l'axe $O \xi$ et par conséquent elles ne changent pas l'index $J$ du mouvement.

Plus tard nous nous servirons de ces remarques pour base de la preuve du theorème, par lequel l'index $J$ da mourement est toujours zéro (voyez le \$9).

Supposons que $J=0$, et fixons notre attention sur quelques conséquences de cette supposition, lesquelles forment la particularité essentielle du cas, où la quantité caractéristique $h$ est une quantité réelle.

Si $J=0$, les courbes $\Sigma_{-\infty}$ et $\Sigma_{+\infty}$ ne font pas de tours autour de l'axe $O \xi$ et leurs projections $S_{-\infty}$ et $S_{+\infty}$ se disposent sur le plan $\xi O \eta$ d'une manière, présentée dans la figure 3 , où les courhes $S_{-\infty}$ et $S_{+\infty}$ sont représentées schérnatiquement par des lignes fermées $u u_{1} u_{2} u_{3}$ et $u^{\prime} u_{1}^{\prime} u_{2}^{\prime} u_{3}^{\prime}$. Pendant le mouvement $d u$ point $v$ le long de chacune de ces courbes, l'amplitude de la quantité correspondante $v$, déterminêe par l'angle $\xi O v$, ne peut jamais sortir hors les limites finies. Par conséquent, pendant la croissance illimitée du temps $\tau$, l'amplitade $\Phi$ de la quantité $v$, déterminée par chacune des expressions (75) et $(76)$, doit toujaurs waries. dans les limites fanies et dait garder une valeur fonie ayant $x=\infty$.

Cette conclusion s'étend jusqu'au mouvement impériodique du point $v$, déterminé par l'expression (65). Effectivement si $J=0$, le nombre $j_{k}$, proportional à l'accroissement de l'amplitude correspondante $\Phi$ de la quantité $v$ obtemu dans l'intervalle $2 \% \omega$, s'exprime, comme l'expression (82) le montre, ainsi:

$$
j_{k}=\alpha,-1<\alpha<+1
$$

Ce nombre $j_{k}$ reste fini arec n'importe quelle nombre de $h$; e'est pour. quoi l'amplitude $\Phi$ de la quantité $v$, déterminée par l'expression (65), doit toujours varier dans les limites finies pendant la croissance illimitée du temps $\tau$ et doit garder pane valeur finie quand $\tau=\infty$.

Nous nous servirons de ces remarques dans le $\$ 9$ après avoir démontrể le théorème, par lequel $J=0$. Ces remarques, entre antre, sont essentiellement importantes pour la déduction du postulat de Mr. B. Mlodzeiersky.

Apres avoir examiné les positions relatives des trajectoires des points $v$ et $F$, qui correspondent à différentes positions initiales du point $v$ sur la circonférence $E_{2}$, remarquons, qu'avec les conditions, que nous considérons, les racines $s_{1}$ et $s_{2}$ de l'équation $(57)$, exprimées 
par les expressions $(60)$, et les conjugnées $\bar{s}_{1}$ et $\bar{s}_{2}$ sont liées par les relations :

$$
\bar{s}_{1} \bar{\delta}=-s_{1} \delta, \quad \bar{s}_{2} \bar{\delta}=-s_{2} \delta .
$$

Au moyen de ces relations et de celles de (53) nous nous convainquons, que les quantités $q_{1}$ et $q_{2}$, déterminées par les expressions $\left(58^{\prime \prime}\right)$, et les quantités conjuguées remplissent les conditions:

$$
\bar{q}_{1}=q_{2}, \quad \bar{q}_{2}=q_{3},
$$

c. à. d. que les $q_{1}$ et $q_{2}$ sont des quantités imaginaires canjuguées. Les expressions $\left(58^{\prime}\right)$ et $\left(83^{\prime}\right)$ montrent que:

$$
W_{1}(-\tau)=\bar{W}_{2} \tau, \quad W_{2}(-\tau)=\bar{W}_{1} \tau .
$$

En dérivant ces relations, nous trouvons:

$$
W_{1}^{\prime}(-\tau)=-\bar{W}_{2}^{\prime} \tau, \quad W_{2}^{\prime}(-\tau)=-\bar{W}_{1}^{\prime} \tau \text {. }
$$

Les expressions (84) et (84') mènent à toate une suite de conclusions, concernant les trajectoires du point $v$ suivant des conditions différentes. Après avoir remarqué que suivant l'expression (66)

$$
\bar{P} \tau=-P(-\tau)
$$

nous trouverons d'abord les expressions:

$$
\left\{\begin{array}{l}
P(-\tau) \cdot \frac{W_{1}^{\prime}(-\tau)}{\bar{W}_{1}(-\tau)}=\bar{P} \tau \cdot \frac{\bar{W}_{2}^{\prime} \tau}{\bar{W}_{2} \tau} \\
P(-\tau) \cdot \frac{W_{2}^{\prime}(-\tau)}{W_{2}(-\tau)}=\bar{P} \tau \cdot \frac{\bar{W}_{1, \tau}}{\bar{W}_{1} \tau}
\end{array}\right.
$$

Ces expressions démontrent que les trajectoires $S_{-\infty}$ et $S_{+\infty}$, qui correspondent aux deux mouvements asymptotiques du point $v$, déterminés par les expressions (75) et (76), sont disposées symétriquement par rapport à $O \xi$; la position du point $v$ dans le moment $\tau$ sur la première trajectoire est symétrique avec la position du point $v$ dans le moment - $\tau$ sur la deuxième trajectoire. Les trajectoires correspondantes $\Sigma_{-\infty}$ et $\Sigma_{+\infty}$ du point $V$ de la sphère $O$ sont disposées symétriquement par rapport au plan $\xi O \xi$.

Examinons en second lieu les trajectoires du point $v$, pour lesquelles sa position initiale (dans le moment $\tau=0$ ) coïncide avec l'un des deux points du croisement de la circonférence $E_{2}$ avec l'axe $O \xi$. Nous avons pour ces positions: $v= \pm \sqrt{1-\xi_{2}^{2}}$. Par conséquent on choisit la constante $c$ pour les deux trajectoires, que nous examinons, à condition que

$$
\pm \sqrt{1-\xi_{2}^{2}}=P 0 \cdot \frac{W_{1}^{\prime} 0+c W_{2}^{\prime} 0}{W_{1} 0+c W_{2} 0}
$$

Cette équation est reduite à la forme: 


$$
\pm \gamma_{2}=\frac{q_{1}+c q_{2}}{1+c}
$$

où

$$
\gamma_{2}=\frac{\sqrt{1-\xi_{2}^{2}}}{i P_{0}}=\frac{2 a}{l_{1}} \cdot \sqrt[3]{\frac{B^{2} \rho_{0}^{2}}{4}} \cdot\left(\xi_{2}-\xi_{0}\right) \cdot \sqrt{1-\xi_{2}^{2}}
$$

La quantité $\gamma_{2}$ se détermine aussi par

$$
\gamma_{2}=a \varepsilon^{\prime} \sqrt{e_{0}-e_{2}}
$$

où $\varepsilon^{\prime}= \pm 1, l_{1} \varepsilon^{\prime}>0$. L'expression (86) conduit à deux valeurs de $\varphi$, savoir:

$$
c^{\prime}=-\frac{q_{1}-y_{2}}{q_{2}-\gamma_{2}}, \quad c^{\prime \prime}=-\frac{q_{1}+\gamma_{2}}{q_{2}+\gamma_{2}} .
$$

Au mojen des expression (83') nous nous convainquans que ces quantités $c^{\prime}$ et $c^{\prime \prime}$, ainsi que celles qui y sont conjuguées, satisfont less conditions $c^{\prime} c^{\prime}=1, c^{\prime \prime} \bar{c}^{\prime \prime}=1$. Par conséquent le module de chacune des quantités $c^{\prime}$ et $e^{\prime \prime}$ est 1 . Comme en plus les deux rotations, que nous étudions, font partie du cas général exprimé par l'ex pression (80), nous avons: $c^{\prime}=c z^{\prime}, c^{\prime \prime}=c z^{\prime \prime}$ et de là $c^{\prime}=c^{\prime \prime} z$, où $z$ eșt une quantité réelle, égale à $z^{\prime}: z^{\prime \prime}$. Dans le cas donné la quantité $z$ doit coïncider avec - 1; car dans le cas contraire les modules des quantités inégales $c^{\prime}$ et $c^{\prime \prime}$ ne pourraient pas cöncider chacun péparément avec 1. Ainsi $z=-1$, autrement $c^{\prime}=-e^{\prime \prime}$. A canse de quoi

d'où il s'ensuit que

$$
\frac{q_{1}+y_{2}}{q_{2}+y_{2}}=-\frac{q_{1}-y_{2}}{q_{2}-\gamma_{2}}
$$

$$
q_{1} q_{2}=\gamma_{2}^{2} \text {. }
$$

Comme d'après les expressions (83") les quantités $q_{1}$ et $q_{2}$ sont conjuguées, il en résulte des expressions $\left(87^{\prime}\right)$ que $\gamma_{2}{ }^{2}$ est le carré du module de chacune d'elles. C'est pourquoi on peut admettre:

$$
\left\{\begin{array}{l}
q_{1}=\gamma_{2} e^{\beta i}, \\
q_{2}=\gamma_{2} e^{-\beta i}
\end{array}\right.
$$

Nous aurons en même temps:

$$
c^{\prime}=-0^{\prime \prime}=e^{\beta i} \text {. }
$$

Par conséquent les deux monvements, que nous examinons, se déterminent ainsi :

$$
v=P \tau \frac{W_{1}^{\prime} \tau \pm e^{\beta i} W_{2}^{\prime} \tau}{W_{1} \tau \pm e^{\beta i}}
$$

Au moyen des expressions (84), (84') et (85) nous nous convainquons que la quantité $v$, déterminée par l'expression (89), change en une quantité conjuguée $\bar{v}$, en remplaçant $\tau$ par $-\tau$. Il s'ensuit que la trajectoire de chacun des mouvements du point $v$, auquel correspond, 
au moment $\tau=0$, la position du point $\nu$ sur l'intersection de laxe $O \xi$ aree la circonférence $E_{2}$, présente une courbe, pour laquelle l'axe $O \xi$ est un axe de symétrie. Le point $v$ occupe des positions symétriques sur cette trajectoire dans les moments $\tau$ et $-\tau$.

Dans l'expression (80) nous pouvons admettre $c=e^{\xi i}$, où $\beta$ est déterminé par (88). Nous aurons

$$
v=P \tau \frac{W_{j}^{\prime} \tau+e^{\beta i} z W_{2}^{\prime} \tau}{W_{1} \tau+e^{\beta i} z W_{2} \tau}
$$

où $z$ est une quantité arbitraire réelle. L'équation (89'), coïncidant avec l'équation $(65)$, concerne plus nettement une forme général de la constante arbitraire $c$ dans la formule (65), précisément: $c=e^{\beta i} z$. Ayant désigné la seconde partie de l'expression $\left(89^{\prime}\right)$ plus brièvement par $v(\tau, 2)$, au moyen des expressions (84), (84') et (85) nous nous convainquons que la fonction $v(-\tau, z)$ et la quantité conjuguée à la fonction

$$
v\left(x, \frac{1}{z}\right)
$$

coïncident. II s'ensuit que la trajectoire du point $v$, déterminée par l'expression $\left(89^{\prime}\right)$, ef la trajectoire du point $v$, déterminếe par l'expression

$$
v=P \tau \cdot \frac{W_{i}^{i} \tau+e^{\beta i} \frac{1}{z} W_{2}^{i} \tau}{W_{1} \tau+e^{\beta i} \frac{1}{z} W_{2} \tau},
$$

sont symétriques par rapport à $O \xi$. Le point $v$ occupe des positions symétriques sur l'une et l'autre des trajectoires dans les moments $\tau$ et $-\boldsymbol{x}$.

Nous examinerons deux mouvements particuliers du point $v$, déterminés par l'équation (89'). Pour ces deux mourements $z$ prend deux valeurs :

$$
+\frac{1}{h} \text { et }-\frac{1}{h} \text {. }
$$

Il' est facile de se convaincre par les expressions $\left(66^{\prime}\right),(84)$ et $\left(84^{\prime}\right)$, que la quantité $v$ prend pour chacun de ces mouvements des valeurs conjuguées au regard de $\tau=\omega+u$ et $\tau=\omega-u$. Conséquemment chacune des trajectoires correspondantes du point $v$ présente une courbe pour laquelle l'axe $O \xi$ est l'axe de symétrie, cependant le point $v$ occupe sur cette trajectoire des positions symétriques dans les moments $\tau=\omega+u$ et $\tau=\omega-u$. Chacune de ces deux trajectoires doit passer par le point correspondant du croisement de la circonférence $E_{3}$ avec l'axe $O \xi$; car le point $v$ doit se trouver sur la circonférence $E_{3}$ au moment $\tau=\omega$, de plus il doit être symétrique avec lui-même, c. à. d. qu'il doit se trouver sur l'axe $O \xi$ de la symétrie.

Ainsi nous n'avons que quatre trajectoires pareilles pour lesquelles l'axe $O \xi$ est laxe de symétrie. Chacune d'elles passe par le point correspondant du croisement des circonférences $E_{2}$ et $E_{3}$ avec l'axe $O \xi$. 


\section{$\S 7$.}

Rotation du solide dans le cas, où lo corps pesant n'est pas capable de reproduire des monvements asymptotiques periodiques avec la

$$
\text { période } 2 \omega \text {. }
$$

Supposons à présent que les racines $s_{1}$ et $s_{2}$ de P'expression (57) ne sont pas égales et que la quantité caractéristique $h$ est une quantité imaginaire de la forme: $h=e^{\varphi i}$. D'après cela l'amplitude $\varphi$ de la quantité $h$ satisfait les conditions (63).

En examinant le mouvement du point $v$, déterminé par l'expression (65), dans la phase du temps $\tau+2 m \omega$, nous devons avoir recours à l'expression (67), qu'on peut présenter ainsi:

$$
v_{m}=P \tau \frac{W_{1}^{\prime} \tau+c \cdot e^{2 m \varphi i} W_{2}^{\prime} \tau}{W_{1} \tau+e \cdot e^{2 \pi n \varphi i} W_{2} \tau} .
$$

La liaison entre les quantités

$$
Z=v_{n t} \text { et } z=e^{2 m \varphi i}
$$

représentée par l'expression (91), appartient aussi à la transformation linéaire (68). Les points $Z$ et $z$, déterminées par les expressions (92), sont disposés, avee différentes valeurs entières de $m$, le premier sur la circonférence $E$, et l'autre sur la circonférence $E_{0}$, décrite, partant du commencement des coordonnées, par le rayon égal à 1. Souvenonsnous de la propriété de la transformation linéaire (68) et concluons, qu'avec le déplacement continuel du point $z$ sur la circonférence $E_{0}$ le point correspondant $Z$, qui représente une quantité, déterminée par la formule (68), doit incessamment décrire la circonférence $E^{*}$ ).

Un tour entier du point $Z$ sur la circonférence $E$ doit correspondre à chaque tour entier du point $z$ sur la circonférence $E_{0}$. Si le point $z$ se mouve sur la circonférence $E_{0}$ dans une direction positive (dans la direction de l'accroissement de l'amplitude $z$ ), le point $Z$ se mourra sur la circonférence $E$ dans une direction déterminée, qui pent être quelquefois positive et quelquefois négative, c'est comment la direction de l'axe $O \xi$ est choisie. Admettons que la direction de l'axe

*) Dans le cas, où $\varphi=\frac{\pi}{2}$, les Équations (91) et (92) déterminent une seule paire de points $Z=v_{m}, m$ ayant tonte espèce de valeurs. Ce moyen n'éclaircit pas entièrement la position de la circonférence, par laquello se meut le point $Z$, quand $z$ se déplace le long de la circonférence $E_{0}$. Cependant la conclasion, que nous venons de faire par rapport anx changements continuels de $Z$ et qui est juste quand les significations de $\varphi$ approchent de $\frac{\pi}{2}$, doit garder aussi sa valeur dans la limite quand $\varphi=\frac{\pi}{2}$. 
$O \xi$ est choisie de façon que le mouvement da point $Z$ sur la circonférence $E$ se fait dans une direction positive*).

On voit au moyen des propriétés de la transformation linéaixe (68) qui relie les quantités (92), que toutes les rotations mécaniques possibles du solide, que nous étudions, ne différant que par les positions initiales $d u$ point $v$ sur la circonférence $E_{2}$, se déterminent, ajant toutes les autres conditions initiales égales, par l'expression

$$
v=P \tau \cdot \frac{W_{1}^{\prime} \tau+c \cdot e^{\vartheta i}}{W_{1} \tau+c \cdot e^{\vartheta i}} \frac{W_{2}^{\prime} \tau}{W_{2} \tau},
$$

où $\vartheta$ est une quantité constante arbitraire réelle. Effectivement si, dans la formule (93), la quantité \& n'était pas réelle, les positions du point $v$ dans les phases du temps $\tau+2 m \omega$, avec des $m$ différentes, ne se disposeraient pas sur la même circouférence $E$, ce qui contredirait les propriétés du module de $v$ que nous avons démontrées an commencement dans le $\$ 4$.

Comme l'expression $c e^{9 i}$ ne peut jamais devenir zéro ou infini pour les valeurs réelles de $\vartheta$, la formule (93) ne peut jamais coincider avec l'une ou l'autre des formules (75) et (76). C'est qu'arec les conditions présentes (c. à. d. avec $h$ imaginaire) pas un des mouvements periodiques du point $v$ avec la période $2 \omega$, déterminés par les $e x$ pressions (75) et (76), ne peut être produit dans le solide donné.

En sousentendant par $m^{\prime}$ et $m^{\prime \prime}$ des nombres entiers et par $v_{m^{\prime}}$ et $v_{m n^{\prime \prime}}$ des quantilés correspondantes, déterminées au moyen de l'expression (91), conrenons de sousentendre par $v_{m^{\prime}} v_{m^{\prime \prime}}$ l'are de la circonférence $E$ que le point $v$ décrit, pendant le changement continuel de $\vartheta$ de $2 m^{\prime} \varphi$ jusqu'à $2 m^{\prime \prime} \varphi$; le point mobile $v$ est déterminé par l'expression (9S), dans laquelle $\tau$ reste invariable. Nous désignerons l'arc correspondant de la circonférence $E$ sur la sphère $O$ par $V_{m^{\prime}} V_{m^{\prime \prime}}$; et nous designerons par $V_{m^{\prime}} U_{m^{\prime \prime}}$ l'arc de la circonférence $F_{3}$, avec laquelle l'arc $V_{m^{\prime}} V_{m^{\prime \prime}}$ coincide au moment $\tau=-\omega$.

Examinons le nombre $j$ des tours, produits par l'are $V_{m} V_{m+k}$ autour de l'axe $0 \xi$. Présentons la quantité $2 k \varphi$ ainsi

$$
2 k \varphi=2 k_{1} \pi+\varphi_{0},
$$

où $z_{1}$ est an nombre entier et $\varphi_{0}$ est choisi d'après les conditions:

$$
0 \leqq \varphi_{0}<2 \pi \text {. }
$$

* Ie changement de la direction de l'axe $O \xi$ est reduit au changement de la direction $O_{x}$; arec le ehangement de la direction positive de l'axe $O_{x}$ le signe de chacune des quantités $x_{0}, y, a$ et $p$ change et, comme le montrent les expressions (6), le signe de la quantité $\xi$ change anssi, c. à. d. que c'est la direction positive de l'axe $O \xi$ qui change. Quant amx signes des quantités $\eta$ et $\xi$, ils ne changent pas avec le changement indiqué de la direction de l'axe $O x$, comme on le voit au moyeu des expressions (6). 
L'expression (93') montre qu'avec le changement de $\vartheta$ de $2 m \varphi$ jusqu'à $2(m+k) \varphi$ le point $z=e^{\vartheta i}$ fait $k_{1}$ de tours complets sur la circonférence $E_{0}$ et plus il fait la partie du tour complet qui correspond au changement de $\vec{y}$ de $2\left(m \varphi+k_{1} \pi\right)$ jusqu'à $2\left(m \varphi+k_{1} \pi\right)+\varphi_{0}$. Par conséquent, d’après la propriété de la transformation linéaire (68) ou (93), le point correspondant $Z$ ou $v$ doit faire aussi $k_{1}$ de tour entiers et la partie $\alpha$ d'un tour entier sur la circonférence $E$. Le point correspondant $V$ de là sphère pression (93), et décrit l'are $V_{m} V_{m+k}$. On voit par là que le nombre cherché $j$ des tours, produits par l'arc $\nabla_{m} V_{m+\zeta}$ autour de l'axe $0 \xi$, doit être représenté ainsi;

$$
j=k_{1}+\alpha,
$$

où $0 \leqq \alpha<1$ et $k_{1}$ et un nombre entier, déterminé par l'expression $\left(93^{\prime}\right)$ suivant les conditions ( $\left.93^{\prime \prime}\right)$.

II est évident que quand $\varphi_{0}$ est petit, le nombre $\alpha$ doit être aussi petit. Si $\varphi_{0}=0, \alpha=0$.

Au moyen de $(63),\left(93^{\prime}\right)$ et $\left(93^{\prime \prime}\right)$ il est facile de se convaincre, que chacun des ares $V_{m} V_{m+1}$ et $V_{m} V_{m+2}$ ne fait qu'une partie d'un tour entier autour de l'axe $O \xi$.

Etablissons ensuite la notion de l'index de la rotation examinée, entièrement analogue à la nation correspondante, que nous avons expliquée dans le paragraphe précédent.

Représentons le morceau $\Sigma_{m}$ de la trajectoire $\Sigma_{\text {q }}$ décrit par le point $\nabla_{m}$ qui représente la quantité $v_{m}$, déterminée par l'expression
(91), pendant le changement de $\tau$ depuis $-\omega$ jusqu'à $+\omega$. Désignons ensuite par $\sigma_{m}$ une courbe fermée, composée de la courbe nommée précédemment $\Sigma_{n}$ et de l'arc $U_{m+1} U_{m}$ de la circonférence $F_{3}$, aree lequel l'arc $\nabla_{m+1} V_{n}$ coïncide dans le moment $\tau=-\omega$. Elargissons le cas $l_{1}=0$, car dans ce cas chaque courbe fermée $\sigma_{m}$ passe au moins par un des pôles de la sphère $O$.

Les courbes fermées

$$
\ldots, \sigma_{-2}, \sigma_{-1}, \sigma_{0}, \sigma_{1}, \sigma_{2}, \ldots
$$

font chacune un nombre égal de tours autonr de l'axe $0 \xi$. Pour démontrer cette sapposition, plaçons les courbes $\sigma_{m b}$ de la déformation continuelle dans l'ordre suivant. Examinons lautre trajectoire du point $V$, après avoir pris, pour déterminer sa rotation, non pas l'équation (65), mais la (93) qui correspond à une autre position initiale du point $v$ sur la circonférence $E_{2}$. On peut imaginer pour cette trajectoire des courbes fermées correspondantes $\sigma_{m}(9)$, qui ont leur construction analoge aux courbes $\sigma_{m}$. Les courbes $\sigma_{m}(\vartheta)$ coïncident arec les courbes $\left(94^{\prime}\right)$, quand $\vartheta=0$, et se déforment continuellement pendant la croissance de $\vartheta$ de 0 jusqu'à $2 \varphi$. Il est érident, qu'avec 
une déformation continuelle pareille la courbe $\sigma_{m}(\vartheta)$ passera de sa position initiale $\sigma_{m}$ (suivant $\vartheta=0$ ) à une position finale (quand $\vartheta=2 \varphi$ ), qui était occupée d'abord par la courbe $\sigma_{m+1} ;$ pendant son déplacement la courbe $\sigma_{m}(\vartheta)$ gardera invariable le nombre des tours qu'elle produit autour de l'axe $O \xi$. Il s'ensuit que les courbes (94') font chacune le même nombre de tours autour de l'axe $O \xi$.

Désignons ce nombre de tours par $J$ et nommerons le «index» du mouvement examiné. Il s'agit d'éclaircir à présent la question de la détermination de ce nombre, qui joue un grand rôle dans le mouvement que nous examinons.

Disons d'abord sans preuve que l'index $J$ est toujours égal à zéro dans le cas que nous examinons. Remettons la preuve entière de cette supposition jusqu'au $\S 9$. Maintenant arrêtons notre attention sur la constance de l'index $J$ avec le changement de quelques quantités constantes.

L'examen de la déformation ci-devant désignée des courbes $\sigma_{m}(\vartheta)$ montre, que l'index $J$ ne change pas, s'il passe d'un mouvement à l'autre, en changeant la constante $\vartheta$ dans l'équation (93), qui détermine le mouvement, c. à. d. en déplaçant la position initiale du point $v$ sur la circonférence $E_{2}$.

D'ailleurs il est facile de montrer, que la constance de l'index $J$ est gardée aussi pendant le changement de mourements, pendant lequel les constantes $l_{1}, l_{2}$ et $a$ changent constamment, si seulement la quantité caractéristique $h$ reste imaginaire, on observe les conditions (17) et la quantité $l_{1}$ ne devient pas zéro. Effectivement les changements continuels de ce genre produisent des changements continus dans la quantité $h$ et dans les fonctions qui déterminent la position du point $v$, c. à. d. que ces changements ne font qu'occasionner des déformations continuelles dans les courbes $\sigma_{m}$ (sans faire rompre leurs parties et sans les faire passer par l'axe $O \zeta$ ); ces déformations ne changent pas le nombre des tours, que produit la courbe $\sigma_{m}$ autour de l'axe $0 \xi$, et conséquemment ne changent pas l'index $J$.

Si par conséquent l'index $\mathcal{J}$ est un zéro pour la rotation donnée, pour laquelle les conditions (17) doivent être remplies, si la quantité $l_{1}$ est différente de zéro et si la quantité $h$ a une valeur imaginaire, l'index $J$ restera aussi zéro aux rotations, pendant lesquelles les constantes $l_{1}, l_{2}$ et $a$ acquièrent des significations contiguëes qui satisfont aux mêmes conditions. Nous nous servirons de cette remarque comme de base pour la preuve, qui montre, que l'index $J$ que nous examinons est toujours égàl à zéro (voyez le \$ 9).

Examinons maintenant le nombre $j_{k}$ des tours produits autour de l'axe $O \xi$ par la partie de la trajectoire $\Sigma$, décrite par le point $V$ 
dans l'intervalle $2 k \omega$, égal au nombre entier $k$ des périodes $2 a$. Montrons que ce nombre $j_{k}$ peut toujours être représenté ainsi:

$$
j_{k}=J \cdot k+k_{1}+\alpha,
$$

où $0 \leqq \alpha<1$ et $k_{1}$ est nombre entier, déterminé par l'expression (93) suivant les conditions $\left(93^{\prime \prime}\right)$. Fixons le moment, à partir duquel commence l'intervalle $2 k \omega$, de cette façon: $\tau=\tau^{\prime}+2 m \omega$, où $m$ est un nombre entier et $-\omega \leqq \boldsymbol{\tau}^{\prime} \leqq+\omega$. Représentons les morceaux

$$
\Im_{m}, \Im_{m+1}, \ldots, \Im_{m+k-1}
$$

de la trajectoire $\Sigma$, décrits par les points

$$
\nabla_{m}, \nabla_{m+1}, \ldots, V_{m+k-1}
$$

avec le changement de $\tau$ de $\tau^{\prime}$ jusqu'à $\tau^{\prime}+2 \omega$. Ce sont précisément ces morceaux quj sont décrits successivement par le point $\nabla$ dans l'intervalle $2 \pi \omega$ que nous arons examiné. Nous eptendons par

$$
V_{m,}, \nabla_{x+1}^{\prime}, \ldots, V_{m+k}^{\prime}
$$

les positions des points

$$
V_{m}, \nabla_{m+1}, \ldots, \nabla_{m+k}
$$

dans le moment $z=x^{\prime}$; les morceaux désignés

$$
\mathfrak{S}_{m}, \mathfrak{S}_{m+1}, \ldots, \mathfrak{S}_{m+k-1}
$$

de la trajectoire $\Sigma$ se transforment en courbes fermées

$$
\xi_{m}, \mathfrak{s}_{m+1}, \ldots, \mathfrak{\xi}_{m+k-1}
$$

par la jonction des ares correspondantes

$$
V_{m}^{\prime} V_{m+1}^{\prime}, \nabla_{m+1}^{\prime} V_{m+2}^{\prime}, \ldots, V_{m+k-1}^{\prime} \nabla_{m+k}^{\prime}
$$

de la circonférence $F^{\prime}$, ares qui passent dans la direction opposee. La déformation, que les courbes

$$
\hat{\Xi}_{m}, \tilde{\Xi}_{m+1}, \ldots, \hat{\Xi}_{m+k-1}
$$

subissent pendant le changement continuel de $\tau^{\prime}$ jusqu'à - $\omega$, n'occasionne pas la rupture de leurs parties et leur passage par l'are $O \xi$ et conduit ces courbes en cas limite, d'après $\tau^{\prime}=-\omega$, en coïncidence avec les courbes

$$
\sigma_{m}, \sigma_{m+1}, \ldots, \sigma_{m+k-1} \text {. }
$$

A cause de cela chacune des courbes fermées

$$
\xi_{m}, \Xi_{m_{i}+1}, \ldots, \hat{\zeta}_{m i+k-1}
$$

fait $J$ de tours autour de l'axe $O \xi$, et réunies ensemble elles font $J . k$ de tours. Pour obtenir le nombre $j_{k}$ il ne reste qu'à joindre au nombre $J . k$ le nombre $j$ des tours, produits autour de l'axe $O \xi$ par la réunion des arcs

$$
\nabla_{m}^{\prime}, \nabla_{m+1}^{\prime}, \ldots, \nabla_{m+k-1}^{\prime}, \nabla_{m+k}^{\prime}
$$

ou, autrement dit, par un are $\nabla_{m}^{\prime} \gamma_{m+k}^{\prime}$. Ce dernier nombre $j$ est déterminé par l'expression (94). De sorte que l'expression (95) se troure entièrement demontrée. 
Représentons l'expression (95) ainsi:

$$
j_{k}=\left(J+\frac{k_{1}}{k}\right) k+\alpha
$$

et examinons la dans la limite, quand $k$ tend à $\infty$. Par l'expression (93') suivant les conditions (93') on voit, que le rapport $\frac{k_{1}}{k}$ tend au nombre positive $\frac{\varphi}{\pi}$, qui d'après les inégalités (63) diffère de zéro et n'est pas plus grand que $\frac{1}{2}$. En même temps la somme

$$
J+\frac{k_{1}}{k}
$$

tend dans la limite à la quantité

$$
J+\frac{\varphi}{\pi}
$$

qui par sa quantité absolue ne peut pas etre plus petite que $\frac{\Phi}{\pi}$,

Arec ces conditions la seconde partie de l'expression (95') doit tendre, par sa valeur, à l'infini. Par conséquent le nombre absolu des tours, produits autour de l'axe $O \xi$ par la ligne $O V$, grandit indéfiniment dans l'intervalle croissant sans limites. Cette conclusion a lieu aussi quand $\boldsymbol{J}=0$.

Cette déduction montre que l'amplitude $\Phi$ de la quantitév, déterminée par l'expression (65), doit atteindre, pendant la croissance illimitée du temps $\tau$, la limite $\Phi=\infty$. Cette propriété des changements continuels de l'amplitude $\Phi$ forme la particularité essentielle du cas de rotation d'un solide que nous examinons, c. à. d. du cas où la quantité caractéristique $h$ est une quantité imaginaire. L'existence de cette propriété, $J$ étant zéro, est nécessaire et suffit pour que la quantité caractéristique $h$ soit imaginaire.

Les circonstances éxaminées existant, le solide n'admet pas, comme il a été demontré, les mouvements asymptotiques périodiques (75) et (76); cependant dans le cas que nous examinons le point $v$ peut aroir quelquefois des mouvements périodiques d'un autre caractère. Le mouvement des points $v$ et $V$ sera périodique, si lamplitude $\varphi$ de la quantité caractéristique $h$ est commensurable avec le nombre $\pi$. Effectivement nous pouvons dans ce cas représenter l'amplitude $\varphi$ ainsi:

$$
\varphi=\frac{x^{\prime}}{n} \pi
$$

où $n^{\prime}$ et $n$ sont des nombres entiers positifs premiers entre eux, satisfaisants l'inégalité: $n^{\prime}<2 n$ d'après les conditions (63). Quand $\varphi$ a cette valeur, nous voyons d'après les expressions (65) et (91) que $v_{n}=v$, c. à.d. que la fonction $v$, déterminée par l'expression (65), est 
périodique avec la période $2 n \omega$. En même temps le mouvement des points correspondants $v$ et $V$ doit être périodique avec la même période. Cette périodicité de mouvement a lieu avec toute position initiale du point $v$ sur la circonférence $E_{2}$. La trajectoire $\Sigma$ présente dans ce mouvement une courbe fermée, décrite entièrement par le point $V$ dans l'intervalle $2 n \omega$. Le nombre des tours, faits autour de l'axe $O \xi$ par la trajectoire $\Sigma$, ou, antrement, le nombre des tours, produits dans lintervalle $2 n \omega$ par la ligne $O F$ autour de la ligne $O \xi$, est déterminé au moyen des expressions (95) et (93'), dans lesquelles on doit admettre: $\vec{k}=n$ et où on doit attribuer à la quantité $\varphi$ la signification (96). Nous obtiendrons par là: $k_{1}=n^{\prime}, \varphi_{0}=0$ et $\alpha=0$. Ainsi le nombre $j_{n}$ des tours, produits autour de l'axe $O \xi$ par la courbe fermée $\Sigma$, sera donnée par

$$
j_{n}=J n+n^{\prime}
$$

Passons à présent à la question du nombre et de la position des points du croisement mutuel des parties de la trajectoire $\Sigma$. Si l'amplitude $\varphi$ est incommensurable avec $\pi$, le nombre de ces points est infiniment grand et ils couvrent entièrement par leurs positions la zone sphérique entre les circonférences $F_{2}$ et $F_{3}$. Mais si l'amplitude $\varphi$ est commensurable avec $\pi$, le nombre des points du croisement mutuel des moreeaux de la trajectoire $\Sigma$ sera fini. Pour éclaircir les propriétés de la trajectoire $\Sigma$, nous examinerons d'abord en détail les eroisements mutuels de ces morceaux pour le cas, où $\varphi$ et $\pi$ sont commensurables; puis au moyen des limites nous passerons au cas, où $\varphi$ et $\pi$ sont incommensurables.

Ainsi supposons que l'amplitude $\varphi$ est commensurable avec $x$ et peut être présentée par l'expression (96).

Les points $v_{m}$ de la circonférence $E$, figurant des quantités qui peuvent être déterminées pour toutes les quantités entières $m$ par l'expression (91), doivent, suivant la condition (96), se répéter périodiquement, en coïncidant arec $n$ de points

$$
v_{0}, v_{1}, v_{2}, \ldots, v_{n-1} \text {. }
$$

Ces points sont disposés sur la circonférence $E$ les uns après les antres non pas dans l'ordre de l'indice (à part le cas $n^{\prime}=1$ ), mais dans celui que nous étudierons plus à fond.

D'après la similitude de la répartition des points $Z$ et $z$, déterminés par les expressions (92) et liés par la reltion (68), la succession des points

$$
v_{0}, v_{1}, v_{2}, \ldots, v_{n-1}
$$

sur la circonférence $E$ doit être semblable à celle de la succession des points correspondants sur la circonférence $E_{0}$, points qui représentent les quantités 
où, en général,

$$
z_{0}, z_{1}, z_{2}, \ldots, z_{n-1}
$$

$$
z_{m}=e^{\frac{2 m n^{\prime} \pi i}{n}}
$$

Il s'ensuit de l'expression

$$
z_{m+1}=z_{m} e^{\frac{2 n^{\prime} \pi i}{n}}
$$

que $x^{\prime}-1$ points, pris du groupe:

$$
z_{0}, z_{1}, \ldots, z_{n-1}
$$

sont répartis sur l'arc $z_{m} z_{m+1}$, qui est décrite par le point $z=e^{\vartheta_{i}}$ pendant la croissance de $\vartheta$ de

$$
\frac{2 m n^{\prime} \pi}{\pi} \text { à } \frac{2(m+1) n^{\prime} \pi}{n} .
$$

Admettons que ces $n^{\prime}-1$ de points, étant disposés dans leur succession sur l'arc $z_{m} z_{m+1}$, soient présentés de la manière suivante:

$$
z^{\prime}, z^{\prime \prime}, \ldots, z^{\left(n^{\prime}-1\right)} \text {. }
$$

Il est évident que ces points représentent les quantités de la forme:

$$
z^{(k)}=z_{m} e^{\frac{2 k \pi i}{n}}
$$

où

$$
k=1,2, \ldots, n^{\prime}-1 \text {. }
$$

Admettons ensuite que les correspondants des points

seront:

$$
v_{0}, v_{1}, \ldots, v_{n+1}
$$

de sorte que

$$
v^{\prime}, v^{\prime \prime}, \ldots, v^{\left(x^{\prime}-1\right)}
$$

$$
v^{(k)}=P \tau \cdot \frac{W_{1}^{\prime} \tau+c z^{(k)} W_{2}^{\prime} \tau}{W_{1}^{\prime} \tau+c z^{(k)} W_{2} \tau} .
$$

En se basant sur l'analogie de la répartition des points $Z=v^{(k)}$ et des points $z=z^{(k)}$, les points

$$
v^{\prime}, v^{\prime \prime}, \ldots, v^{\left(n^{\prime}-1\right)}
$$

doivent être répartis sur l'arc $v_{m} v_{m+1}$ de la circonférence $E$ dans la succession des index. La répartition des points

$$
\nabla_{0}, \nabla_{1}, \ldots, \nabla_{n-1}
$$

sur la circonférence $F$ se soumet à la même loi, c. à. d. que les points

$$
V^{\prime}, V^{\prime \prime}, \ldots, V^{\left(n^{\prime}-1\right)}
$$


Sur un cas de rotation d'un solide autour d'un point fixe.

correspondant anx points

$$
v^{\prime}, v^{\prime \prime}, \ldots, v^{\left(x^{\prime}-1\right)}
$$

se répartissent sur l'are $V_{m} V_{m+1}$ de la eirconférence $F$ dans l'ordre de la croissance des index.

Après avoir fixé la répartition des points $V_{m}$ sur la circonférence $F$, passops à l'examen des morceanx

$$
\Sigma_{0}, \Sigma_{1}^{\prime}, \ldots, \Sigma_{n-1}
$$

de la trajectoire $\Sigma$, décrits par les points

$$
V_{0}, \nabla_{1}, \ldots, \nabla_{n-1}
$$

avec le changement de $\tau$ depuis - $\omega$ jusqu'à $+\omega$, Ces morceaux se composent d'une manière conforme premièrement des parties

$$
K_{q}, K_{1}, \ldots, K_{n-1},
$$

décrites par les points

$$
\nabla_{0}, \nabla_{1}, \ldots, \nabla_{n-1}
$$

pendant le changement du temps $\tau$ depuis $-\omega$ jusqu'à $Q$, et puis des parties

$$
L_{0}, L_{1}, \ldots, L_{22-1}
$$

décrites par les mêmes points pendant le changement de $\tau$ depuis 0 jusqu'à $+\infty$. Comme les points

$$
\nabla_{11}, \nabla_{1}, \ldots, \nabla_{n-1}
$$

ne peurent aueunement coïncider les uns avec les autres, les courbes

$$
K_{0}, K_{1}, \ldots, K_{n-1}
$$

ne peuvent évidemment pas se croiser; il en est de même des courbes

$$
L_{0}, L_{3}, \ldots, L_{n-1} \text {. }
$$

Cependant les courbes

$$
K_{0}, K_{1}, \ldots, K_{n-1}
$$

peuvent se croiser avec les courbes

$$
L_{0}, L_{1}, \ldots, L_{n-1} \text {. }
$$

Suivant la succession des points $\nabla_{m}$ sur la circonférence $F$, les courbes $K_{m}$ se disposent dans l'intérieur de la zone sphérique entre les circonférences $F_{2}$ et $F_{3}$ non pas dans l'ordre des index, mais dans un autre (excepté le cas $x^{\prime}=1$ ). D'après cet ordre les courbes

$$
K^{\prime}, K^{\prime \prime}, \ldots, K^{\left(n^{\prime}-1\right)}
$$

se disposent successivement entre les courbes $K_{m}$ et $K_{m+1}$. Les courbes

$$
K^{\prime}, K^{\prime \prime}, \ldots, \mathcal{K}^{\left(n^{\prime}-1\right)}
$$


sont décrites pendant le changement de $\tau$ depuis $-\omega$ jusqu'à $O$ par les points

$$
V^{\prime}, V^{\prime \prime}, \ldots, V^{\left(n^{\prime}-1\right)},
$$

qui sont disposés sur larc $\nabla_{m i} \nabla_{m+1}$ de la circonférence $F$.

Les conrbes $L_{m}$ se disposent dans la zone sphérique de la même manière, de sorte que les courbes

$$
L^{\prime}, L^{\prime \prime}, \ldots, L^{\left(n^{\prime}-1\right)},
$$

décrites pendant le changement de $\tau$ depuis 0 jusqu'à $\mathfrak{\omega}$ par les points

$$
\nabla^{\prime}, \nabla^{\prime \prime}, \ldots, \nabla^{\left(n^{\prime}-1\right)},
$$

se disposent entre les courbes $L_{m}$ et $L_{m+1}$.

Les courbes $L_{m}$ et $K_{m}$ forment par leurs croisements mutuels dans l'intérieur de la zone sphérique un filet, dont l'épaisseur déprend du nombre $j_{n}$ des tours, produits par la courbe fermée $\Sigma$ autour de l'axe $0 \xi$; le nombre $j_{n}$ est déterminé, comme nous l'avons vo, par l'expression (97). Si nous sousentendons par $j_{n}^{\prime}$ la valeur absolue du nombre $j_{n}$ désigné et par $M$ le nombre des points des croisements mutuels des morceaux de la trajectoire $\Sigma$, l'inégalité

$$
\mathcal{M} \geqq n\left(j_{n}^{*}-1\right)
$$

doit avoir lieu. On peut démontrer cette supposition comme il suit. Faisons passer par l'intérieur de la zone sphérique, que nous examinons, la courbe $l$ partant de la circonférence $F_{2}$ jusqu'àla eirconférence $F_{3}$. Admettons que la circonférence mobile $F$, sur laquelle les points $V_{m}$ sont disposés, croise, dans tontes ses positions, la courbe $l$ une seule fois. Nommons la courbe $l$, qui satisfait à toutes ces exigences, transversale de la zone sphérique. Distinguons par rapport à cette transversale deux rives, comme on distingue les rives sur le globe terrestre en dessinant la place de la rivière par une ligne courbe. Supposons ensuite, que le point $V$ décrive la trajectoire $\Sigma$, et examinons les passages du point $V$ par la transversale $l$, en les partageant en deux catégories, selon que le passage correspondant du point $V$ par la transversale $l$ se produit en partant de la rive droite et en allant à la rive gauche ou vice-versa. Les passages de chaque catégorie sont comptés séparément. La quantité absolue de la différence des deux nombres, que nous arons ainsi obtenus, doit coïncider avec le nombre absola $j_{n}^{\prime}$ des tours, produits par la courbe $\mathcal{E}$ autour de laxe O૬. En même temps il est évident que la courbe $\Sigma$ doit croiser la transversale $l$ moins de $j_{n}^{\prime}$ de fois. Supposons que la transversale $l$ est tracée de façon qu'elle n'ait avee la courbe $L_{m}$ qu'un senl point commun. Si avec cela nous excluons de la courbe $\Sigma$ la partie $L_{m}$, la transversale $l$ doit croiser la courbe $\mathcal{L}$ dans ses autres parties pas 
moins de $j_{n}^{\prime}-1$ de fois. Maintenant il est facile de voir, qu'en con* servant toutes les propriétés désignées de la transversale l nous pouvons la faire passer aussi près, que nous voulons, de la coïncidence avec la courbe $L_{m}$. Et c'est pourquoi la courbe $L_{m}$ doit se croiser avec les autres parties de la caurbe $\Sigma$ pas moins de $j_{n}^{\prime}-1$ de fais. Ce qui vient d'être dit, se rapporte à chacune des courbes $L_{0}, L_{1}, \ldots, L_{n-1}$. Par conséquent le nombre entier $M$ des points de croisement mutuel des morceaux de Ia trajectoire $\Sigma$ n'est pas plus pétit que le nombre $n\left(\hat{j}_{n}^{\prime}-1\right)$. Ainsi l'inégalité $(98)$ est démontrée.

L'inégalité (98) montre, que $n\left(j_{n}^{\prime}-1\right)$ est le minimum du nombre II des points du croisement mutuel des morceaux de la courbe $\Sigma$. Dans beaucoup de cas le nombre $M$ coïncide avec le minimum, c. à. d. qu'il est déterminé par l'expression:

$$
M=n\left(j_{n}^{\prime}-1\right) \text {. }
$$

Il en est ainsi par exemple dans les cas où l'amplitude $\phi$ de la quantité $p$, déterminée par l'expression (65̃), ou diminue constamment ou augmente constamment pendant la croissance du temps $\tau$. Mais dans certains cas l'amplitude désignée $\Phi$ balance, admettant avee quelquesunes des positions du point $v$ la croissance et avec d'autres la décroissance. Dans ces cas le nombre $M$ peut surpasser la limite inférieure $\left(j_{x}^{\prime}-1\right)$.

Si $\mathcal{J}=0$, on a d'après (97) $j_{n}^{\prime}=n^{\prime}$. Par conséquent, ayant $J=0$, l'inégalité (98) s'exprimera ainsi:

$$
M \geq n\left(n^{\prime}-1\right)
$$

et l'expression (99) qui détermine le minimum de $M$ devient:

$$
M=n\left(n^{\prime}-1\right) \text {. }
$$

On voit par l'inégalité (99') ce qui suit: quand le nombre $n^{\prime}$ est considérable, Ie nombre $M I$ des points de croisement mutuel des morceaux de la trajectoire $\Sigma$ doit être aussi considérable; donc l'épaisseur du filet formé par les croisements des courbes $K_{m}$ et $L_{m}$ doit être considérable.

Pour se faire une idée plus nette du filet, formé par la courbe $\Sigma$, fixons notre attention sur un cas plus simple, quand l'amplitude $\Phi$ de la quantité $v$ augmente ou diminue incessamment pendant la croissance du temps $\tau$. Ce cas ne peut pas avoir lien toujours, mais il peut se réaliser. Examinons dans ce cas non sealement le nombre, mais aussi la répartition relative des points du croisement mutuel des parties de la courbe $\Sigma$, en supposant qữe lindex $J$ est zẹ́ro, comme cela arrive tonjours, et que la direction de laxe $0 \xi$ est choisie de façon que $\Phi$ grandisse incessament. 
Aux conditions, que nous examinons, les morceanx successifs $\Sigma_{m}$ et $\Sigma_{m+1}$ de la courbe $\Sigma$ be peuvent pas se croiser. La courbe $\Sigma_{m}$, représentée schématiquement sur la figure 4 par la ligne $u u^{\prime} u_{1}$, et l'arc $u_{1} u$ de la circonférence $F_{3}$, renfermé entre les positions $u_{1}$ et $u$ des points $V_{m+1}$ et $V_{m}$ au moment $\tau=-\boldsymbol{\omega}$, pris ensemble

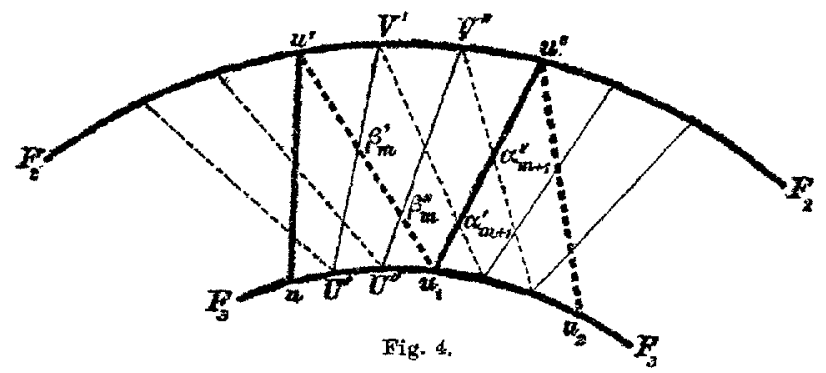

forment sur la sphère $O$ une courbe fermée $\sigma_{m}$, qui sépare une partie entière sur la surface de la sphère $O^{*}$ ). De même la courbe $\Sigma_{m+1}$, représentée sur la figure 4 par la ligne $u_{1} u^{\prime \prime} u_{2}$, et l'are $u_{2} u_{1}$ de la circonférence $F_{3}$, enfermé dans les positions $u_{2}$ et $u_{1}$ des points $V_{n+1}$ et $V_{m}$ au moment $\tau=+\omega$, forment sur la sphère $O$ une courbe fermée $\sigma_{m+1}$. En outre les parties $L_{m}$ et $K_{m+1}$ des courbes $\Sigma_{u k}$ et $\Sigma_{m+1}$, représentées sur la figure 4 par les lignes $u^{\prime} u_{1}$ et $u_{1} u^{\prime \prime}$, et l'arc $u^{\prime} u^{\prime \prime}$ de la eirconférence $F_{2}$, enfermé dans les positions $u^{\prime}$ et $u^{\prime \prime}$ des points $V_{m}$ et $V_{3 n+1}$ an moment $\tau=0$, forment ensemble sur la sphère $O$ une courbe fermée $u^{\prime} u^{\prime \prime} u_{1} u^{\prime}$, que nous désignerons par $\sigma_{m}^{\prime}$.

Les points ei-devant désignés

$$
\nabla^{\prime}, \nabla^{\prime \prime}, \ldots, \nabla^{\left(n^{\prime}-1\right)}
$$

qui se trourent sur l'are $V_{n} V_{m+1}$ de la circonférence $F$, se placent au moment $\tau=-\omega$ sur l'arc $u u_{1}$ de la circonférence $F_{3}$. Puis pendant le changement de $\tau$ depuis $-\omega$ jusqu'à 0 les points

$$
V^{\prime}, V^{\prime \prime}, \ldots, V^{\left(x^{\prime}-1\right)}
$$

décrivent, tout en gardant sur l'arc mobile $F$ la succession dans leur position, les parties

des morceanx

$$
K^{\prime}, K^{\prime \prime}, \ldots, K^{\left(x^{\prime}-1\right)}
$$

$$
\Sigma^{\prime}, \Sigma^{\prime \prime}, \ldots, \Sigma^{\left(n^{\prime}-1\right)}
$$

de la courbe $\Sigma$ et au moment $\tau=0$ ils se disposent sur l'are $u^{\prime} u^{\prime \prime}$ de la circonférence $F_{2}$. On voit par là que les points

$$
\nabla^{\prime}, \nabla^{\prime \prime}, \ldots, \nabla^{\left(n^{\prime}-1\right)}
$$

doivent se trouver au commencement de l'intervalle $\tau$ de - $\omega$ jusqu'à 0

*) Les points $u, u_{1}$ et $u_{2}$ ne présentent sur la figare 4 que la désignation plus briève des points $U_{m}, U_{m+1}$ et $U_{m+2}$ de la circonférence $F_{3}$. 
dans la surface, limitée par la courbe $\sigma_{m}$, eł vers la fin de ce même intervalle ils doivent se trouver en dehors de cette surface et au milieu de celle qui est limitée par la courbe $\sigma_{m}^{\prime} ;$ par conséquent ils doivent traverser la courbe $\sigma_{m}$ dans l'intervalle du temps $\psi$ de $-\infty$ jusqu'à 0 . Ce passage ne peut se produire que par l'arc $u^{\prime} u_{1}$, c. à. d. par la partie $L_{m}$ du morceau $\Sigma_{m}$, et non par l'are $u u^{\prime}$, parce que les courbes

$$
K, K^{\prime \prime}, \ldots, K^{\left(x^{\prime}-1\right)}
$$

ne peavent pas couper $u u^{\prime}$, c. à. d. la courbe $K_{m}$. Ainsi les parties

des morceaux:

$$
\mathcal{K}^{\prime}, \mathcal{K}^{\prime \prime}, \ldots, \mathcal{K}^{\left(n^{\prime}-1\right)}
$$

$$
\Sigma^{\prime}, \Sigma^{\prime \prime}, \ldots, \Sigma^{(i n-1)}
$$

doivent couper la partie $I_{m}$ du morceaux $\Sigma_{m}$ et chacune one seule fois (ce qui dérive de la croissance continuelle de la longitude $\Phi$ dn point $V$ ), Désignons ces croisements successives d'une manière conforme par $\beta_{m}^{\prime}, \beta_{m}^{\prime \prime}, \ldots, \beta_{m}^{\left(n^{\prime}-1\right)}$. Ces points doivent être placés d'après les circonstances, que nous examinons, le long de la courbe $L_{m}$ dans la succession des index supérieurs.

Supposons maintenant, que le temps $\tau$ change de 0 jusqu'à $+\omega_{*}$ Arec ces conditions les points

décrivent les parties

$$
V^{\prime}, V^{\prime \prime}, \ldots, V^{\left(n^{\prime}-1\right)}
$$

des morceaux

$$
L^{\prime}, L^{\prime \prime}, \ldots, L^{\left(x^{\prime}-1\right)}
$$$$
\Sigma^{\prime}, \Sigma^{\prime \prime}, \ldots, \Sigma^{\left(2^{\prime}-1\right)}
$$

et en méme temps ils passent du domaine, limité par la courbe $\sigma_{m}^{\prime}$, dans celui qui est limité par la courbe $\sigma_{\eta q+1}$, en se disposant dans le moment $\tau=\omega$ sur l'are $u_{1} u_{2}$. De cette façon les parties

$$
L, L^{\prime \prime}, \ldots, L^{\left(n^{\prime}-1\right)}
$$

des morceaux

$$
\Sigma^{\prime}, \Sigma^{\prime \prime}, \ldots, \Sigma^{\left(n^{\prime}-1\right)}
$$

conpent la courbe $u_{1} u^{\prime \prime}$ ou, autrement, la partie $K_{m+1}$ da morcean $\Sigma_{m+1}$ dans les points, que nous désignerons d'une manjère correspondante $\alpha_{m+1}^{\prime}, \alpha_{m+1}^{\prime \prime}, \ldots, \alpha_{m+1}^{\left(n^{\prime}-1\right)}$. Ces points se disposent le long de la courbe $K_{m+1}$ dans l'ordre de la décroissance des index supérieurs.

Ce qui vient d'être énoncé s'étend, si les conditions que nons étadions existent, sur tous les morceaux de la trajectoire du point $\nabla$ et montre que chaque morcean $\Sigma_{n}$ contient sur son étendne $2\left(n^{\prime}-1\right)$ de points, auxquels ce moreeau se croise avec ceux de la courbe $\Sigma$. La première partie de ces points de eroisement se trouve sur la partie $K_{m}$, et la seconde partie sur la partie $L_{m}$ de la courbe $\Sigma_{m}$. 
Sil se trouve sur chaque courbe $L_{m} n^{\prime}-1$ de points de croisement, produits par cette ligne avec les autres parties de la courbe $\Sigma$, le nombre entier $\boldsymbol{M}$ de tous les points de croisement mutuel des morceaux de la courbe $\Sigma$ s'exprimera, considérant les conditions nommées, de cette manière: $M=n\left(n^{\prime}-1\right)$ ce qui est entièrement conforme à la formule $\left(99^{\prime \prime}\right)$.

Passons à présent au cas où l'amplitude $\varphi$ de la quantité caractéristique $h$ est incommensurable avec le nombre $\pi$, de sorte que le rapport $\varphi: \pi$ est un nombre irrationnel. Pour se faire une idée des points de croisement mutuel de la trajectoire $\Sigma$ dans ce cas, changeons le nombre incommensurable $\varphi: \pi$ en un nombre rationnel rapproché et, tont en examinant ce cas rapproché, nous passerons ì la limite, quand ce nombre rationnel tend au nombre irrationnel désigné. Autrement dit, admettons avec un rapprochement l'existence de l'expression (96) avec laquelle le mouvement périodique des points $v$ et $V$ avec la période $2 n \omega$ aura de la valeur. Les nombres entiers $n$ ' et $n$ grandissent d'une manière illimitée par suite $d u$ rapprochement de la corrélation $n^{\prime}: n$ de la limite $\varphi: \pi$. Il se produit en même temps la croissance illimitée du période $2 n \omega$, ainsi que du nombre des croisements de chaque morceau $\Sigma_{m}$ arec les autres parties de la trajectoire $\Sigma$ du point $V$. Comme en même temps les points

$$
V^{\prime}, V^{\prime \prime}, \ldots, V^{\left(n^{\prime}-1\right)} \text {, }
$$

pris du nombre des points $V_{1}, \ldots, V_{n-1}$ et situés successivement sur l'are $V_{m} V_{m+1}$, avec la croissance des nombres $n$ ' et $n$ vont en se rapprochant et couvrent entièrement, dans la limite, l'arc $V_{m b} V_{m+1}$ de la circonférence $F$, les morceaux

$$
\Sigma_{0}, \Sigma_{j}, \ldots, \Sigma_{n-1}
$$

de la trajectoire $\Sigma$ couvrent entièrement, dans la limite, la zone sphérique entre les circonférences $F_{2}$ et $F_{3} ;$ les points de croisement des morceaux de la trajectoire $\Sigma$ remplissent aussi totalement cette zone sphérique. Autrement dit dans le cas donné la trajectoire $\boldsymbol{\Sigma}$ forme par le croisement réciproque de ses morceau un filet infiniment serré, c. à. d. un filet avec un nombre infini de points de sections, qui couvrent entièrement l'intérieur de la zone sphérique entre les circonférences $F_{2}$ et $F_{3}^{\prime}$.

Si dans le cas, que nous examinons, nous limitons la variation du temps $\tau$ par des limites finies, le point $V$ décrira dans cet intervalle fini une courbe finie, qui, se coupant elle-même, forme un filet avec un nombre fini de points de section; plus l'intervalle sera grand plus ce filet sera serré.

Nous remarquerons par rapport aux moments du temps $\tau$, pendant lesquels le point mobile $V$ passe par les points de section des morceanx 
de la trajectoire, qu'on peut se servir paur les déterminer de la même équation, qui se trouve daus le § précédent, a. à. d. de l'équation ( $\left.80^{\prime}\right)$.

Après avoir étudié la position relative des trajectoires du point $v$, correspondant à ses positions initiales différentes sur la circonférence $E_{2}$, nous remarquerons, qu'avec les conditions que nous examinons les racines $s_{1}$ et $s_{2}$ de l'équation (57), représentées par les expressions (60), et les quantités conjuguées sont liées par les corrélations:

$$
\bar{s}_{1} \bar{\delta}=-s_{2} \delta, \quad \bar{s}_{2} \bar{\delta}=-s_{1} \delta .
$$

A l'aide de ces correlations et des corrélations (53) nous nous convainquons que les quantités $q_{1}$ et $q_{2}$, déterminées par les expressions $\left(58^{\prime \prime}\right)$, et les quantités conjuguées remplissent les conditions:

$$
\bar{q}_{1}=q_{1}, \quad \bar{q}_{2}=q_{2},
$$

c. à. d. que les quantités $q_{1}$ et $q_{2}$ sont réelles. Par là et d'après less expressions $\left(58^{\prime \prime}\right)$ il s'ensuit que:

$$
W_{1}(-\tau)=\bar{W}_{+} \tau, \quad W_{2}(-\tau)=\bar{W}_{2} \tau .
$$

En différentiant ces correlations nous troupons:

$$
W_{1}^{\prime}(-\tau)=-\bar{W}_{1}^{\prime} \tau, \quad W_{2}^{\prime}(-\tau)=-\bar{W}_{2}^{\prime} \tau .
$$

Ayant ces correlations, nous allons examiner deux trajectoires du point $v$, pour lesquelles la position initiale (an moment $\tau=0$ ) coïncide avec l'un ou l'autre des points de l'intersection de la circonférence $E_{2}$ avec l'axe $O \xi$. Nous arons pour ces positions initiales; $v= \pm \sqrt{1-\xi_{2}^{2}}$. En déterminant par ces positions initiales les significations correspondantes $c^{\prime}$ et $c^{\prime \prime}$ de la constante $c$, nous obtenons:

$$
c^{r}=-\frac{q_{1}-\gamma_{2}}{q_{2}-\gamma_{2}}, \quad c^{\prime \prime}=-\frac{q_{1}+\gamma_{2}}{q_{2}+\gamma_{2}},
$$

où $\gamma_{2}$ est déterminé par l'expression ( $\left.86^{\circ}\right)$. Comme les deux mouvements que nous examinons se trouvent encore dans le cas général représenté au moyen de l'équation (93), nous arons: $c^{\prime}=c z^{\prime}, c^{\prime \prime}=c z^{\prime \prime}$ et par là $c^{\prime}=c^{\prime \prime} z$, où $z^{\prime}, z^{\prime \prime}$ et $z$ sont des quantités dont les modules coincident avec 1. Dans le cas présent la quartité $z$ doit coïncider avec -1 , car les quantités inégales $c^{\prime}$ et $c^{\prime \prime}$ sont réelles. Ainsi $z=-1$, autrement $c^{\prime}=-e^{\prime \prime}$. De là:

d'où il s'ensuit que:

$$
\frac{q_{1}+\gamma_{2}}{q_{2}+\gamma_{2}}=-\frac{q_{1}-\gamma_{2}}{q_{2}-\gamma_{2}}
$$

$$
q_{1} q_{2}=\gamma_{2}^{2} \text {. }
$$

C'est pourquoi nous pouvons supposer que:

$$
\left\{\begin{array}{l}
q_{1}=\gamma_{2} b, \\
q_{2}=\gamma_{2}: b .
\end{array}\right.
$$


Il est évident que la quantité $b$, faisant partie des l'expressions (101'), est réelle. Au moyen des expressions (101') nous nous convainquons que: $\left(101^{\prime \prime}\right)$ $c^{\prime}=-c^{\prime \prime}=b$.

Par conséquent les deux mouvements, auxquels le point $v$ se dispose sur l'axe $O \xi$ quand $\tau=0$, se déterminent à l'aide des expressions:

$$
v=P \tau \cdot \frac{W_{1}^{\prime} \tau \pm b W_{2}^{\prime} \tau}{W_{1} \tau \pm W_{2} \tau}
$$

où $b$ est une quantité, déterminée par les expressions $\left(101^{\prime}\right)$. Au moyen des expressions (100), (100') et $\left(100^{\prime \prime}\right)$ nous nous convajnquons que la quantité $v$, déterminée par l'expression (102), devient, avec le remplacement de $\tau$ par $-\tau$, la quantité conjuguée. Par conséquent la trajectoire de chacun des deux mouvements, que nous venons d'examiner, représente une courbe pour laquelle l'axe $O \xi$ est un axe de symétrie. Le point $v$ occupe sur chaque trajectoire les positions symétriques dans les moments $\tau$ et $-\tau$.

Dans l'expression (93) nous pouvons admettre: $c=b$, en sousentendant par $b$ une quantité déterminée par une des expressions $\left(101^{\prime}\right)$. Nous aurons par là:

$$
v=P \tau \cdot \frac{W_{1}^{\prime} \tau+b z W_{2}^{\prime} \tau}{W_{1} \tau+b z W_{2} \tau}
$$

où $z=e^{9 i}$. L'équation (103), coïncidant avec l'équation (65), concerne plus nettement une forme générale de la constante arbitraire $c$ dans la formule (65), précisément: $e=b e^{9 i}$, où $\vartheta$ est une quantite arbitraire réelle. Après avoir désigné la seconde partie de l'expression (103) par $v(\tau, z)$, nous nous convainquons an moyen des expressions $\left(100^{\prime}\right)$ et $\left(100^{\prime \prime}\right)$ que la fonction $v(-\tau, z)$ et la quantité conjuguée à la fonction

$$
v\left(\tau, \frac{1}{z}\right)
$$

coïncident. On voit par là que la trajectoire du point $v$, déterminée par l'expression (103), et la trajectoire du point $v$, déterminée par l'expression :

$$
v=P \tau \frac{W_{1}^{\prime} \tau+b \frac{1}{z} W_{2}^{\prime} \tau}{W_{1} \tau+b \frac{1}{z} W_{2} \tau},
$$

sont symétrique relativement à l'axe $O \xi$; le point $v$ occupe des positions symétriques sur l'une et l'autre des trajectoires dans les moments $\tau$ et $-\tau$.

Examinons parmi les mouvements du point $v$, déterminés par l'expression (103), deux mouvements, pour lesquels $z$ prend les valeurs suivantes:

$$
+e^{-\varphi i} \text { et }-e^{-\varphi i},
$$

où $\varphi$ est l'amplitude de la quantité caractéristique $h$. Il est facile de 
se convainere au moyen des expressions $\left(66^{\prime}\right),\left(100^{\prime}\right)$ et $\left(100^{\prime \prime}\right)$, que la quantité $v$ prend pour chacun de ces mouvements, quand $v=\ddot{\omega}+u$ et quand $\tau=\omega-u$, des valeurs conjuguées. Par conséquent chacune des trajectoires, que nous examinons, dn point $v$ présente une courbe, pour laquelle $O \xi$ est un axe de symétrie, arec lequel le point $v$ occupe sur la trajectoire des points symétriques aux moments $\tau=\omega+u$ et $\tau=\omega-u$. Chacune de ces deux trajectaires passe, comme il est facile de le voir, par un des points correspondant du croisement de la circonférence $B ;$ avec laxe $O \xi$ et le mobile $v$ passe par ce point au moment $\tau=a$.

Ainsi dans ce cas comme dans celui, que nous avons éxaminé dans le paragraphe précédent, nous n'arons que quatre trajectoires, pour lesquelles l'axe $O \xi$ est l'axe de symétrie.

Comme conclusion du paragraphe présent il faut dire, que le mouvement du point $v$, déterminé par l'expression (65), est instable à cause des perturbations qui agissent sur l'amplitude $\varphi$ de la quantité $h$, Effectivement, si l'amplitude $\varphi$ subit la moinure perturbation $\Delta \varphi$, la position du point $v_{m}$, déterminée par l'expression (91), et sa position agitée, qui représønte la quantité

$$
P \tau \cdot \frac{W_{1}^{\prime} \tau+c e^{2 m(\varphi+\Delta \varphi) i} W_{2}^{\prime} \tau}{W_{1} \tau+c e^{2 m(\varphi)+\Delta \varphi) i} W_{a}^{\prime} \tau}
$$

différent l'une de l'autre par une quantité finie, suivant le nombre entier $m$, pris à volonté; cette cireonstance oaractérise le mouvement instable du point $v$ dans le cas que nous examinons.

\section{$\S 8$.}

Mouvement du point $v$ daus le cas, où le solide est capable de reproduire un senl monvement asymptotique périodique avec la periode $2 \omega$.

Examinons ici le cas de transition, quand les racines des équations (57) sont égales $\left(s_{1}=s_{2}=s\right)$; la quantité caractéristique $h$ doit ètre égale à l'unité.

Dans ce cas le mouvement du point $v$ se détermine par l'expression (73) et sa position $v_{m}$ dans la phase du temps $\tau+2 m \omega$ est déterminée par l'expression (74). Si $m$ tend ̀̀ $+\infty$ ou à $-\infty$, $v_{z k}$ tend dans les deux cas à la rnême position limite

$$
v_{ \pm \infty}=P \tau \cdot \frac{W_{i}^{\prime} \tau}{W_{1} \tau}
$$

Par conséquent le moutement du point $v$, déterminé par l'expression (73), étant impériodique pour les changements finis du temps $\tau$, tend, arec la diminution infipie du temps $\tau$ ainsi qu'avec l'augmentation 
infinie, à un seul mouvement périodique avec la période $2 \omega$, déterminé au moyen de l'expression

$$
v=P \tau \cdot \frac{W_{i}^{i} \tau}{W_{1} \tau}
$$

Nommons ce mourement périodique mouvement asymptotique. C'est à ce mouvement unique asymptotique du cas donné qu'aboutissent les deux mouvements asymptotiques, déterminés par les expressions (75) et (76).

La liaison qui existe entre les quantités:

$$
Z=v_{n \mathrm{n}} \text { et } z=2 \mathrm{m \omega} \text {, }
$$

représentée par l'expression (74), appartient évidemment à la transformation linéaire

$$
Z=P \tau \cdot \frac{W_{1}^{\prime} \tau+c\left(W_{3}^{\prime} \tau+z W_{1}^{\prime} \tau\right)}{W_{1} \tau+c\left(W_{3} \tau+z W_{1} \tau\right)}
$$

Comme les tableaux des quantités $Z$ et $z$, déterminées par les ex. pressions (107), se disposent, ayant l'entière arbitraire $m$, l'une sur la circonférence $E$ et l'autre sur la droite $O \xi$, le point correspondant $Z$ doit changer constamment de place sur la circonférence $E$ pendant la translation continuelle de $z$ le long de l'axe $O \xi$.

On peut voir par là, en outre, que tous les mouvements mécaniques possibles du solide, que nous examinons, qui ne se diffèrent que par les positions initiales du point $v$ sur la circonférence $E_{2}$, sont déterminés, quand les autres circonstances initiales sont égales, par l'expression de la forme:

$$
v=P \tau \cdot \frac{W_{i}^{\prime} \tau+c\left(W_{3}^{\prime} \tau+z W_{1}^{\prime} \tau\right)}{W_{1} \tau+c\left(W_{3} \tau+z W_{2} \tau\right)},
$$

où $z$ est une quantité constante réelle.

Si z change de $-\infty$ jusqu'à $+\infty$, le point correspondant $Z$, déterminé par l'expression (108), décrit une seule fois la circonférence entière $E$ depuis le point $v_{ \pm \infty}$, représentant la quantité (105), jusqu'au retour à ce point. Par là on voit que les points

$$
v_{-\infty}, \ldots, v_{-2}, v_{-1}, v_{0}, v_{1}, v_{2}, \ldots, v_{+\infty},
$$

qui représentent les quantités $v_{m}$, déterminés par l'expression (74), se disposent le long de la circonférence $E$ dans l'ordre de la croissance des index. Cependant ils forment des deux côtés du point $v_{ \pm \infty}$ les lignes pointillées, dont les points contigus $v_{m}$ et $v_{m+1}$ se rapprochent infiniment à mesure qu'ils approchent de la position $v_{ \pm \infty}$. C'est de cette même manière que se disposent les points correspondants $V_{m}$ de la circonférence $F$ sur la sphère $O$.

Si nous supposons que le temps $\tau$ change de $-\omega$ jusqu'à $+\omega$, les points $\boldsymbol{V}_{m}$ décriront sur la sphère $O$ les courbes $\boldsymbol{\Sigma}_{m}$, qui forment ensemble une courbe continuelle $\Sigma$, par laquelle se mouve le point $V$. 
Les morceaux $\boldsymbol{\Sigma}_{m}$ de cette courbe se disposent successivement les uns après les autres dans la zone sphérique entre les circonférences $F_{2}$ et $F_{3}$. Dans la limite, quand $m$ tend on à $-\infty$ ou à $+\infty$, les morceaux $\Sigma_{m}$ tendent à une seule et même position de la limite $\Sigma_{ \pm(n)}$ qui représente en même temps la trajectoire fermée du point $V$ dans le cas du mouvement asymptotique périodique, détermipé par l'expression (106).

Prenant $m^{\prime}$ et $m^{\prime \prime}$ pour des nombres entiers, convenons de sousentendre par $\vartheta_{m^{\prime}} v_{m^{\prime \prime}}$ l'are de la circonférence $E$, décrit par le point $v$, qui représente une quantité, déterminée par l'expression (109), pendant le changement de $z$ de $2 m^{\prime} \omega$ jusqu'à $2 m^{\prime \prime} \omega$. Nous désignerons l'arc correspondant de la circonférence $F$ par $V_{m^{\prime}} V_{m^{\prime \prime}}$. Cet are ne forme qu'une partie de la circonférence $F$.

Désignons par $\sigma_{m}$ une courbe fermée, qui se compose de la courbe déjà nommée $\Sigma_{m}$ et de lare $U_{m+1} V_{m}$ de la circonférence $F_{3}$, avec lequel l'arc $V_{z u+1} V_{m}$ coïncide au moment $\tau=-\omega$. Les courbes ferméess

$$
\ldots, \sigma_{-2}, \sigma_{-1}, \sigma_{0}, \sigma_{1}, \sigma_{2}, \ldots
$$

doivent faire chacune un nombre égal de tours autour de l'axe $0 \xi$, ce qui est démontré par des procédés analogues à ceux, qui ont été employés dans les paragraphes précédentss et que pour plus de brièveté nous ne répéterons pas ici.

Nous désignerons par $J$ le nombre des tours, produits autour de l'axe $O \zeta$ par chacune des courbes $\sigma_{m n}$, et nous le nommerons «index» du mouvement, que nous examinons.

Dans le paragraphe suivant il nous sera démontré, que cet index est zéro. Maintenant bornons nous à remarquer: 1) que l'index $J$ garde sa constance arec le changement d'un mouvement ì un autre, pendant lequel change d'une manière arbitraire la position initiale du point $v$ sur la circonférence $E_{2}$; 2) que l'index $J$ garde sa constance arec le changement continuel des constantes $l_{1}, l_{2}$ et $a$, pendant lequel la quantité caractéristique $h$ reste tonjours égale à 1 . et quand les conditions (17) ont toujours lieu et $l_{1}$ ne devient jamais zéro. Cette constance est confirmée par des preuves analogues à celles qui se rencontrent dans les paragraphes précédents.

La courbe $\sigma_{m}$, que nous avons indiquée, change dans la limite en trajectoire $\Sigma_{ \pm \infty}$ du monvement asymptotique périodique, déterminé par l'expression (106), quand $m$ tend à $\pm \infty$. C'est pourquoi la courbe $\Sigma_{ \pm \infty}$ fait $J$ de tours autours de laxe $0 \xi$. Autrement dit, si le mouvement périodique (106) a lieu, la ligne verticale $O V$ fait dans le période du temps 2 a $J$ de tours autour de l'axe $0 \xi$. Comme en réalité $J=0$, ainsi que nous le verrons plus bas, la courbe $\Sigma_{ \pm \infty}$ ne fait aucun tour autour de l'axe $O \xi$ et par conséquent la droite $\overline{O V}$ ne peut pas non plus produire de tonrs autour da même axe. 
Admettons que le mouvement impériodique, déterminé par l'égalité (73), a lieu. Le nombre $j_{k}$ de tours, produits autour de l'axe $O \zeta$ par la partie de la trajectoire $\Sigma$, décrite par le point $V$ dans l'intervalle $2 \hbar \omega$, égal au nombre entier $\hbar$ des périodes $2 \omega$, se présente ainsi:

$$
j_{k}=J \cdot k+\alpha,
$$

où $-1<\alpha<+1$. La démonstration de l'expression (110) est analogue aux démonstration des formules (82) et (95) des paragraples précédents et n'exige d'être répétée. Remarquons ce qui suit: si nous exprimons le moment, d'où commence l'ỉntervalle $2 \%$, que nous examinons, de la façon suivante: $\tau+2 \hbar \omega$ où $-\omega \leqq \tau \leqq+\infty$, le nombre $\alpha$, faisant partie de l'expression (110), présente le nombre de tours, produits autour de l'axe $O \xi$ par l'are $V_{m} V_{m+k}$ de la circonférence $F$. Si $k$ est un nombre fini et si $m$ tend à $\pm \infty$, l'are $V_{m} V_{m+k}$ tend à zéro et le nombre correspondant $\alpha$ dans l'expression (110) tend aussi à zéro.

Si $J=0$, le nombre $j_{k}$ des tours, produits autour de l'axe $O \xi$ par la route, décrite par le point $V$ dans l'intervalle $2 k \omega$, est déterminé par l'expression: $j_{k}=\alpha$ où $-1<\alpha<+1$. Ce nombre $j_{k}$ reste fini avec le nombre $k$ aussi grand que l'on veut, et à cause de cela l'amplitude $\Phi$ de la quantité $v$, déterminée par l'expression (73), doit toujours varier, avec la croissance illimitée du temps $\tau$, dans les limites finies et doit rester finie, quand $\tau=\infty$.

Pour être plus brefs nous ne ferons pas de détails sur les croisements réciproques des morceaux de la trajectoire $\Sigma$, croisements qui possèdent des qualités analogues à celles, que nous avons éxaminées dans le $\$ 6$. Nous ne remarquerons que ce qui suit. Les morceaux de la trajectoire $\Sigma$ forment par leurs croisements réciproques dans l'intérieur de la zone sphérique, renfermée entre les circonférences $F_{2}$ et $F_{3}$, un filet, qui atteint sa plus grande épaisseur près du mourceau $\Sigma_{\Psi_{\infty}}$ et surtot près des points de ce morceau, qui se trouvent sur les circonférences $F_{2}$ et $F_{3}$.

Passons à présent à l'examen de la position relative de la trajectoire $\Sigma$ avec différentes circonstances.

Au moyen des expressions $\left(7 \mathscr{Y}^{\prime}\right),\left(72^{\prime \prime}\right)$ et $(53)$ nous nous convainquons que

$$
\bar{q}_{1}=q_{1}, \quad \bar{q}_{3}=-q_{3},
$$

Il en résulte de là et des expressions $\left(58^{\prime}\right)$ et $\left(71^{\prime}\right)$ que

$$
W_{1}(-\tau)=\bar{W}_{1} \tau, \quad W_{3}(-\tau)=-\bar{W}_{3} \tau \text {. }
$$

En différentiant ces expressions, nous trouvons:

$$
W_{1}^{\prime}(-\tau)=-\bar{W}_{1}^{\prime} \tau, \quad W_{3}^{\prime}(-\tau)=\bar{W}_{3}^{\prime} \tau .
$$

Les corrélations $(111)$ et $\left(111^{\prime}\right)$ montrent, que la quantité $v$, déterminée par l'expression (106), change en une quantité conjuguée 
avec le changement de $\tau$ en $-\tau$. Par conséquent dans le cas du mouvement asymptotique périodique du point $v$ sa trajectoire $S_{ \pm \infty}$ présente une courbe, pour laquelle la droite $O \xi$ est un axe de symétrie, et le point mobile $v$ occupe des positions symétriques dans les moments $\tau$ et $-\tau$ et arrive sur l'axe $O \xi$ dans les moments $\tau=0$ et $\tau=a$. En même temps cette trajectoire doit passer par un des points de l'intersection de la circonférence $E_{2}$ avec l'axe $O \xi$, et aussi par un des points d'intersection de la circonférence $E_{3}$ avec le même axe. Pour couper la trajectoire $S_{ \pm \infty}$ avec l'axe $O \xi$ qui se trouve sur la circonférence $E_{2}$, nous avons: $r=0$ et

$$
v=P 0 \cdot \frac{W_{1}^{\prime} 0}{W_{1} 0}=P 0 \cdot q_{1} \ddot{u}=b \sqrt{1-\xi_{2}{ }^{2}},
$$

où $b= \pm 1$. Le signe de la quantité $b$ est déterminé entièrement par: l'expression (112), qui est reduite à la forme:

$$
q_{1}=b \gamma_{2}
$$

où $\gamma_{z}$ est déterminé par l'expression $\left(86^{\prime}\right)$.

Déterminons la trajectoire du point $v$, qui passe par un autre point d'intersection de la circonférence $E_{2}$ avec l'axe $O \xi$, c. à. d. par le point, pour lequel, ayant $\tau=0$, nous arons:

$$
v=-b \sqrt{1-\xi_{2}^{2}} \text {. }
$$

Supposant dans l'expression (73) $\tau=0$, nous trouvons pour ce point:

$$
-b \sqrt{1-\xi_{2}^{2}}=P 0 \cdot i\left(q_{1}+c q_{3}\right)
$$

d'où nous obtenons en considérant l'expression (112):

$$
c=-\frac{2 q_{1}}{q_{3}}=\beta i \text {. }
$$

Au moyen des expressions (110') nous nous convainquons que la quantité $\beta$, déterminée par l'expression (115), est réelle. Le mouvement du point $v$, pour lequel l'expression (114) a lieu quand $\tau=0$, est déterminé par l'équation:

$$
v=P \tau \cdot \frac{W_{1}^{\prime} \tau+\beta i W_{3}^{\prime} \tau}{W_{1} \tau+\beta i W_{3} \tau} .
$$

Cette quantité $v$ devient quantité conjuguée quand $\tau$ est remplacé par - $\tau$. Par conséquent la trajectoire, que nous examinons, présente une courbe, pour laquelle l'axe $O \xi$ est l'axe de symétrie. Le point mobile $v$ occupe des positions symétriques sur cette trajectoire dans les moments $\tau$ et $-\tau$.

Dans l'expression (109) nous pouvons introduire à la place de $c$ une quantité, déterminée par l'expression (115). Nous aurons alors:

$$
v=P \tau \frac{W_{1}^{\prime} \tau+\beta i\left(W_{3}^{\prime} \tau+z W_{1}^{\prime} \tau\right)}{W_{1} \tau+\beta i\left(W_{3} \tau+z W_{1} \tau\right)}
$$

où $z$ est une quantité constante arbitraire réelle. L'équation (117) 
coïncidant avec l'équation (73), concerne plus nettement une forme générale de la constante arbitraire $c$ dans la formule (73), précisément: $c=\beta i:(1+z \beta i)$. Ayant désigné la deuxième partie de l'expression (117) par $v(\tau, z)$, nous nous convainquons au moyen des expressions (111) et $\left(111^{\prime}\right)$, que la fonction $v(-\tau, z)$ et la quantité, conjuguée à la fonction $v(\tau,-z)$, coïncident. Il s'ensuit que les trajectoires des deux mouvements du point $v$, déterminés par l'expression (117) avec deux valeurs de ż, égales par la quantité et différentes par les signes, sont disposées symétriequement par rapport à l'axe $O \xi$, pendant quoi le point $v$ occupe sur l'une et l'autre des trajectoires des positions symétriques dans les moments $\tau$ et $-\tau$.

Examinons ensuite la trajectoire da mouvement, déterminée par l'expression (117) ayant $z=-\omega$. Il est facile de voir au moyen des expressions $(70),\left(73^{\prime}\right),(111)$ et $\left(111^{\prime}\right)$, que la quantité $v$, ayant $\tau=\omega+u$ et $\tau=\omega-u$, prend des valeurs conjuguées. Par conséquent la trajectoire, que nous examinons, présente une courbe, pour laquelle l'axe $0 \xi$ est un axe de symétrie. Cette trajectoire doit passer par l'un des points d'intersection de l'axe $O \xi$ avec la circonférence $E_{3}$, et justement par celui des deux points par lequel ne passe pas la courbe $S_{\underline{i} \infty}$.

Ainsi dans le cas présent il existe non pas quatre trajectoires pour lesquelles $l^{\prime} O \xi$ est un axe de symétrie (comme dans les cas, examinés dans les deux paragraphes précédents), mais sealement trois.

Par le choix de la position initiale du point $v$ on peut obtenir que le mouvement asymptotique périodique (106) se réalise immédiatement. Ce mouvement, obtenu au moyen de celui de (117), quand $z= \pm \infty$, n'est pas évidemment stable si la position initiale du point $v$ sur la circonférence $E_{2}$ est agitée et si les autres circonstances initiales du mouvement sont restées sans changements.

\section{$\S 9$.}

Coïnoidence de l'index $J$ du mouvement avec zéro. Signes simplifiés de l'existence des mouvements asymtotiques périodiques.

Nous arons établi ci dévant l'idée de l'index $\mathcal{J}$ pour tous les cas possibles du mouvement, n'écartant que le cas où $l_{1}=0$. En même temps nous arons préparé dans l'énonciation précédente tontes les bases nécessaires pour la preuve du théorême, par lequel cet index $J$ égale toujours zéro. Passons maintenant à la démonstration même de ce théorême.

Nous avons établi séparément dans chacun des trois cas, qui dépendent des propriétés de la quantité caractéristique $h$, que le nombre $J$ ne change pas arec les changements continuels des quantités $l_{1}, l_{2}$ 
et $a$, si certaines conditions sont observées. Nous unirons cette conclusion, en démontrant, que la constance de l'index $J$ a lieu indépendamment de la valeur que prend la quantité caractéristique $h_{\text {, à }}$ condition que les inégalités (17) soient remplies et que la quantité $l_{1}$ ne se change pas en zéra pendant les changements continuels des quantités $l_{1}, l_{2}$ et $a$. La démonstration de cette thèse se rednit, en réalisant les conditions ennoncées, à la démonstration des deux propositions suivantes:

I. Si la quantité caractéristique $h$, qui change continuellement partant de la valear réelle, qui remplit la condition (62), tend en cass limite à 1 , lindex $J$ du mouvement lipite, ayant $h=1$, garde la valeur qu'il ayait avec les valeurs réelles $h$, contiguës à 1 .

II. Si la quantitế caractéristique $h$, qui change continuellement partant de la valeur imaginaire, satisfaisante les conditions (63), tend en cas limite à 1 , l'index $J$ du mouvement limite, ayant $h=1$, garde la même valeur qu'il avait avee les valeurs imaginaires $h$, contiguës à 1 .

Si la quantité $h$, étant réelle, tend continuellement à 1 , les deux mouvements asymptotiques périodiques (75) et (76) coincident, en cas limite, en un seul mouvement (106), avec quof les trajectoires fermées correspondantes $\Sigma_{-\infty}$ et $\Sigma_{+\infty}$, examinées dans le $\$ 6$, en se déformant sans la rupture de leurs parties et sans le passage par l'axe $0 \xi$, coïncident avec la trajectoire fermée $\Sigma_{ \pm \infty}$, que nous avons éxaminée dans le $\$ 8$ et qui est décrite par le point $V$ dans le mouvement asymptotique périodique (106). Par conséquent les courbes fermées $\Sigma_{-\infty}$ et $\boldsymbol{\Sigma}_{+\infty}$, ayant les valeurs réelles de $h$ contiguës arec 1 , et la courbe fermée $\Sigma_{ \pm \infty}$, avec $h=1$, font chacune le mêrne nombre de tours autour de l'axe $O \xi$ et déterminent ainsi la mềme valeur de l'index $J$. La première des deux positions est démontréé.

Si la quantité $h$ est imaginaire, il ne peut pas exister un seal des mouvements (75) et (76) dans le solide. C'est pourquoi nous emploierons un autre procédé, quand $h$ est imaginaire. Prenous un mouvement déterminé par l'équation (65) ou mienx, par l'équation équivalente (103), qui, comme il est facile de s'en convaincre an moyen des expressions $\left(58^{\prime}\right)$, peut être représenté par la forme suivante:

$$
v=P_{\tau} \cdot \frac{(1+b z) W_{1}^{\prime} \tau-b z\left(q_{1}-q_{2}\right) w_{2}^{\prime} \tau}{\left.(1+b)^{\prime}\right) W_{1} \tau-b_{2}\left(q_{1}-q_{2}\right) w_{2} \tau}
$$

où $z=e^{\vartheta i}$ et où $\vartheta$ est ane constante arbitraire réelle. Admettons que la quantité $z$ différe de \pm 1 par une quantité finie. Représentons la courbe $\sigma_{0}$ fermée, prise du nombre des conrbes $\left(94^{\prime}\right)$ et composée de la courbe $\Sigma_{0}$ et de l'arc $U_{1} U_{0}$ de la circonférence $F_{3}^{\prime}$. Passons à la limite quand la quantité $h$ tend à 1 et quand, par conséquent, l'amplitude $q$ tend à zéro. Dans la limite la différence $q_{1}-q_{2}$ tend 
à zéro; la quantité $b$, déterminée par les expressions (101'), tend on $\grave{a}+1$ ou à -1 ; la quantité $1+b z$ tend à la quantité $1 \pm z$ finie et différente de zéro; la deuxième partie de l'expression (118) tend, pour les valeurs finies de $x$, à la coïncidence avec la deuxième partie de l'expression (106); le morceau $\Sigma_{0}$, décrit par le point $V$ de la sphère $O$ dans l'intervalle fini du temps (de $\tau=-\omega$ jusqu'à $\tau=+\infty$ ), tend à la coïncidence avec la trajectoire fermée $\Sigma_{ \pm \infty}$ du mouvement asymptotique périodique (106), qui a lieu quand $h=1$; lare $U_{0} U_{1}$ de la circonférence $F_{3}$, qui fait partie de la courbe fermée $\sigma_{0}$, tend à zéro [nous nous en convainquons, en déterminant par la formule (94) le nombre $j$ des tours, produits par l'are $U_{0} U_{1}$ autour de l'axe $0 \xi$. Arec ces circonstances la courbe fermée $\sigma_{0}$ doit tendre à la coïncidence avec la courbe ci-devant désignée $\Sigma_{ \pm \infty}$, en même temps cette déformation de la courbe $\sigma_{0}$ s'opère continuellement, c. à. d. sans la rupture de ces parties et sans leur passage par l'axe $O \zeta$. Par conséquent la courbe $\sigma_{0}$ et la courbe $\Sigma_{ \pm \infty}$, avec laquelle cö̈ncide $\sigma_{0}$ quand $h=1$, font le même nombre de tours autour de l'axe $O \xi$, ce nombre détermine une seule et unique valeur de l'index $J$. Ainsi la deuxième supposition est démontrée.

Si la constance de l'index $J$ se conserve dans la limite, quand $h$ tend d'une manière ou d'une autre à 1 , elle doit se conserver avec le passage continuel de $h$ des valeurs réelles aux valeurs imaginaires et vice-versa, puisque ce passage n'est possible pour la quantité caractéristique $h$ que par $h=1$. En plus la constance de l'index $J$, pendant les autres changements continuels $l_{1}, l_{2}$ et $a$, est déjà établie dans les paragraphes précédentes. C'est pourquoi la constance de l'index $J$ pendant les changements, que nous examinons, est démontrée en général, indépendamment de la valeur $h$.

Si, par conséquent, il est possible de produire un passage continuel d'un système des quantités $l_{1}, l_{2}$ et $a$ à un autre en conservant pendant tout le temps du passage les conditions (17) et si en même temps $l_{1}$ reste toujours distinct de zéro, nous pouvons affirmer, malgrè la quantité $h$, que lindex $J$ du mouvement pour l'an et pour l'autre système des quantités constantes $l_{1}, l_{2}$ et $a$ est le même.

Supposons que le système donné des quantités $l_{1}, l_{2}$ et $a$ satisfasse les conditions $(17)$ *) et admettons que $l_{1}$ est différent de zéro. Changeons continuellement la quantité $a$ jusqu'à zéro, en laissant intact les quantités $l_{1}$ et $l_{2}$. On obtient ce changement de la quantité $a$, comme on le voit d'après l'expression (13'), par le rapprochement d'une des quantités $A$ et $C$, qui n'entrent nullement dans les conditions (17), à la

*) La quantité a ne rentre pas immédiatement dans les conditions (17); mais il $y$ entre le moment $\boldsymbol{B}$ dont depend la quantité $a$. 
quantité $B$. Ce changement ne peut pas enfreindre les conditions (17) et ne peut pas non plus rompre la constance de l'index $J$. Si, par conséquent, l'index $J$ est zéro avec $a=0$, il est en général zéro. Arretons done notre attention sur la détermination de l'index $J$ dans le cas où $a=0$.

Dans ce cas l'équation (42) prend la forme:

$$
\frac{d^{2} w}{d \tau^{2}}-\frac{1}{2}\left(\frac{\xi^{\prime} T-e_{0}^{\prime}}{\partial T-e_{0}}\right) \frac{d w}{d \tau}=0 .
$$

Les résolutions fondamentales particulières de ces équations seront

$$
w_{\mathrm{i}} \tau=1, \quad w_{2} \tau=i \int_{0}^{\tau} \sqrt{\frac{e_{0}-\xi T}{e_{0}-e_{2}}} \cdot e^{i x \tau} d \tau,
$$

où $\chi \tau$ est une fonction, déterminée par l'expression $\left(53^{\prime \prime}\right)$. Les coefficients $x, \lambda, \mu$ et $\nu$, déterminés par les expressions (52) at $\left(53^{\prime \prime \prime}\right)$, sexont exprimés ainsi:

$$
x=1, \quad \lambda=0, \quad \mu=2 i e^{i \chi^{\omega} \omega} \mu_{1}, \quad \nu=e^{2 i \chi^{\omega}},
$$

où

$$
\mu_{1}=\int_{0}^{\omega} \sqrt{\frac{e_{0}-\beta^{\alpha}}{e_{0}-e_{2}}} \cdot \cos (\chi \omega-\chi \tau) d \tau .
$$

La quantité $N$, déterminée par l'expression $\left(61^{\prime}\right)$, sera représentée ainsi:

$$
N=\cos x^{\omega} \text {. }
$$

En même temps la quantité caractéristique $h_{b}$ sera imaginaire et sera exprimée par l'une des quatre quantités:

$$
\pm e^{ \pm i \chi \omega}
$$

On doit choisir parmi ces quatre quantités celle dont l'amplitude $\varphi$ satisfait les conditions (63). Prenant la quantité $l_{1}$ pour positive [ce qui ne borne pas la généralité du raisonnement, car le remplacement de $\tau$ par $-\tau$, qui occasionne dans l'équation (42) le remplacement de $l_{1}$ par $-l_{1}$, est toujours possiblel, naus ferons remarquer qu'en même temps $\chi \omega$ sera aussi positive. Puis démontrons que l'amplitude $\varphi$, que nous cherchons, de la quantité caractéristique $h$ sera:

$$
\varphi=x \omega
$$

de sorte que

$$
h=e^{i \chi^{\omega}} .
$$

Pour se convaincre de l'exactitude de cette conclusion, il suffit de montrer que la quantité positive $\chi^{\omega}$ satisfait l'inégalité:

$$
x \omega<\frac{\pi}{2} .
$$


Nous arons:

$$
\chi \omega=\frac{l_{1}}{2} \int_{0}^{\omega} \frac{d \tau}{\bar{e}_{0}-\xi T}=\frac{l_{1}}{2} \sqrt[3]{\frac{2}{\bar{B}_{0}}} \int_{0}^{\omega} \frac{d \tau}{\xi-\xi_{0}} .
$$

Après avoir remarqué, que:

$$
\frac{d \xi}{d \tau}=\sqrt[3]{\frac{2}{B \varrho_{0}}} \cdot R=\sqrt[3]{\frac{2}{B \varrho_{0}}} \sqrt{2 B \varrho_{0}\left(1-\zeta^{2}\right)\left(\xi-\xi_{0}\right)-l_{1}^{2}}
$$

et en remplaçant dans l'intégrale précédent le variable $\tau$ par $\zeta$, nous trouvons:

$$
\begin{gathered}
\chi \omega=\frac{l_{1}}{2} \int_{\xi_{2}}^{\xi_{3}} \frac{d \zeta}{\left(\xi-\xi_{0}\right) A} \\
=\frac{l_{1}}{2 \sqrt{2 B Q_{0}}} \int_{\xi_{2}}^{\zeta_{3}} \frac{d \xi}{\left(\xi-\xi_{0}\right) \sqrt{\left(\xi-\zeta_{1}\right)\left(\xi-\xi_{2}\right)\left(\zeta_{3}-\bar{\zeta}\right)}} .
\end{gathered}
$$

Sousentendant par $;$ une valeur moyenne de la variable $\xi$, qui varie entre les limites de l'intégration:

$$
\xi_{2}<z<\xi_{3},
$$

nous arons

$$
\begin{gathered}
\int_{\xi_{2}}^{\xi_{3}} \frac{d \xi}{\left(\xi-\xi_{0}\right) \sqrt{\left(\xi-\xi_{1}\right)\left(\xi-\xi_{2}\right)\left(\xi_{3}-\bar{\zeta}\right)}} \\
=\frac{1}{\sqrt{\delta}-\xi_{1}} \int_{\xi_{2}}^{\xi_{\xi}} \frac{d \xi}{\left(\xi-\xi_{0}\right) \sqrt{\left(\bar{\zeta}-\xi_{2}\right)\left(\xi_{z}-\bar{\zeta}\right)}}=\frac{\pi}{\sqrt{\left(\xi-\xi_{1}\right)\left(\xi_{2}-\xi_{0}\right)\left(\xi_{3}-\overline{\left.\xi_{0}\right)}\right.}} .
\end{gathered}
$$

On voit par cette expression et par l'expression

$$
\frac{l_{1}}{\sqrt{2 \bar{B} e_{0}}}=\sqrt{\left(1-\xi_{2}^{2}\right)\left(\xi_{2}-\xi_{0}\right)}
$$

que la quantité $\chi \omega$ peut être exprimée ainsi:

$$
\chi \omega=\frac{\pi}{2} \sqrt{\left(\frac{1+\xi_{2}}{\delta-\xi_{1}}\right) \cdot\left(\frac{1-\xi_{2}}{\xi_{3}-\xi_{0}}\right)} .
$$

En même temps la première des inégalités (19) et la première des inégalités (121') démontrent que

$$
0<\frac{1+\xi_{2}}{z-\xi_{1}}<1 .
$$

En même texps la première des inégalités (19) et l'équation

démontrent, que

$$
\xi_{1}+\xi_{2}+\xi_{3}=\xi_{0}
$$

$$
0<\frac{1-\xi_{2}}{\xi_{3}-\xi_{0}}<1 \text {. }
$$


L'équation (122) et les inégalités (122') et (122') affirment entièrement l'exactitude des inégalités (121). Par conséquent l'amplitude $\varphi$ de la quantité caractéristique $h$, satisfaisant les conditions (63), se détermine par l'équation $\left(1200^{\circ}\right.$.

Après avoir déterminé la quantité caractéristique $h$ et son amplitude $\varphi$, nous trouvons au moyen de $\left(119^{\prime \prime}\right),(60)$ et $\left(58^{\prime \prime}\right)$

$$
s_{1}=1, \quad s_{2}=e^{2 i \chi \omega}, \quad q_{1}=0, \quad q_{2}=\frac{\sin \chi^{\omega}}{\mu_{1}},
$$

avec quoi les résolutions canoniques $W_{1} \tau$ et $W_{2} \tau$ doivent être exprinées ainsi:

$$
W_{1} \tau=1, \quad W_{2} \tau=1+q_{2} i \int_{0}^{\tau} \sqrt{\frac{e_{0}-\sigma T}{e_{0}-c_{2}}} e^{i x^{\tau}} d \tau .
$$

En déterminant ensuite $v$, nous remarquons que les expressions (65) et (93) exigent pour passer à la limite, quand a derient zéro, des préparations préliminaires. La constante $c$, qui se trouve dans l'expression (65) et qui est déterminée d'après la position initiale de $v$ sur la circonférence $E_{2}$, dépend de $a$. Il est nécessaire d'examiner cette dépendance. Les résolutions canoniques $W_{1} \tau$ et $W_{2} \tau$ de l'expression (42) peuvent être développées, avant de passer à la limite, par les degrés $a^{2}$, avee quoi les deuxièmes parties des équations (123) ne présenteront que les premiers membres de ces développements. Par conséquent, avant de passer à la limite, les résolutions $W_{1} \tau$ et $W_{2} \tau$ seront exprimées ainsi:

$$
\left\{\begin{array}{l}
W_{1} \tau=1+a^{2} i f_{1} \tau, \\
W_{2} \tau=1+q_{z} i\left[\int \frac{e_{0}-\overline{e_{j}} \bar{T}}{e_{0}-e_{2}} \cdot e^{i \chi^{\tau}} d \tau+a^{2} f_{2} \tau\right],
\end{array}\right.
$$

où $f_{1} \tau$ et $f_{2} \tau$ sont des fonctions qui gardent des valeurs finies ayant $a=0$ et qui changent en zéro quand $\tau=0$. Supposant qu'avec $\tau=0$ le point $u$ occupe la position initiale, déterminée par l'expression

$$
v=\sqrt{L-\xi_{z}^{2}} \cdot e^{\beta i}
$$

introduisous les expressions (123') dans l'équation (65́) et examinons cette équation ayant $\tau=0$. Nous trouverons

$$
a \sqrt{e_{0}}-e_{2} \cdot e^{\beta i}=\frac{a^{8} f_{1}^{\prime} 0+c q_{2}\left(1+a^{2} f_{2}^{\prime} 0\right)}{1+c}
$$

Il s'ensuit que

$$
c=\frac{a \sqrt{e_{0}-e_{2}} \cdot e^{\beta i}}{q_{2}}(1+a \varepsilon),
$$

où $\varepsilon$ est une quantité qui garde une valeur finie arec $a=0$. 
Après avoir introduit dans l'équation (65) les quantités $W_{1} \tau, W_{2} \tau$ et $c$, déterminées par les expressions $\left(123^{\prime}\right)$ et (124), puis en abrégeant dans l'expression $v$, que nous avons obtenue, le multiplicateur $a$ dans le nominateur et dans le dénominateur et en passant enfin à la limite, quand $a=0$, nous trourons:

où

$$
v=c_{1} \cdot \frac{\gamma^{\prime} T-e_{0}^{\prime}}{\sqrt{e_{0}-\wp T}} \cdot e^{i \chi \tau}
$$

$$
c_{1}=\frac{i e^{\beta i}}{2} \sqrt[3]{\frac{2}{B_{\varrho_{0}}}}
$$

De même l'expression (93) prend, en passant à la limite quand $a=0$, la forme:

$$
v=c_{1} \cdot \frac{\varphi^{\prime} T-e_{0}^{\prime}}{\sqrt{e_{0}-\varphi T}} e^{i(\vartheta+x \tau)}
$$

Maintenant nous passons à l'éxamen du nombre $J$ des tours, produits antour de l'axe $O \xi$ par la courbe fermée $\sigma_{0}$, composée du morcean $\Sigma_{0}$ de la trajectoire $\Sigma$ et de l'are $U_{1} U_{0}$ de la circonférence $F_{3}$ (supposant $a=0$ ).

Le morceau $\Sigma_{0}$ est décrit par le point $V$, représentant la quantité $v$, déterminée par l'expression (125), pendant que $\tau$ change de $-\omega$ jusqu'à $+\omega$. Dans cet intervalle l'amplitude de la quantité:

$$
c_{1} \frac{\beta^{\prime} T-e_{0}^{\prime}}{\sqrt{e_{0}-p^{\prime} T}}
$$

recevra un accroissement égal à zéro; tandis que l'amplitude de la quantité $e^{i \chi \tau}$ recevra un accroissement égal à

$$
\chi \omega-\chi(-\omega)=2 \chi \omega \text {. }
$$

Cet accroissement coïncide à celui de l'amplitude $\Phi$ de la quantité $v$ qui est obtenu pendant que le point correspondant $V$ décrit l'arc $\Sigma_{0}$.

L'are $V_{0} V_{1}$ de la circonférence $F$ est décrit, avec la constante $\tau$ et avec la croissance de $\vartheta$ de 0 jusqu'à $2 \varphi=2 \chi^{\omega}$, par le point $V$ qui représente la quantité $v$, déterminée par l'expression $\left\langle 125^{\prime}\right)$. Pendant ce déplacement du point $V$, l'amplitude $\Phi$ de la quantité correspondante $v$ reçoit l'accroissement de $2 \chi \omega$. Cet accroissement de l'amplitude $\Phi$ correspond aussi à l'arc $U_{0} U_{1}$, avec lequel coüncide l'are $\nabla_{0} V_{1}$ au moment $\tau=-\omega$. Quant à l'accroissement, qui correspond à l'arc $U_{0} U_{1}$ passé en sens invers, e. à. d. à l'arc $U_{1} U_{0}$, il s'exprimera ainsi:

$$
-2 \chi \omega \text {. }
$$

En additionnant les accroissement ci-devant nommés de l'amplitude $\Phi$, lesquels correspondent au passage des arcs $\Sigma_{0}$ et $U_{1} U_{0}$, nous obtenons zéro. Ce zéro représentera l'accroissement de l'amplitade $\Phi$ de la quantité $v$, obtenue pendant que le point correspondant $V$ décrit 
la courbe fermée $\sigma_{0}$. Par conséquent le nombre $J$ des tours, produits par cette courbe autour de l'axe $O \xi$ est zéro. En même temps l'index. $J$ de rotation est zéro.

Ainsi le théorème de la coincidence de l'index $J$ de la rotation avec zéro peut être regardé pour démontré sur tous les points.

On peut changer la forme de la démonstration de ce théorème de différentes façons. Dans la preuve que nous venons d'énoncer, nous n'avons changé que la dernière des quantités $l_{1}, l_{2}$ et $a$. Mais il est possible de fonder la preuve sur le changement de la quantité $l_{1}$, en ajoutant, s'il devient nécessaire, les ehangements des quantités $l_{2}$ et $a$. Montrons d'une manière brère le plan de cette preure. Avant tout remarquons que les conditions (17) équivalent aux suivantes:

$$
\xi_{0}<1, \quad l_{1}^{2}<g_{0}+\sqrt{\frac{\bar{g}_{2}^{\bar{i}}}{27}}
$$

où

$$
g_{0}=\frac{2 l_{2} B}{3}-\frac{l_{2}^{3} B}{54 \mathrm{e}_{0}^{2}} \text {. }
$$

Admettons que ces conditions soient satisfaites par le système donné des quantités $l_{1}, l_{z}$ et $a$ et admettons que $l_{1}$ diffère de zéro. Puis augmentons continuellement la quantité $l_{1}{ }^{2}$ depuis sa valeur initiale jusqu'à la limite:

$$
g_{0}+\sqrt{\frac{g_{2}^{3}}{27}}
$$

en l'approchant infiniment de ceite limite. Les conditions (17) seront toujours remplies, en même temps la quantité $\Delta=g_{2}{ }^{3}-27 g_{3}{ }^{2}$ décroîtra en approchant infipiment de zéro, les racines $\xi_{2}$ et $\xi_{3}$ de l'équation $R^{2}=0$ se rapprocheront et deviendront égales dans la limite, avec quoi $\xi$ coïncide avec chacune d'elles $\left(\xi=\xi_{\eta}=\xi_{3}\right)$. Ce cas de la limite est examiné en détails dans le $\$ 12$, où il est démontré que d'après la condition:

$$
\xi^{2}<a^{2}\left(1-\xi^{2}\right)
$$

les mouvements asymptotiques existept eł les courbes fermées $S_{-\infty}$ et $S_{+\infty}$ se transforment en points $b^{\prime}$ et $b^{\prime \prime}$. En même temps chacune des courbes fermées, $\Sigma_{-\infty}$ et $\Sigma_{+\infty}$ se transforme aussi en le point correspondant de la sphère $O$ et fait le nombre $J$ de tours autour de l'axe $O \zeta$ égal évidemment à zéro. Cependant si le changement contivn désigné de $l_{1}{ }^{2}$ jusqu'à

$$
g_{0}+\sqrt{\frac{g_{2}^{3}}{27}}
$$

conduit non pas à la condition (126 $6^{\prime}$ mais à la condition inverse, il faut augmenter $a^{2}$ et atteindre par ce moyen la satisfaction de la condition $\left(126^{\prime}\right)$. Si avee cela on rencontrait un obstacle dans ce que la différence $1-\xi^{2}$ serait zéro, on pourrait éloigner cet obstacle en changeant $l_{2}$ de façon que $1-\xi^{2}$ ne fat pas zéro. C'est par des changements continuels 
pareils qu'on obtient toujours l'exécution de la condition $\left(126^{\prime}\right)$, avec laquelle les trajectoires $\Sigma_{-\infty}$ et $\Sigma_{+\infty}$ deviennent des points et font chacune zéro de tours autour de l'axe $O \xi$. Par conséquent l'index $J$ doit être aussi zéro pour ce mouvement ci et pour le mouvement qui correspond aux valeurs initiales des quantitées $l_{1}, l_{2}$ et $a$.

Passons à présent à l'examen des conséquences du théorème de la coïncidence de l'index $J$ avec zéro. Nous concernerons les signes, qui permettent parfois de juger sans calculer la quantité caractéristique $h$, si les mouvements asymptotiques périodiques du point $v$ avec la période $2 \omega$ sont possibles ou non, suivant les circonstances présentes.

Si l'index $J$ est un zéro, nous nous convainquons par les expliquations données dans les paragraphes précédents, que le principe suivant doit avoir lieu.

Si la longitude $\Phi$ du point $V$ (qui représente aussi l'amplitude de la quantité $v=\xi+i \eta$ ) tend, avec la croissance illimitée du temps $\tau$, en cas limite $\grave{a} \pm \infty$, il n'existe pas de rotations asymptotiques périodiques; mais si pendant la croissance illimitée du temps $\tau$ la longitude $\Phi$ dans la limite garde une valeur finie, les mouvements asymptotiques périodiques doivent exister. On sousentend ici le changement continuel de la longitude $\Phi$ da point $V$ depuis la valeur initiale donnée.

Ce principe, exprimé pour la première fois parMr.B.C.Mlodzeievsky sans démonstration complète, peut être compté pour démontré dans toute sa pleinitude, comme une conséquence des formules (82), (95) et (110) et du théorème concernant la coïncidence de lindex $J$ avee zéro. Nous nous baserons sur ce principe pour la déduction des signes ou des règles, désignés plus bas, qui montrent la possibilité ou limpossibilité des mouvements asymptotiques périodiques du point $v$ avec la période $2 \omega$.

En introduisant dans l'expression (14) à la place de $v$ l'expression:

$$
v=\sqrt{1-\xi^{2}} \cdot e^{\Phi_{i}}
$$

et supposant pour brièveté

$$
\operatorname{tg} \Theta=\frac{-R}{l_{1}}, \quad \gamma=\sqrt{2 \overline{B \varrho_{0}}} \sqrt[3]{\frac{2}{B \varrho_{0}}}, \quad \varrho_{1}=\frac{1}{2 \sqrt{2 B \varrho_{0}}}
$$

nous arons

$$
\frac{d(\Phi-\theta)}{d \tau}=a \gamma \sqrt{\xi}-\overline{\xi_{0}}\left\{\frac{l_{1} e_{1}}{a \sqrt{\left(\xi-\xi_{0}\right)^{3}}}-\cos (\Phi-\theta)\right\} .
$$

Remarquons que l'angle $\Theta$, déterminé par la prenière des expressions (127), est une fonction périodique du temps $\tau$, variable toujours entre deux limites finies. D'ailleurs la fonction

$$
f \tau=\frac{l_{1} e_{1}}{a \sqrt{\left(\zeta-\zeta_{0}\right)^{3}}}
$$

ne change pas de signe pendant le changement de $\tau$ et varie 
toujours entre les limites $b_{2}$ et $b_{3}$, correspondantes aux valeurs de cette fonction pour $\tau=0$ et $\tau=\omega$ et déterminées par les expressions:

$$
b_{2}=\frac{l_{1} \varrho_{1}}{a \sqrt{\left(\xi_{2}-\xi_{0}\right)^{3}}} \text { et } b_{3}=\frac{l_{1} \varrho_{1}}{a V\left(\zeta_{3}-\xi_{0}\right)^{3}} \text {. }
$$

Au moyen des inếgalités (20) et (21) nous nous convainquons que

$$
b_{2}{ }^{2}>b_{3}{ }^{2} \text {. }
$$

N'oublions pas cela et supposons, que pendant tous les changements du temps $\tau$ la fonction $f \tau$ ne devient jamais par sa quantité absolue plus petite que 1. Cela se réalise avec les conditions: $b_{3}{ }^{2} \geqq 1$ et dans ce cas la deuxième partie de l'équation (128) conserre toujours le même signe. Par conséquent la quantité absolue de la différence $\Phi-\Theta$ grandit indéfiniment pendant la croissance da temps $\tau$. Cependant l'amplitude $\Phi$ doit tendre à l'infini pendant la croissance illinitée de $\boldsymbol{x}$. Voilà pourquoi il ne peut pas exister de mouvements asynptotiques périodiques, quand $b_{s}^{2} \geqq 1$.

Si nous supposons, qu' arec tous les changements du temps $\tau$ la fonction $f x$, déteruninée par l'expression $(129)$, ne devient jamais plus grande que 1 par sa quantité absolue, ce qui se réalise arec la condition $b_{2}{ }^{2} \leq 1$, on peut se convaincre au moyen de l'équation (128), que la différence $\Phi-\theta$ doit toujours varier entre deux limites finies pendant la croissance illirnitée de $\tau$. Effectivement la croissance continuelle de la quantité $\Phi-\Theta$ doit toujours, suivant ces conditions, venir prendre la valeư de zéro de la deuxième partie de l'équation (128) et ensuite changer son signe, c. à. d. que la croissance de la quantité $\Phi-\Theta$ doit se changer en décroissance; cette décroissance de la quantité $\Phi-\theta$ doit conduire à ce que la dérivée $\Phi-\theta$ vienne prendre la valeur de zéro, puis changer le signe de la deuxième partie de l'équation $(198)_{3}$ c. à. d. que $\Phi-\Theta$ doit commencer à croître, et ainsi de suite. Pour représenter ces variations avec plus de clarté, déterminons l'angle $\Psi$ des conditions

$$
\cos \psi=f x, \quad 0<\psi<\pi,
$$

puis imaginons nous sur la sphère $O$ deux courbes, décrites, pendant le changement de $\tau$ depuis 0 jusqu'à $\omega$, par des points qui représentent deux quantités:

$$
\sqrt{1-\zeta^{2}} \cdot e^{ \pm} \Psi^{i}
$$

Ces courbes présenteront deux transversales $l^{\prime}$ et $l^{\prime \prime}$ de la zone sphérique incernée entre les circonférences $F_{2}$ et $F_{3}$; ces courbes seront disposées symétriquement par rapport au plan $\xi O \xi$. Ces transversales partagent la zone désignée en deux domaines, pour lesquels la seconde partie de l'équation (128) garde l'an ou l'autre signe, selon le domaine dans lequel se trouve le point 3 , qui représente la quantité:

$$
v=\sqrt{1-\xi^{2}} \cdot e^{(\Phi-\theta) i}
$$


la seconde partie de l'équation (128) devient zéro avec la position du point $\mathfrak{B}$ sur l'une ou l'autre des transversales. Pendant la croissance da temps $\tau$ l'amplitude $\phi-\theta$ augmente ou diminue, selon la position du point $\mathfrak{B}$ dans l'un ou dans l'autre des deux domaines désignés. Il est facile de voir en plus que le point $\mathfrak{S}$ avec l'une et l'autre de ces positions se mouve pendant la croissance de $\tau$ dans la direction d'une seule et même transversale que nous désignerons par $l^{\prime}$. Le point $\mathfrak{B}$, représentant la quantité $\mathfrak{b}$, déterminée par l'expression (133), balance près de cette transversale $l^{\prime}$ pendant le temps $\tau$, exprimant de grandes valeurs positives. Effectivement il est facile de se convaincre au moyen de l'équation (128) et de l'expression (133), que si dans le moment donné le point $\mathfrak{B}$ est passé du côte du plan $\xi O \xi$, où se trouve la transversale $l$, ce point ne peat pas traverse de nouveau le plan $\xi O \zeta$ pendant la eroissance de $\tau$ commençant de ce moment. Par conséquent les passages da point $\mathfrak{B}$ par la transversale $l^{\prime \prime}$ sont impossibles à partir de ce moment, ce ne sont que les passages de la transversale $l^{\prime}$ qui sont possibles. Ces passages du point $\mathfrak{B}$ d'une rive de la transversale $l^{\prime}$ à l'autre se repetent infiniment beuucoup de fois. La comparaison des dérivées

$$
\frac{d \Psi}{d \tau} \quad \text { et } \quad \frac{d(\Phi-\Theta)}{d \tau}
$$

montre le suivant. Les intersections de la trajectoire du point $\mathfrak{B}$ avec la transversale $l$ sont telles que les passages du point mobile $\mathfrak{B}$ allant de la première rive de la transversale $l^{\prime}$ à l'autre s'accomplissent dans les phases $\tau+2 m \omega,-\omega<\tau<0$ et les passages inverses dans les phases $\tau+2 m \omega, 0<\tau<\omega$. Le point $\mathfrak{B}$ peat aussi faire une infinité de passages à travers l'autre transversale $l^{\prime \prime}$, mais cela se produira avec la déeroissance de $\tau$ jusqu'à $-\infty$. Avec les mouvements désignés du point $\mathfrak{B}$ l'amplitude $\Phi-\Theta$ de la quantité $\mathfrak{v}$ correspondante, ainsi que l'amplitude $\Phi$ de la quantité $v$, ayant $\tau=+\infty$, restent finies. Par conséquent, quand $b_{2}^{2} \leqq 1$ nous devons avoir deux mouvements asymptotiques périodiques du point $v$ avec la période $2 \omega$.

Ainsi nous pouvons exprimer la règle suivante.

I. Règle, Si $b_{3}{ }^{2} \geq 1$, il n'existe pas de mouvements asymptotiques dériodiques du point $v$ (la quantité caractéristique $h$ doit ètre imaginaire). Si $b_{2}{ }^{2} \leqq 1$, il doit exister des mouvements asymptotiques périodiques du point $v$ avec le periode $2 \omega$ (la quantité caractéristique $h$ doit être réelle).

Cette règle ne résoud pas la question dans le cas où

$$
b_{3}{ }^{2}<1<b_{2}{ }^{2} \text {. }
$$

On peut rapetisser le domaine, où la règle n'est pas réelle, en compliquant un peu les calculs. Introduisons l'angle $\Gamma$ auxiliaire en le désignant ainsi: 


$$
\frac{a r}{d x}=a \gamma \sqrt{\xi-\zeta_{0}}\left\{b_{i}-\frac{l_{1} e_{1}}{a \sqrt{\left(\xi-\xi_{0}\right)^{3}}}\right\}
$$

où $b_{4}$ est une quantité constante. Choisissons la quantité $b_{4}$ à condition, que l'angle $\Gamma$ reste toujours fini pendant la croissance illimitée de $\tau$. Pour avoir les circonstances, qui permettront la réalisation de cette condition, il faut remarquer, que la deuxième partie de l'expression (135) est une fonction périodique paire avec le période $2 \omega$ et par conséquent elle doit se développer suivant les cosinus d'ares multiples en série de la forme:

$$
\frac{d \Gamma}{d \tau}=A_{0}+A_{1} \cos \frac{\pi \tau}{\omega}+A_{2} \cos \frac{2 \pi \tau}{\omega}+\cdots
$$

En même temps l'angle $\Gamma$ sera représenté ainsi:

$$
\Gamma=\text { Const. }+A_{0} \tau+\frac{\omega}{\pi}\left[A_{1} \sin \frac{\pi \tau}{\omega}+\frac{1}{2} A_{2} \sin \frac{z \pi \tau}{\omega}+\cdots\right] .
$$

Cet angle ne restera fini pendant toutes espèces de changements de $\tau$ que dans le cas où

$$
A_{0}=0 \text {. }
$$

Après avoir écrit cette condition sous une forme plus étendue, nous en obtiendrons la quantité $b_{4}$, exprimée ainsi:

$$
b_{1}=\frac{l_{10_{1}} \int_{0}^{\omega} \frac{d \tau}{\xi-\xi_{0}}}{\int_{0}^{\omega} \sqrt{\xi-\zeta_{0}} d \tau} .
$$

Au moyen de cette expression et de l'expression:

$$
\int \sqrt{\zeta-\xi_{i}} d \tau=\sqrt{\left(z-\xi_{0}\right)^{3}} \int_{0}^{\omega} \frac{d \tau}{\xi-\xi_{0}}
$$

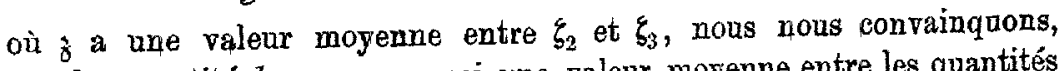
que la quantité $b_{4}$ occupe aussi une valeur moyenne entre les quantités $b_{2}$ et $b_{3}$, c. ̀̀. d. que

$$
b_{3}{ }^{2}<b_{4}{ }^{2}<b_{2}{ }^{2}
$$

Après avoir additionné les expressions (135) et (128) nous trourons:

$$
\frac{d(\Phi-\theta+\Gamma)}{d \tau}=a \gamma \sqrt{\zeta}-\bar{\zeta}_{0}\left\{b_{4}-\cos (\phi-\theta)\right\} \text {. }
$$

Cette équation montre, que la fonction dérivée $\phi-\theta+\Gamma$ garde tonjours son signe ayant $b_{4}^{2} \geqq 1$ et que par conséquent langle $\Phi-\theta+\Gamma$ de même que l'angle $\Phi$ grandissent indéfiniment pendant la croissance du temps $\tau$. Cependant il n'existe pas de monvements asymptotiques périodiques. Ainsi à la place de la règle $I$ nous pouvons exprimer la règle suivante.

II. Règle. Si $b_{4}{ }^{2} \geq 1$, il n'existe pas de mouvements asymptotiques périodìques du point $v$ (la quantité caractéristique $h$ doit être imaginaire). 
Si $b_{2}{ }^{2} \leqq 1$, il doit exister des mowvements asymptotiques périodiques du point $v$ (la quantité caractéristique $h$ doit être reelle).

Cette règle ne résoud pas la question dans le cas où $b_{4}{ }^{2}<1<b_{2}{ }^{2}$, inégalités, qui correspondent moins au large domaine que les inégalités (134), puisque $b_{3}{ }^{2}<b_{4}{ }^{2}$.

Il est évident qu'il est possible de composer une quantité inombrable de signes pour résoudre la question de l'existence ou de la non-existence des mouvements asymptotiques périodiques. Rappelons nous du signe, proposé par Mr. B. C. Mlodzeiersky, signe, qui se distingue par sa simplicité.

Il est à regretter, que tous les signes connus de cette espèce laissent toujours un certain domaine, où ils ne résolvent pas la question. Si l'on parvient à étraicir ce domaine, ce n'est qu'en compliquant le calcul, et ce n'est pas désirable. Il reste à savoir, si ce défaut est la conséquence de conditions accidentelles, ou si elle provient de l'essence du problème.

\section{$\S 10$.}

Correlations, qui existent entre les rẻsolutions de l'équation (42) et leur quantités conjuguées. Formules pour le calcul des coefficients dans le développement des résolntions particulières fondamentales on séries $\tau$.

Bornons nous à examiner les corrélations pour les résolutions particulières fondamentales $w_{1} \tau$ et $w_{2} \tau$, résolutions qui se développent en séries (51). On abtient facilement ensuite au moyen de ces corrélations d'autres qui correspondent à deux résolutions particulières quelconque de l'équation (42), par exemple aux résolutions canoniques $W_{1} \tau$ et $W_{2} \tau$.

La fonction $v$, déterminée par l'expression (41) est présentée au moyen des résolutions particulières fondamentales $w_{1} \tau$, et $w_{2} \tau$ de la façon suivante:

$$
v=P \tau \cdot \frac{w_{1}^{\prime} \tau+c w_{2}^{\prime} \tau}{u_{1} \tau+c w_{2} \tau}
$$

où $P \tau$ est déterminée au moyen de l'expression (66), et la constante $c$ est déterminée d'après la position initiale du point $v$ sur la circonférence $E_{2}$ (au moment $\tau=0$ ). On peut représenter la valeur initiale correspondante de la quantité $v$ ainsi:

$$
v=\sqrt{1-\xi_{2}^{2}} \cdot e^{\mathfrak{b} i}
$$

où $\mathfrak{b}$ est une quantité réelle. En même temps, quand $\tau=0$, l'équation (138) prend la forme:

$$
c=\gamma_{2} e^{b_{b}}
$$


où $\gamma_{2}$ est une quantité réelle, déterminée par l'expression (86'). C'eșt pourquoi la fonction $v$ est exprimée ainsi:

$$
v=P \tau \cdot \frac{w_{1}^{\prime} \tau+\gamma_{2} e^{b i} w_{2}^{\prime} \tau}{w_{1} \tau+\gamma_{2} e^{b i} w_{2} z}
$$

et la fonction conjuguée de la façon suivante

$$
\bar{v}=\overline{P_{\tau}} \cdot \frac{\bar{w}_{1}^{\prime} \tau+\gamma_{2} e^{-b_{i}} \bar{w}_{2}^{\prime} \tau}{\bar{w}_{1} \tau+\gamma_{2} e^{-\overline{b i}} \bar{w}_{2} \tau} .
$$

En multipliant les expressions (140) et (140') et ayant en vue que

$$
\begin{aligned}
& v \bar{v}=1-\xi^{2}=\frac{1}{4} \sqrt[3]{\frac{4}{B^{2} \rho_{0}}} \cdot \frac{\beta^{\prime} T^{2}-e_{0}^{\prime 2}}{e_{0}-\beta^{\prime}}, \\
& P \tau \cdot \overline{Y_{\tau}}=\frac{1}{4 a^{2}} \sqrt[3]{\frac{4}{\bar{B}^{2} e_{0}^{2}}} \cdot \frac{\wp^{2} T^{2}-e_{0}^{\prime}{ }^{2}}{\left(e_{0}-\wp T\right)^{2}},
\end{aligned}
$$

nous trouvons après les simplifications:

$$
\begin{aligned}
& \left(w_{1} \tau+\gamma_{2} e^{G_{i} i} w_{2} \tau\right)\left(\gamma_{2} \bar{w}_{2} \tau+e^{b i} \bar{w}_{1} \tau\right)= \\
& =\frac{\left(w_{1}^{\prime} \tau+\gamma_{2} e^{b i} w_{2}^{\prime} \tau\right)\left(\gamma_{2} \bar{w}_{2}^{\prime} \tau+e^{b i} \bar{w}_{1}^{\prime} \tau\right)}{a^{2}\left(e_{0}-\wp T\right)} .
\end{aligned}
$$

En disposant les deux parties de cette identitée par les degrés de la quantité arbitraire $z=e^{b i}$ et en composant les coefficients avec les mêmes degrés, nous obtenons les corrélations suivantes:

$$
\left\{\begin{aligned}
w_{1}^{\prime} \tau \cdot \bar{w}_{2}^{\prime} \tau & =a^{2}\left(e_{0}-\wp T\right) w_{1} \tau \cdot \bar{w}_{2} \tau, \\
\bar{w}_{1}^{\prime} \tau \cdot w_{2}^{\prime} \tau & =a^{2}\left(e_{0}-\varphi T\right) \bar{w}_{1} \tau \cdot w_{2} \tau, \\
w_{1}^{\prime} \tau \cdot \bar{w}_{1}^{\prime} \tau+\gamma_{2}{ }^{2} w_{2}^{\prime} \tau \cdot \bar{w}_{2}^{\prime} \tau & =a^{2}\left(e_{0}-\rho T\right)\left(w_{1} \tau \cdot \bar{w}_{1} \tau+\gamma_{2}{ }^{2} w_{2} \tau \cdot \bar{w}_{2} \tau\right) .
\end{aligned}\right.
$$

Ces expressions déterminent deux quantités:

$$
w_{1}^{\prime} \tau \cdot \bar{w}_{1}^{\prime} \tau \text { et } \gamma_{2}^{2} w_{2}^{\prime \prime} \tau \cdot \bar{w}_{2}^{\prime} \tau
$$

au mojen des quantités $w_{1} \tau, \bar{w}_{2} \tau, \bar{w}_{1} \tau, w_{2} \tau$ et justement:

$$
\begin{aligned}
w_{1}^{\prime} \tau \cdot \bar{w}_{1}^{\prime} \tau & =\gamma_{2}{ }^{2} a^{2}\left(e_{0}-\wp T\right) \bar{w}_{2} \tau \cdot w_{2} \tau, \\
\gamma_{2}{ }^{2} w_{2}{ }^{\prime} \tau \cdot \bar{w}_{2}^{\prime} \tau & =a^{2}\left(e_{0}-\wp T\right) w_{1} \tau \bar{w}_{1} \tau .
\end{aligned}
$$

Ces deux expressions et la première des expressions (141) rédnisent les trois corrélations (141) à une forme plus simple:

(142) $\left\{\begin{array}{l}w_{1}^{\prime} \tau \cdot \bar{w}_{1} \tau=\gamma_{2}{ }^{2} w_{2}{ }^{\prime} \tau \cdot \bar{w}_{2} \tau, \quad \bar{w}_{1}{ }^{\prime} \tau \cdot w_{1} \tau=\gamma_{2}{ }^{2} \bar{w}_{2}{ }^{\prime} \tau \cdot w_{2} \tau, \\ w_{1}^{\prime} \tau, \bar{w}_{1}^{\prime} \tau=\gamma_{2}^{2} a^{2}\left(e_{0}-\gamma \mathcal{\gamma}\right) w_{2} \tau \cdot w_{2} \tau\end{array}\right.$

En additionnant les deux équations (142), nous trourons:

$$
\frac{d}{d \tau}\left(w_{1} \tau \cdot \bar{w}_{1} \tau-\gamma_{2}{ }^{2} \bar{w}_{2} \tau \cdot w_{2} \tau\right)=0,
$$

d'où

$$
w_{1} \tau \cdot \bar{w}_{1} \tau-\gamma_{2}^{2} w_{2} \tau \cdot \bar{w}_{2} \tau=\text { Const. }
$$


Après avoir déterminé Const avec $\tau=0$, nous obtenons:

$$
w_{1} \tau \cdot \bar{w}_{1} \tau-\gamma_{2}{ }^{2} w_{2} \tau \cdot \bar{w}_{2} \tau=1 \text {. }
$$

En joignant aux corrélations (142) et (143) la corrélation (53') et si nous en déduisons les expressions $w_{1}^{\prime} \tau, w_{2}^{\prime} \tau, \bar{w}_{1}^{\prime} \tau$ et $\bar{w}_{2}^{\prime} \tau$, nous aboutissons seulement à cinq corrélations différentes que nous représentons ainsi:

$$
\left\{\begin{array}{c}
w_{1}^{\prime} \tau=\gamma_{2}^{2} \cdot i \cdot \bar{w}_{2} \tau \cdot \psi_{1} \tau \cdot e^{i \chi \tau}, \quad \bar{w}_{1}^{\prime} \tau=-\gamma_{2}^{2} \cdot i \cdot w_{2} \tau \cdot \psi_{1} \tau \cdot e^{-i \chi^{\tau}}, \\
w_{2}^{\prime} \tau=i \cdot \bar{w}_{1} \tau \cdot \psi_{1} \tau \cdot e^{i} \chi^{\prime}, \quad \bar{w}_{2}^{\prime} \tau=-i w_{1} \tau \cdot \psi_{1} \tau \cdot e^{-i \chi \tau}, \\
w_{1} \tau \cdot \bar{w}_{1} \tau-\gamma_{2}{ }^{2} w_{2} \tau \cdot \bar{w}_{2} \tau=1
\end{array}\right.
$$

où $\chi \tau$ est déterminée par l'expression $\left(5 \Xi^{\prime \prime}\right)$ et

$$
\psi_{1} \tau=\sqrt{\frac{e_{0}-\rho T}{e_{s}-e_{2}}} .
$$

Passons à présent à la formation des formules pour les calculs des coefficients $\alpha_{2}, \alpha_{3}, \ldots, \beta_{2}, \beta_{3}, \ldots$ dans les développements $(51)$ des résolutions fondamentales particulières $w_{1} \tau$ et $w_{2} \tau$ en séries par les degrès $\tau$. Représentons avant tout les développements par les degrès $\tau$ des fonctions suivantes:

$$
\frac{\wp^{\prime} T}{2\left(\wp T-e_{0}\right)}, \quad \frac{i e_{0}^{\prime}}{2\left(\wp T-e_{0}\right)}, \quad\left(\wp T-e_{0}\right)
$$

dont la première est impaire et les deux dernières sont paires. Ces développements doivent avoir la forme

$$
\begin{aligned}
& \frac{k^{\prime} T}{2\left(\wp T-e_{0}\right)}=A_{0} \tau+A_{1} \tau^{3}+\cdots+A_{n} \tau^{2 n+1}+\cdots, \\
& \frac{i e_{0}^{\prime}}{2\left(\xi T-e_{0}\right)}=B_{0}+B_{1} \tau^{2}+\cdots+B_{n} \tau^{2 n}+\cdots \\
& \wp T-e_{0}=C_{0}+C_{1} \tau^{2}+\cdots+C_{n} \tau^{2 n}+\cdots,
\end{aligned}
$$

où les coefficients $A_{0}, A_{1}, \ldots, B_{0}, B_{1}, \ldots, C_{0}, C_{1}, \ldots$ sont des quantités réelles, calculées d'après les formules citées ci-dessous, empruntées à la théorie des fonctions elliptiques.

Il s'ensuit des séries (145) et (146) que

$$
\begin{aligned}
\frac{1}{2}\left(\frac{\beta^{\prime} T-e_{0}}{\rho T-e_{0}}\right) & =A_{0} \tau+A_{1} \tau^{3}+\cdots+A_{n} \tau^{2 n+1} \cdots \\
& +i\left(B_{0}+B_{1} \tau^{2}+\cdots+B_{n} \tau^{2 n}+\cdots\right) .
\end{aligned}
$$

Nous obtiendrons en introduisant cette série et celle de (147) dans l'équation (42), puis en remplaçant dans cette équation la fonction $w$ par l'une ou l'autre des fonctions $w_{1} \tau$ et $w_{2} \tau$, déterminées par les expressions (51), nous obtiendrons, par le moyen des coefficients indéterminés, des équations pour la détermination des quantités

$$
\alpha_{2}, \alpha_{3}, \ldots, \beta_{2}, \beta_{3}, \ldots
$$


Sur un oas de rotation d'un solide antour d'un point fixe.

Ainsi nous nous convainquons que les quantités $\alpha_{2}, \alpha_{3}, \ldots$ se déterminent successivement par les équations suivantes:

$$
\left\{\begin{array}{c}
2(n+1)(2 n+1) \alpha_{2 n+2} \\
=\sum_{k=0}^{k=n}\left\{\left(2 k A_{n-k}-a^{2} C_{n-k}\right) \alpha_{2 k}-(2 k+1) B_{n-k} a_{2 k+1}\right\} \\
2(n+1)(2 n+3) \alpha_{2 n+3}
\end{array}\right.
$$

où l'on doit supposer: $\alpha_{0}=1, \alpha_{1}=0$, en donnant au nombre $n$ les significations $0,1,2, \ldots$ Nous trourons de la mêpe manière que les quantités $\beta_{2}, \beta_{3}, \ldots$ se déterminent succéssivement par les équations

$$
\left\{\begin{array}{c}
2(n+1)(2 n+1) \beta_{2 n+2} \\
=\sum_{k=0}^{k=n}\left\{\left(2 k A_{n-k}-a^{2} C_{n-k}\right) \beta_{2 k}-(2 k+1) B_{n-k} \beta_{2 k+1}\right\} \\
2(n+1)(2 n+3) \beta_{2 n+3} \\
=\sum_{k=1}^{k=n}\left\{\left[(2 k+1) A_{n-k}-a^{2} C_{n-k}\right] \beta_{2 k+1}+2(k+1) B_{n-k} \beta_{z k+2}\right\}
\end{array}\right.
$$

où l'on doit supposer: $\beta_{0}=0, \beta_{1}=1$, en donnant au nombre $n$ les significations $0,1,2, \ldots$.

En différentiant l'expression (148) d'après $\tau$, nous obtiendrons*):

$$
\left\{\begin{array}{c}
\wp T-\wp\left(T+T_{0}\right)=A_{0}+3 A_{1} \tau^{2}+\cdots+(2 n+1) A_{n} \tau^{2 n}+\cdots \\
\cdots+2 i\left(B_{1} \tau+\cdots+n B_{a} \tau^{2 n-1}+\cdots\right)
\end{array}\right.
$$

oì $T_{0}$ est une quantité, déterminée par l'expression (43') on, autrement, par les expressions (43), Il s'ensuit des expressions (151) et (147), que

$$
\left\{\begin{array}{l}
A_{n}=\frac{\rho^{(2 n)}\left(\omega+\omega^{\prime}\right)-\varphi^{(2 n)}\left(\omega+\omega^{0}+T_{0}\right)}{1.2 .3 \ldots(2 n+1)}, \\
B_{n}=\frac{i \omega^{(2 n)}\left(\omega+\omega^{\prime}+T_{0}\right)}{1.2 .3 \ldots 2 n}, \\
C_{n}=\frac{\phi^{(2 n)}\left(\omega+\omega^{\prime}\right)}{1.2 .3 \ldots 2 n}
\end{array}\right.
$$

où $n=1,2,3, \ldots$ Il faut ajoater aox expressions (152) encore les suivantes:

$$
A_{0}=\frac{\left(e_{2}-e_{1}\right)\left(e_{1}-e_{3}\right)}{e_{2}-e_{0}}, \quad B_{0}=\frac{i e_{0}^{*}}{2\left(e_{2}-e_{4}\right)}, \quad O_{0}=e_{2}-e_{0} .
$$

*) Halpben, t. I, p. 137. 
Par le calcul saccessif des dérivées

$$
\wp^{(m)}\left(\omega+\omega^{\prime}\right) \text { et } \wp^{(m)}\left(\omega+\omega^{\prime}+T_{0}\right),
$$

qui font partie des formules (152), on doit, en vue des expressions:

$$
\begin{aligned}
& \varphi\left(\omega+\omega^{\prime}\right)=e_{1}, \varphi^{\prime}\left(\omega+\omega^{\prime}\right)=0, \\
& \wp\left(\omega+\omega^{\prime}+T_{0}\right)=e_{2}+\frac{\left(e_{2}-e_{1}\right)\left(e_{2}-e_{3}\right)}{e_{0}-e_{2}}, \\
& \beta^{\prime}\left(\omega+\omega^{\prime}+T_{0}\right)=\frac{-\left(e_{2}-e_{1}\right)\left(e_{2}-e_{3}\right) e_{0}^{\prime}}{\left(e_{0}-e_{2}^{\prime}\right.},
\end{aligned}
$$

se servir des corrélations:

$$
\begin{gathered}
\wp^{\prime \prime} u=6(\wp u)^{2}-\frac{1}{2} g_{2} \\
\wp^{(n+2)} u=12 \sum_{k=6}^{k=n-1}(n-1)_{k} \xi^{(k)} u \cdot \wp^{(n-k)} u, \quad(n=1,2, \ldots)
\end{gathered}
$$

Dans la dernière corrélation, que l'on obtient par la formule de Leibnitz, adoptée à l'équation:

$$
\wp^{\prime \prime} u=12 \xi u \cdot \gamma^{\prime} u,
$$

l'expression $(n-1)_{k}$ est un coefficient binominal.

$$
\S 11 .
$$

Cas où $l_{1}=0$.

Ce cas appartient au nombre des plus simples. Mr. Hess a déjà montré que la question dans ce cas se résolvait jusqu'à la fin au seul moyen des fonctions elliptiques.

Dans ce cas on obtient facilement l'expression de l'amplitude $\Phi$ de la quantité $v$. Cette amplitude satisfait l'équation:

d'où nous trouvons

$$
\frac{d \Phi}{d \bar{\zeta}}=\frac{a \sin \Phi}{\sqrt{1-\xi^{2}}}
$$

$$
\operatorname{tg} \frac{\Phi}{2}=c \cdot e^{-a \cdot \arccos 5}
$$

où $c$ est une constante, déterminée par la valeur initiale de la quantité $\Phi$. L'angle $\psi$, déterminé par l'expression (40), ne change pas dans ce cas.

Le cas, que nous examinous, par rapport à la question de l'index $J$ a été séparé dans la théorie énoncée ci-devant; car la trajectoire du point $V$ passe dans ce cas au moins par un des pôles de la sphère $O$. Mais dans les autres rapports ce cas peut servir d'explication pour la théorie générale. Afin de rapprocher ce cas de la théorie générale, examinons le en combinaison avec l'équation (42). 
Dans le cas, que nous examinons, $e_{0}^{\prime}=0$ et l'équation (42) admet les deux résolutions particulières saisantes:

$$
\left\{\begin{array}{l}
w_{1} u=e^{-\frac{1}{2} \alpha i \log \left(\xi+i \sqrt{1-5^{2}}\right)} \\
w_{2} u=e^{-\frac{1}{2} a i \log \left(\xi-i \sqrt{1-5^{2}}\right)}
\end{array}\right.
$$

Si d'après la désignation déjà établie nous sousentendons par $\theta$ l'angle de l'axe $O \zeta$ (Fig. 1) avee la direction de la gravité, nous aurons:

$$
\xi=\cos \theta, \sqrt{1-\xi^{2}}=\sin \theta,
$$

et l'expression (154) prend la forme:

$$
w_{1} \tau=e^{+\frac{1}{2} \alpha \theta}, \quad w_{2} \tau=e^{-\frac{1}{2} \alpha \theta} .
$$

Dans la suite nons distinguerons deax cas: 1) quand $\xi_{0}<1$ et 2) quand $-1<\xi_{0}<+1$. En plus nous examinerons deux cas linite: 1) quand $\xi_{\mathfrak{a}}=-1$ et 2) quand $\xi_{0}=+1$.

$$
\text { Cas oil } l_{1}=0, \xi_{0}<-1 \text {. }
$$

Avec ces conditions les racines $\xi_{1}, \xi_{2}$ et $\xi_{3}$ du polynome $R^{2}$, qui sera présenté sous la forme:

$$
R^{2}=2 B \rho_{0}\left(1-\xi^{2}\right)\left(\xi-\xi_{0}\right)
$$

seront:

$$
\xi_{1}=\xi_{0}, \xi_{2}=-1, \xi_{3}=+1
$$

Les rayons des circonférences $E_{2}$ et $E_{3}$ sont des zéros. Avec le changement de $\tau$ depuis 0 jusqu'à $w$ le point $v$ doit d'abord se monvoir sur un côté da plan $\xi O \eta$ depuis le point $O$ jusqu'à la circonférence $E_{0}$, décrite du centre $O$ par le rayon 1 (circonférence que le point $v$ atteint au moment où $\xi=0$ ); puis avec le changement de $\tau$ depuis - jusqu'à $2 \omega$ le point $v$ doit passer de l'autre êté da plan $\xi O \eta$ (voyez le $\$ 4$ ) et doit aller jusqu'au point $a$. Les rayons des circonférences $F_{2}$ et $F_{3}$ sont aussi des zéros, et les sections de la sphère $O$, auxquelles se disposent ces eirconférences, se changent en tangentes de la sphère. Ces tangentes passent par les pôles (points d'intersection de la sphère $O$ arec l'axe $O \xi$ ). Par conséquent le point $V$ de la sphère $O$ passe, pendant le changement de $\tau$ depuis 0 jusqu'à $a$, d'un pôle de la sphère $O$ à l'autre, en passant par l'équateur $E_{0}$ au moment où $\xi=0$.

Quand le temps $\tau$ passe par chacun des moments $2 m \omega$ et $(2 m+1) \omega$, où $m$ est un nombre entier, la quantité sin $\theta$, qui d'après les expressions (155) et (156) se présente ainsi:

$$
\sin \theta=\sqrt{\left(\xi-\zeta_{2}\right)\left(\xi_{3}-\xi\right)}=\frac{R}{\sqrt{2 B_{0}\left(\xi-\xi_{3}\right)}}
$$


est annullée en changeant de signe* $e^{*}$. Avec ces conditions l'angle $\theta$, déterminé par les expressions (155), doit recevoir l'accroissement $2 \pi$, quand le temps $\tau$, augmentant continuellement, reçoit l'accroissement de $2 \omega$. En même temps les quantités $w_{1} \tau$ et $w_{2} \tau$, déterminées par les expressions $\left(155^{\prime}\right)$, doivent satisfaire les conditions

où

$$
\begin{aligned}
& w_{1}(\tau+2 \omega)=s_{1} w_{1} \tau, \\
& w_{2}(\tau+2 \omega)=s_{2} w_{2} \tau,
\end{aligned}
$$

$$
s_{1}=e^{a \pi} \text { et } s_{i 2}=e^{-a \pi} \text {. }
$$

Par conséquent les résolutions, que nous examinons, $w_{1} \tau$ et $w_{2} \tau$ sont elles-mêmes canoniques, et la quantité caractéristique $h$ sera dans le cas donné:

$$
h=\sqrt{\frac{s_{2}}{s_{1}}}=e^{-a \pi} .
$$

Afin que cette quantité satisfasse la condition (62), prenons la quantité $a$ pour une quantité négative (ce qui s'obtient par le choix de la direction approchante de l'axe $O x$ ).

Comme la quantité caractéristique $h$ est réelle et différente de 1 , il doit exister sous les conditions, que nous examinons, des mourements asymptotiques périodiques du premier et du second genre avec la période $2 \omega$; le mouvement impériodique doit tendre à l'un ou à l'autre des mouvements périodiques, quand le temps $\tau$ tend à $\pm \infty$.

Le mouvement impériodique du point $v$ se détermine au moyen de l'expression:

$$
v=\sin \theta \cdot \frac{e^{+\frac{1}{2} a \theta}-c e^{-\frac{1}{2} a \theta}}{e^{+\frac{1}{2} a \theta}+c e^{-\frac{1}{2} a \theta}}
$$

où l'on peut déterminer la constante $e$ par la position du point $v$ au moment, où $\theta=\frac{\pi}{2}$ et par conséquent $\xi=0$; à ce moment le point $v$ doit se trouver sur la circonférence $E_{0}$, en représentant la quantité de la forme: $v=e^{\beta i}$, et par conséquent nous aurons:

$$
c=-i \cdot e^{\frac{1}{2} a \pi} \cdot \operatorname{tg} \frac{1}{2} \beta \text {. }
$$

La trajectoire du point $v$ passe par l'origine $O$, et la trajectoire du point $V$ passe par les deux pôles de la sphère $O$.

Si le temps $\tau$ reçoit un accroissement, égal au nombre entier $m$ des périodes $2 \omega$, le point $v$ se dispose au point $v_{m}$, représentant la quantité

$$
v_{m}=\sin \theta \cdot \frac{e^{+\frac{1}{2} a \theta}-e^{-2 m \pi a} c e^{-\frac{1}{2} a \theta}}{e^{+\frac{1}{2} a \theta}+e^{-2 m \pi a} c e^{-\frac{1}{2} a \theta}} .
$$

*) Cela s'explique par ce que $\xi_{2}$ et $\zeta_{3}$ sont le minimum et le maximum des fonctions $\zeta$ et par conséquent la quantite $R$, proportionelle à $\frac{d \zeta}{d \tau}$, s'annule aux moments désignés en changeant de signe. 
Quant à ce qui concerne les mouvements périodiques du premier et du second genre avec la période $2 \omega$, l'an d'eux se détermine au moyen des expressions:

$$
v=\sin _{*} \theta, \quad \eta=0, \quad \xi=\sin \theta,
$$

et l'autre an moyen des expressions

$$
v=-\sin \theta, \quad \eta=0, \xi=-\sin \theta
$$

Ces denx mourements sont des mowements de rotation autour de l'axe $O \eta$ (Fig. 1) et ils ne different que par la direction des rotations.

Le mouvement impériodique, déterminé au moyen de l'expression (158), tend au mouvement périodique (159) avec la croissance du produit $a . \tau$ jusqu'à $+\infty$ et au monvement périodique (160) avec la décroissance du produit $a . \tau$ jusqu'à $-\infty$,

Remarquons enfin, que le cas donné, caractérisé, entre autre, par l'inégalité $\xi_{0}<-1$ ou autrement par l'inégalité $l_{2}>2 \rho_{02}$ a lieu, quand la constante $l_{2}$ est suffisamment grande, e. à. d. quand le solide a recu une force vine suffisamment grande.

$$
\text { Cas où } l_{1}=0,-1<\xi_{0}<+1 \text {. }
$$

Arec ces conditions les racines $\xi_{1}, \xi_{2}$ et $\xi_{3}$ da polynome $R^{2}$ seront:

$$
\xi_{1}=-1, \quad \xi_{2}=\xi_{0}, \quad \xi_{3}=+1
$$

Le rayon de la circonférence $E_{3}$ devient zéro; mais le rayon de la circonférence $E_{2}$ est différente de zéro. Si $\xi_{2}<0$, le point $v$ se moure, pendant le changement de $x$ depuis 0 jusqu'à $\omega$, d'abord d'un côté du plan $\xi O \eta$ partasut de la circonférence $E_{2}$ jusquầ la circonférence $E_{0}$ (qu'il atteint au moment où $\xi=0$ ) et puis sur lautre côté du plan $\xi O \eta$ partant de la circopférence $E_{0}$ jusqu'au point $O$. Le point correspondant $V$ de la sphère $O$ se mouve pendant le changement de $\tau$ depuis 0 jusqu'à $\omega$, entre la circonférence $F_{2}$ et le pôle inférieur de la sphère $O$, en passant par l'équateur $E_{0}$ au noment où $\xi=0$. Si $\xi_{2}>0$ le caractère du mouvement ue diffère du précédent que par ce que le point $v$ n'accomplit pasị le passage d'un côté da plan $\xi O_{\eta}$ à l'autre, et que le point $V$ de la sphère $O$ ne fait pas de passage par l'équateur $E_{0}$.

Avec le changement du temps $\tau$ depuis $-\infty$ jusqu'à $+\infty$ la quantité $\sin \theta$ qui, d'après les expressions (155) et (161), se présente ainsi:

$$
\sin \theta=\sqrt{\left(\xi-\xi_{1}\right)\left(\xi_{3}-\xi\right)}
$$

s'annule et change de signe aux moments $(2 m+1) \omega$, où $m$ est un nombre entier. A cause de cela, quand le temps $\tau$, augmentant continuellement, reçoit l'accroissement de $2 \omega$, l'angle $\theta$ se transforme en - $\theta$ et les résolutions (155') $w_{1} \tau$ et $v_{2} \tau$ passent mutuellement l'ane dans l'autre, c. à. d. qu'il se forme une substitution linéaire, déterminée par les expressions: 


$$
\left\{\begin{array}{l}
w_{1}(\tau+2 \omega)=w_{2} \dot{\tau}, \\
w_{2}(\tau+2 \omega)=w_{1} \tau .
\end{array}\right.
$$

Ayant cette substitution nous obtenons les résolutions canoniques:

$$
\left\{\begin{array}{l}
W_{1} \tau=\frac{1}{2}\left(e^{+\frac{1}{2} a \theta}+e^{-\frac{1}{2} a \theta}\right)=\operatorname{Cos}\left(\frac{a \theta}{2}\right), \\
W_{2} \tau=\frac{1}{2}\left(e^{+\frac{1}{2} a \theta}-e^{-\frac{1}{2} a \theta}\right)=\operatorname{Sin}\left(\frac{a \theta}{2}\right),
\end{array}\right.
$$

où $\operatorname{Sin} z$ et $\operatorname{Cos} z$ sont le sinus et le cosinus hyperbotiques de l'argument $z$. Ces résolutions canoniques satisfont les conditions:

$$
W_{1}(\tau+2 \omega)=s_{1} W_{1} \tau, \quad W_{2}(\tau+2 \omega)=s_{2} W_{2} \tau,
$$

où $s_{1}=+1$ et $s_{2}=-1$. Par conséquent la quantité caractéristique $h$ sera imaginaire et s'exprimera ainsi:

$$
h=i=e^{\frac{\pi i}{2}} .
$$

C'est pourquoi il n'existe pas dans le cas présent de mouvements asymptotiques périodiques du premier et du second genre avec la période $2 \omega$. Comme l'amplitude $\varphi$ de la quantité caractéristique $h$, égale à $\frac{\pi}{2}$, est commensurable avec $\pi$, le mouvement du point $v$, arec n'importe laquelle de ses positions initiales sur la circonférence $E_{2}$, sera périodique avec la période $4 \omega$. Ce mouvement se détermine au moyen de l'expression:

$$
v=\sin \theta \cdot \frac{\operatorname{Cos}\left(\frac{a \theta}{2}\right)+c \cdot \operatorname{Sin}\left(\frac{a \theta}{2}\right)}{\operatorname{Sin}\left(\frac{a \theta}{2}\right)+c \cdot \operatorname{Cos}\left(\frac{a \theta}{2}\right)} .
$$

Ici $c$ est une quantité constante, déterminée par la position initiale du point $v$ sur la circonférence $E_{2}$, et elle a la forme: $c=e^{\beta i}$ où $\beta$ est une quantité réelle. La trajectoire du point $V$ passe dans ce cas par le pôle inférieur de la sphère $O$.

Quand $\beta=0$, nous arons: $c=1$ et par conséquent $v=\sin \theta$. Quand $\beta=x$, nous avons: $c=-1$ et $v=-\sin \theta$. Dans ces deux cas le solide se balance autour de l'axe $O \eta$, qui reste immobile.

Si le temps $\tau$ reçoit un accroissement égal au nombre entier $m$ des périodes $2 \omega$, le point $v$, qui représente la quantité (164), se transporte au point $v_{m}$, représentant la quantité

$$
v_{m}=\sin \theta \cdot \frac{\operatorname{Cos}\left(\frac{a \theta}{2}\right)+(-1)^{m} c \cdot \operatorname{Sin}\left(\frac{a \theta}{2}\right)}{\operatorname{Sin}\left(\frac{a \theta}{2}\right)+(-1)^{m} c \cdot \operatorname{Cos}\left(\frac{a \theta}{2}\right)}
$$


Si $m$ est une quantité paire; $v_{m}=v$, mais $m$ est impaire, $v_{m}=v_{1}$, Le cas, caractérisé par les conditions: $l_{1}=0,-1<\varepsilon_{0}<+1$, a lieu quand le solide a regu wne force vive pas trop grande $\left(l_{2}<2 \varphi_{0}\right)$. Entre autre ce mouvement se produit dans le cas, où l'on a fait partir le solide examiné de n'importe quelle position sans vitesses initiales.

$$
\text { Cas ois } l_{1}=0, \xi_{0}=-1 \text {. }
$$

Dans ce cas

$$
R^{2}=2 B \varrho_{0}(1-\xi)\left(1+\xi i^{2}\right.
$$

Les racines $\xi_{1}, \xi_{2}$ et $\xi_{3}$ se présentent ainsi:

$$
\xi_{1}=\xi_{2}=\xi_{0}=-1, \xi_{3}=+1 \text {. }
$$

La seconde des conditions (17) est remplacée par la condition de la limite $\Delta=0$. Le semipériode a change en $\infty, c$ à. d. que la fonction $\xi$ n'a pas de périude réalle dans le cas que nous examinons.

En intégrant l'équation (10) nous trourons:

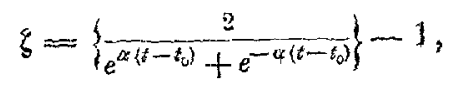

où $t_{0}$ est uine quantité constante et

$$
\alpha=\sqrt{\frac{e_{0}}{B}}
$$

La position du point $v$ sexa déterminée dans ce cas au moyen de l'amplitude $\Phi$, que lon trouve par l'expression (153).

$$
\text { Cas où } l_{1}=0, \xi_{0}=+1 \text {. }
$$

Dans ce cas linite

$$
R^{2}=2 B \varrho_{6}(1-\xi)\left(1-\xi^{2}\right)
$$

Les racines $\xi_{1}, \xi_{2}, \zeta_{3}$ sont représentées à ce moment de la manière suivante:

$$
\xi_{1}=-1, \quad \xi_{2}=\xi_{3}=\xi_{0}=+1 \text {. }
$$

Les conditions (17) se remplacent par les conditions de la limite: $\xi_{0}=1$ et $\Delta=0$. La fonction $\xi$ change en constante égale ì 1 . Le module de la quantité $v$, égal à $\sqrt{1-\xi^{2}}$, est toujours zéro. Le solide tourne également autour de l'axe $O \xi$, qui coincide avec la ligne verticale $O P$. 


\section{$\S 12$.}

Cas où les racines $\xi_{2}$ et $\xi_{3}$ sont égales.

Ce cas limite qui est un cas singulier, sur lequel Mr. N. E. Jouk o vsky a arrêté son attention, appartient aussi au nombre des plus simples. Ce cas est caractérisé par les conditions:

$$
\xi_{0}<1, \Delta=0
$$

remplaçant les conditions (17), qui ont servi de base aux déductions précédentes.

Sous les conditions (167) les racines $\xi_{2}$ et $\xi_{3}$ deviennent égales, La quantité $\xi$, qui, en général, doit varier entre $\xi_{2}$ et $\xi_{3}$, devient dans ce cas particulier la constante:

$$
\left.\xi=\xi_{2}^{*}\right) \text {. }
$$

Les circonférences $E_{2}$ et $E_{3}$ coïncident dans la même circonférence $E$, par laquelle le point $v$ doit se mouvoir. La droite $O \xi$ doit se mouvoir par la surface d'un cone à base circulaire, dont l'axe coïncide avec la ligne verticale $O V$ (Fig. 1). L'équation (14) prend la forme:

$$
\frac{d v}{d t}+\frac{l_{1} i(a v-2 \xi) v}{2 B\left(1-\xi^{2}\right)}+\frac{a l_{1} i}{2 B}=0 .
$$

L'équation (169) peut être intégrée immédiatement, par la séparation des variables. En sousentendant par $b^{\prime}$ et $b^{\prime \prime}$ les racines des équations

$$
a v^{2}-2 \xi v+a\left(1-\xi^{2}\right)=0,
$$

résolue par rapport à $v$, et supposant

$$
\gamma=\frac{a l_{1}\left(b^{\prime}-b^{\prime \prime}\right)}{2 B\left(1-\xi^{\prime \prime}\right)}
$$

nous trouvons:

$$
v=\frac{b^{\prime \prime}-b^{\prime} c e^{i \gamma t}}{1-c e^{i \gamma^{t}}}
$$

où $c$ est une constante, déterminée par la position initiale du point $v$ sur la circonférence $E$.

Le caractère da mouvement que nous examinons dépend de celui des racines $b^{\prime}$ et $b^{\prime \prime}$. Ainsi il nous faut distinguer ici trois cas:

*) L'équation (10) est satisfaite par des solutions singulières $\xi=\xi_{2}$ et $\xi=\xi_{3}$ même avec $\Delta>0$. Mais ces résolutions particulières, quand $\Delta>0$, n'amènent pas la solution du problème concernant le mouvement, car avec ces solutions quelques unes des équations (2) ne peuvent pas être satisfaites. 
1) quand les racines $b^{\prime}$ et $b^{\prime \prime}$ sont des imaginaires canjuguées, 2) quand les racines $b^{\prime}$ et $b^{\prime \prime}$ sont réelles et inégales et 3) quand les racines $b^{\prime}$ et $b^{\prime \prime}$ sont réelles et égales. Le troisième cas est un cas intermédiaire par rapport aux deux premiers.

Premier oas. Supposons que

$$
\zeta^{2}<a^{2}\left(1-\xi^{2}\right)
$$

c. à. d. que les racines $b^{\prime}$ et $b^{\prime \prime}$ de l'équation (170) sont imaginaires et inégales. La quantité $\gamma$, déterminée par l'expression (171), est aussi dans ce cas une imaginaire de la forme:

$$
\gamma=i \gamma_{1}
$$

où $\gamma_{1}$ est une quantité réelle, que nous compterons aussi comme positive (ce qui s'obtient par le choix du signe du radical dans les expressions des racines $b^{\prime}$ et $b^{\prime \prime}$ ). Avec ces conditions et arec l'exigence de placer le point $v$ sur la circonférence $E$, l'expression (162) prendra la forme:

$$
v=b^{\prime \prime} \cdot \frac{1-b^{\prime} z e^{-\gamma_{1} t}}{1-b^{\prime \prime} z e^{-\gamma_{1} t}}
$$

où $z$ est une quantité réelle arbitraire constante.

En examinant l'expression (174) daus deux cas particuliers: 1) quand $z=0$ et 2) quand $z=\infty$, nous nous convainquons que dans ces cas le solide se mouve de façon à ce que le point $v$ reste en repos, occupant soit la position $b^{\prime \prime}$ (si $z=0$ ), soit la position $b^{\prime}$ (si $z=\infty$ ). Par conséqueut le solide doit tourner dans chacun de ces deux cas autour de l'axe $O V$ [avec une vitesse constante, comme le montre l'expression (40)]. Les positions immobiles $b^{\prime}$ et $b^{\prime \prime}$ da point $v$ seront nommées pans ces deux cas positions asymptotiques du premiex et du second genre. Ces positions du point $v$ sont symétriques par rapport à l'axe $O \xi$, puisque les quantités $b^{\prime}$ et $b^{\prime \prime}$ sont conjuguées.

Examinons ensuite l'équation (174), admettant que la quantité $z$ soit finie et différente de zéro. Dans ce cas le point $v$ se déplace continuellement sur l'un des deux ares de la circonférence $E$, partagée en deux parties par les points $b^{\prime}$ et $b^{\prime \prime}$, et s'approche sans limite ou de la position asymptotique $b^{\prime \prime}$ (si $t$ augmente jusqu'à $+\infty$ ), ou de la position asymptotique $b^{\prime}$ (si $t$ diminue jusqu'à $-\infty$ ).

Il est évident que le cas, que nous examinons, présente un cas limite (quand $\xi_{3}-\xi_{2}$ tend à zéro) du mouvement, exploré dans le $\S 6$. Aux deux mouvements asymptotiques périodiques désignés du point $v$ correspondent en cas limite les deux positions asymtotiques $b^{\prime}$ et $b^{\prime \prime}$ du point $v$, c. à. d. les trajectoires fermées $S_{t^{-\infty}}$ et $S_{-\infty}$ dans la limite (quand $\xi_{3}-\xi_{2}$ tend à zéro) se transforment d'une manière correspondante en 
points $b^{\prime \prime}$ et $b^{\prime}$. En même temps les courbes $\Sigma_{-\infty}$ et $\Sigma_{+\infty}$ changent aussi en points; ainsi on voit immédiatement que le nombre $J$ des tours, produits par chacune de ces courbes autour de l'axe $O \xi$, doit être zéro. Cette remarque de même que la constance de l'index $J$ avec le changement de $l_{1}, l_{2}$ et $a$ a servi plus haut $(\$ 9)$ de base à une des preuves du théorème qui démontre que $J=0$.

La quantité caractéristique $h$ (désignée plus bas) doit être réelle dans ce cas.

Second cas. Supposons que

$$
\xi^{2}>a^{2}\left(1-\xi^{2}\right),
$$

c. à. d. que les racines $b^{\prime}$ et $b^{\prime \prime}$ de l'équation (170) sont réelles inégales. An moyen de cette condition et avee l'exigence que le point $v$ reste sur la circonférence $E$, l'expression (172) se présentera ainsi:

$$
v=\sqrt{1-\zeta^{2}} \cdot \frac{b^{\prime \prime}+\sqrt{1-\xi^{2}} \cdot e^{i(\gamma t+\vartheta)}}{\sqrt{1-\xi^{2}}+b^{\prime \prime} \cdot e^{i(\gamma t+\vartheta)}}
$$

où $\vartheta$ est une quantité constante arbitraire réelle.

Avec le changement du temps $t$ le point $v$ se déplace sur la circonférence $E$ sans tendre à aucune position asymptotique et en décrivant la circonférence entière $E$ dans la période de temps représenté par la quantité*)

$$
\frac{2 \pi}{\gamma}
$$

Il est évident que ce mouvement présente un cas limite (quand $\xi_{3}-\xi_{2}$ tend à zéro) du mouvement qui a été examiné dans le $\$ 7$. La quantité caractéristique $h$ (désignée plus bas) doit être imaginaire dans ce cas.

Troisième cas. Supposons que

$$
\xi^{2}=a^{2}\left(1-\xi^{2}\right)
$$

c. à. d. que les racines $b^{\prime}$ et $b^{\prime \prime}$ de l'équation (170) sont égales $\left(b^{\prime}=b^{\prime \prime}=\frac{\xi}{a}\right)$. Dans ce cas l'intégrale de l'équation (169) peut être exprimé ainsi:

$$
v=\frac{\xi}{a}\left\{1+\frac{2 \gamma^{\prime}}{i(t+z)-\gamma^{\prime}}\right\},
$$

où

$$
\gamma^{\prime}=\frac{B \xi}{a^{2} l_{1}},
$$

*) Cette période a une valeur singulière qui n'appartient qu'au cas limite que nous examinons. Cette période existe même quand la quantité caractéristique $h$, étaut imaginaire, a l'amplitude $\varphi$ incommensurable avee $\pi$. 
et $z$ est une constante arbitraire, à laquelle on doit donner une valeur réelle pour que le point $v$ puisse se poser sur la circonférence $E_{\text {, }}$

Quand $z= \pm \infty$, l'expression (178) se réduit à la forme:

$$
v=\frac{\xi}{a}=b_{0} \text {. }
$$

Dans ce cas le solide se moure de façon que le point $v$ reste toujours en repos en occupant une position $b_{0}$ que nous nommerons asymptotique. Le solide tourne dans ce cas régulièrement antour de la verticale $O V$.

Supposons maintenant que la quantité $z$ de l'équation (178) soit finie. Dans ce cas le point $v$ se déplace, pendant le changement continuel du temps $t$, sans interruption sur la circonférence $E_{x}$ en approchant sans limite de la position asymptotique $b_{i},\left(178^{\prime}\right)$ quand le temps $t$ croât jusqu'à $+\infty$ ou quand il dếcroît jusqu'à $-\infty$.

Il est évident que le monvement que nous examinons présente le cas limite du mouvement qui a été examiné dans le $\$ 8$.

\section{Remarque.}

Dans le cas que nous examinons la demi-période co, déterminée par l'expression $(29)$, se présente, pour le variable $F$, de cette façion:

$$
\omega=\frac{1}{2} \int_{e_{1}}^{\infty} \frac{d y}{\left(y-e_{2}\right) \sqrt{y-e_{1}}}=\frac{\pi}{2 \sqrt{e_{1}-e_{2}}} .
$$

Si nous passons au moyen de la deuxième des expressions (23) au variable $t$, la demi-période $\Omega$ s'exprimera pour ce variable de la façon suivante:

$$
\Omega=\frac{B \pi}{2 \sqrt{\varepsilon_{1}-e_{k}}} \sqrt[3]{\frac{a}{B \rho_{0}}}=\pi \sqrt{\frac{B \xi}{\hat{Q}_{0}\left(1+3 \xi^{2}\right)}} .
$$

La quantité caractéristique $h$ se présentera [comme il est facile de le voir ạ moyen de l'expression (172)] comme l'une des quatre quantités:

$$
\pm e^{ \pm i r s}
$$

où $\gamma$ est déterminé par l'expression (171) et

$$
\gamma \Omega=\pi \sqrt{\frac{\xi^{2}-a^{2}\left(1-\xi^{2}\right)}{1+3 \xi^{2}}} .
$$

Sous les copditions (173) la quantité $h$ sera représertée ainsi:

$$
h=e^{Y_{2} \Omega} \text {, }
$$

par quoi la condition (62) sera remplie.

Sous la condition (175) l'amplitude $\varphi$ de la quantité caractéristique $h$ sera: 
530 P. A. Nekrassofe. Sur un cas de rotation d'un solide autour d'un point fixe.

(182)

$$
\varphi=\gamma \Omega,
$$

par quoi, comme le montre l'expression (180), les conditions (63) seront satisfaites. Donc

$$
h=e^{i \gamma \Omega} .
$$

Nous avons consideré dans les $\$ \$ 6-8$ les courbes fermées de la forme $\sigma_{m}$. Dans le cas limite quand $\zeta_{2}=\zeta_{3}$, la courbe fermée $\sigma_{m}$ représente l'arc $D_{m} D_{m+1}$ passé deux fois en sens inverses. Le nombre $J$ des tours, produits autour de l'axe $O \xi$ par la courbe $\sigma_{m}$, est évidemment égai à zéro. Ainsi nous trouvons la démonstration nouvelle du théorème sur l'index $J$. 